\title{
Polycomb paralog chromodomain inhibitors active against both CBX6 and CBX8
}

\author{
Natalia Milosevich, ${ }^{[a],[+]}$ Chelsea R. Wilson, ${ }^{[a],[t]}$ Tyler M. Brown, ${ }^{[a]}$ Aktan Alpsoy, ${ }^{[b]}$ Sijie Wang, ${ }^{[b]}$ Katelyn \\ E. Connelly, ${ }^{[b]}$ Kirsten A. D. Sinclair ${ }^{[a]}$ Felino R. Ponio, ${ }^{[a]}$ Rebecca Hof, ${ }^{[a]}$ Emily C. Dykhuizen, ${ }^{[b]}$ and \\ Fraser Hof ${ }^{*}[a]$
}

In memory of François Diederich: enthusiastic supporter, motivator, and mentor
[a] N. Milosevich, C.R. Wilson, T.M. Brown, K.A.D Sinclair, F.R. Ponio, R. Hof, Prof. F. Hof Department of Chemistry
University of Victoria
Victoria, BC, V8W 3V6, Canada
fhof@uvic.ca
[b] A. Alpsoy, S. Wang, K.E. Connelly, Prof. E.C. Dykhuizen
Department of Medicinal Chemistry and Molecular Pharmacology
College of Pharmacy, Purdue University and Purdue University Center for Cancer Research
575 Stadium Mall Drive, West Lafayette, Indiana 47906, United States
[†] Equal Contribution

Supporting information for this article is given via a link at the end of the document

\begin{abstract}
Methyllysine reader proteins bind to methylated lysine residues and alter gene transcription by changing the compaction state of chromatin or by the recruitment of other multiprotein complexes. The polycomb paralog family of methyllysine readers bind to trimethylated lysine on the tail of histone 3 via a highly conserved aromatic cage located in their chromodomains. Each of the polycomb paralogs are implicated in several disease states. CBX6 and CBX8 are members of the polycomb paralog family with two structurally similar chromodomains. By exploring the structure-activity relationships of a previously reported $\mathrm{CBX} 6$ inhibitor we have discovered more potent and cell permeable analogs. Our current report includes potent, dual-selective inhibitors of CBX6 and CBX8. We have shown that the -2 position in our scaffold is an important residue for selectivity amongst the polycomb paralogs. Preliminary cell-based studies show that the new inhibitors impact cell proliferation in a rhabdoid tumor cell line.
\end{abstract}

\section{Introduction}

Post-translational modifications (PTMs) can be used as epigenetic signatures that control and regulate gene expression pathways. Lysine methylation is one such PTM that is important in several malignancies. ${ }^{[1]}$ Lysine methylation on histones, which package and order DNA, can activate or repress transcription of the associated genes. Methyllysine 'reader' proteins that recognize this mark form multi-protein complexes that regulate fundamental pathways critical for health and development. ${ }^{[2]}$

Chromodomains (chromatin organization modifier domains) are a family of methyllysine readers that include proteins belonging to the Heterochromatin protein-1 (HP1) and polycomb sub-families. Both families recognize higher methylation states on $\mathrm{H} 3 \mathrm{~K} 9$ and $\mathrm{H} 3 \mathrm{~K} 27 .{ }^{[3]}$ The polycomb family of methyllysine readers consists of five human paralogs: $\mathrm{CBX} 2, \mathrm{CBX} 4, \mathrm{CBX} 6, \mathrm{CBX} 7$, and $\mathrm{CBX} 8$. The human $\mathrm{CBX}$ polycombs are important regulatory proteins that are responsible for cellular differentiation during development, stem cell maintenance via transcriptional repression, and cancer progression. ${ }^{[4]}$ Each member can participate in different versions of the multi-protein complex called polycomb repressive complex 1 (PRC1). Each is functionally unique $^{[4 b, 5]}$, and while the biological data are growing rapidly the phenotypes of chemical inhibition are only recently beginning to be discovered.

The challenge in targeting the polycomb paralogs with selective chemical inhibitors is due to the high degree of sequence similarity within the chromodomains. The polycomb paralog chromodomains all possess a conserved aromatic cage made up of 3-4 aromatic residues that recognize the side chain of Kme3. They achieve selectivity by recognizing residues adjacent to the $\mathrm{Kme} 3$ in a " $\beta$-groove" that engages the Kme3-containing partner in a beta-sheet like structure. The main differences between polycomb chromodomains are relatively small variations in the side chains that line the $\beta$-groove.

CBX7-selective inhibitors are by now well known, ${ }^{[6]}$ but other CBX's are also arising as promising new drug targets. CBX6 and CBX8 are both involved in regulating stem cell differentiation. CBX6 is an essential regulator of embryonic stem cells (ESCs). ${ }^{[7]}$ Depletion of CBX6 in ESCs leads to rapid cell differentiation. Cellbased studies with CBX6 mutants lacking the two methyllysinebinding tryptophan residues in the aromatic cage showed that the function of CBX6 in ESCs is strongly linked to the methyllysine reading function of the chromodomain. ${ }^{[7]}$ Unlike CBX7 (and apparently all other human polycomb paralogs), CBX6 does not act through binding H3K27me3. ${ }^{[3,8]}$ Ablation of the H3K27 methyldepositing PRC2 complex in ESCs did not change CBX6 recruitment to chromatin. $\mathrm{CBX} 8$ plays a critical role in dictating cell fate decisions and activation of differentiation genes. ${ }^{\left[{ }^{[]}\right.} \mathrm{CBX} 8$ acts as part of the PRC1 complex and is upregulated during ESC differentiation. CBX7 in ESCs acts to maintain pluripotency and is downregulated upon increased expression of CBX8 during differentiation. $\left.{ }^{[4 b, 5 b}, 9\right]$

Both CBX6 and CBX8 have arisen as potential targets in oncology. Overexpression of CBX6 promotes tumor growth and is linked to poor patient outcomes in hepatocellular carcinoma (HCC). ${ }^{[10]}$ In vitro studies in multiple HCC cell lines showed increased CBX6 expression to promote cell growth whereas knockdown of CBX6 showed reductions in proliferation. In vivo studies also showed $\mathrm{CBX} 6$ overexpression to promote $\mathrm{HCC}$ tumour growth. ${ }^{[10]} \mathrm{CBX} 6$ is also upregulated in rhabdoid tumours, an aggressive pediatric cancer. ${ }^{[11]}$ The oncogenic role of CBX8 has been demonstrated in several malignancies including hepatocellular carcinoma $(\mathrm{HCC}),{ }^{[12]}$ acute myeloid leukemia $(\mathrm{AML}),{ }^{[13]}$ breast cancer ${ }^{[14]}$ and esophageal carcinomas. ${ }^{[15]} \mathrm{CBX} 8$ is also overexpressed in bladder cancer, ${ }^{[16]}$ choriocarcinomas, ${ }^{[17]}$ and glioblastoma multiforme. ${ }^{[18]}$ 
Isolated examples of selective inhibition of CBX6 and CBX8 have recently been reported. A peptidic CBX6 inhibitor (3) developed by structure-based design achieved selectivity over the other polycomb paralogs due to installation of a bulky group directed into protein's $(-2)$ pocket, which is larger in CBX6 than other polycombs. ${ }^{[19]}$ In separate work, a DNA-encoded library showed that CBX8-selective inhibition was achievable by combining large groups in the $(-2)$ pocket with other changes in the ligand structure along the $\beta$-groove. ${ }^{[20]}$ Similarly a CBX8selective positive allosteric modulator was found by incorporating larger groups in the $(-2)$ pocket along with other structural changes. ${ }^{[21]}$

In this work, we thoroughly define the structure-activity relationships (SAR) of CBX6 inhibition, and also report a second generation of CBX6 inhibitors with improved cell permeability. As we modified ligands in the $\beta$-groove region, we arrived at a novel class of dual-active CBX6- and CBX8-inhibiting ligands and identified the structural features that give rise to this dual selectivity. We provide preliminary biological data that demonstrate that the inhibitors have functional effects in a cancer cell line.

\section{Results}

We started our efforts with modifications to previously reported CBX6-targeting peptidic inhibitors, ${ }^{[19,22]}$ and chose to screen each compound against $\mathrm{CBX} 1 / 6 / 7 / 8$. This panel was chosen in order to efficiently obtain data on CBX6- and CBX8-selectivity over CBX7, which is the $C B X$ that intrinsically binds ligands most strongly. It also includes CBX1 (HP1 $\beta$ ) as a representative of the HP1 class of chromodomains. Our previous report on CBX6 inhibition included the initial scaffold 1, the promiscuous inhibitor 2, CBX6selective inhibitor 3 that is $6-90$-fold selective for CBX6 over CBX1, 7 and 8, 4 the dye-free analog of 2; and compound 5 the dye-free analog of 3 (Figure 1). ${ }^{[19,22]}$ As a first step towards making lower molecular weight compounds, we investigated $\mathbf{1}$, which is an analog of $\mathbf{2}$ that lacks the C-terminal Glu residue and dye label (Figure 1, Table 1). Compound 1 is 10 -fold selective for CBX7 over CBX1 and 6- and 29-fold selective over CBX6 and CBX8, respectively.

To improve the cell-permeability and drug-like properties of our inhibitors we first focused on understanding the roles of the dye and the $(-2)$ substituent in $\mathrm{CBX} 6 / 8$ selectivity (Table 1). Substituting the alanine in compound $\mathbf{4}$ to a valine in compound $\mathbf{5}$ produced a $>100$-fold decrease in binding to CBX7. Removal of the FITC-labeled lysine on the scaffold containing the $(-2)$ valine residue (2 compared to 4 ) resulted in a 20-, 40- and 3-fold decrease in binding to CBX6, CBX7 and CBX8, respectively. ${ }^{[19,22]}$ We knew from previous reports that removal of the FITC dye label makes these compounds significantly less potent. From the current experiments we can also see that the FITC removal affects CBX6 and CBX8 binding differently. Although $\mathbf{5}$ is still selective for CBX6 over CBX7, it is no longer selective for CBX6 over CBX8. Compound 5 is equipotent against $\mathrm{CBX} 6 / 8\left(\mathrm{IC}_{50}\right.$ values of $\sim 17 \mu \mathrm{M}$ ) and is 12 -fold selective over CBX7. Compound 6 , in which an isoleucine residue is present at the $(-2)$ position, displayed no measurable binding to CBX1 or CBX7 $(>500 \mu \mathrm{M})$. Compound 6 displayed similar potency for CBX6 and CBX8 $\left(\mathrm{IC}_{50}\right.$ values of 9 and $14 \mu \mathrm{M})$.

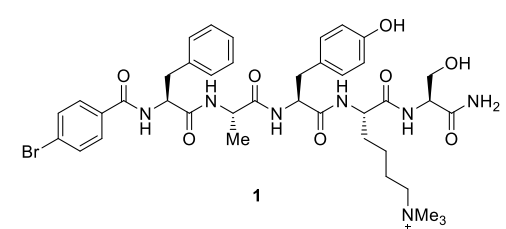

(1)

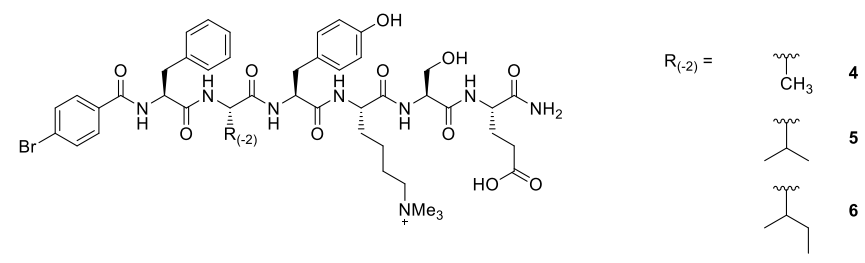

Figure 1. Chemical structures of compounds 1-6.

Table 1. $I C_{50}$ values for compounds $1,4,5$ and $6(\mu \mathrm{M}) .{ }^{[a]}$

\begin{tabular}{ccccc}
\hline Compound & CBX1 & CBX6 & CBX7 & CBX8 \\
\hline $\mathbf{1}$ & $14^{[\mathrm{b}]}$ & $8^{[\mathrm{b}]}$ & $1.4^{[\mathrm{b}]}$ & $41^{[\mathrm{b}]}$ \\
$\mathbf{4}$ & N.T. $^{[\mathrm{c}]}$ & 5 & 1.8 & 28 \\
$\mathbf{5}$ & N.T. $^{[\mathrm{c}]}$ & 17 & $210^{[\mathrm{b}]}$ & $18^{[\mathrm{b}]}$ \\
$\mathbf{6}$ & $>500^{[\mathrm{b}]}$ & 9 & $>500$ & 14 \\
\hline
\end{tabular}

[a] Competitive FP assays performed in triplicate unless stated otherwise. Raw data and asymmetric 95\% confidence intervals are reported in SI.

[b] Competitive FP assays performed in duplicate

[c] Not tested

We focused next on optimization of compound 5 by removing the quaternary ammonium ion (Kme3) and the Glu residue, which we hypothesized would each cause poor cell permeability. We removed the $\mathrm{C}$-terminal glutamic acid residue to reduce overall charge, leading to compound 7 , and made an analogous compound 8 that swapped trimethyllysine (Kme3) for diethyllysine (Ket2) (Figure 2). ${ }^{[6]}$ Removal of the C-terminal Glu gave decreased binding to $\mathrm{CBX} 7$ and no significant change in potency was observed for CBX6/8 (Table 2). Replacement of Kme3 with Ket2 gave a 7-fold increase in binding to CBX6, and no change in binding to CBX8. Compound 8 is 6 -fold selective for CBX6 over CBX8 and displays no binding to $C B \times 1 / 7$. We next sought to substitute the $(-1)$ residue in $\mathbf{8}$ with a more hydrophobic leucine residue (9, Figure 3 ). Compound 9 showed a slight decrease in potency for CBX6 and a 4-fold increase in binding to CBX8 (Table 3). Neither compound showed activity against CBX1 or CBX7. 
<smiles>CC(C)C(NC(=O)C(Cc1ccccc1)NC(=O)C(Cc1ccc(O)cc1)NC(=O)[C@H](CO)NC(=O)c1ccc(Br)cc1)C(=O)NC(CO)C(N)=O</smiles><smiles>CCNC(=O)C(CCCCN(C)CC)NC(=O)C(CO)NC(=O)C(Cc1ccc(O)cc1)NC(=O)[C@H](NC(=O)[C@H](Cc1ccccc1)NC(=O)c1ccc(Br)cc1)C(C)C</smiles>

Figure 2. Structure of compounds 7 and Ket2 analog 8

Table 2. Binding affinity for compounds 7 and 8 ( $\left(\mathrm{C}_{50}\right.$ values in $\left.\mu \mathrm{M}\right)$. [a]

\begin{tabular}{ccccc}
\hline Compound & CBX1 & CBX6 & CBX7 & CBX8 \\
\hline 7 & $>500$ & 24 & $>250$ & 18 \\
8 & $>500$ & 3.2 & $>500$ & 18 \\
\hline
\end{tabular}

[a] Competitive FP assays performed in triplicate unless stated otherwise. Raw data and asymmetric $95 \%$ confidence intervals are reported in SI.

We next explored the roles of $\mathrm{N}$-terminal capping ( $\mathrm{N}$-cap) residues on $\mathrm{CBX6/8}$ selectivity, following leads from our collaborative studies of CBX8-selective inhibitors generated by the Krusemark lab. ${ }^{[20]}$ Using the scaffold with leucine at the $(-1)$ position (9), we substituted the $\mathrm{N}$-cap residue with two distinct heterocycles. Substitution of the $p$-bromobenzamide group with $1 \mathrm{H}$-pyrazole-3-carboxamide $(10)$ resulted in a 10 -fold decrease in binding to CBX6 and a 15-fold decrease in binding to CBX8 reminiscent of results previously seen with a DEL (Table 3). ${ }^{[20 a]}$ Substitution with the 5-methylisoxazole-3-carboxamide (11) gave a similar binding profile as 9 with CBX6/8 and showed activity against CBX7 with an $\mathrm{IC}_{50}$ value of $105 \mu \mathrm{M}$ (Table 3). A similar profile was observed for a 5-methylisoxazole $\mathrm{N}$-cap ligand with a tyrosine residue at the $(-1)$ position (12).

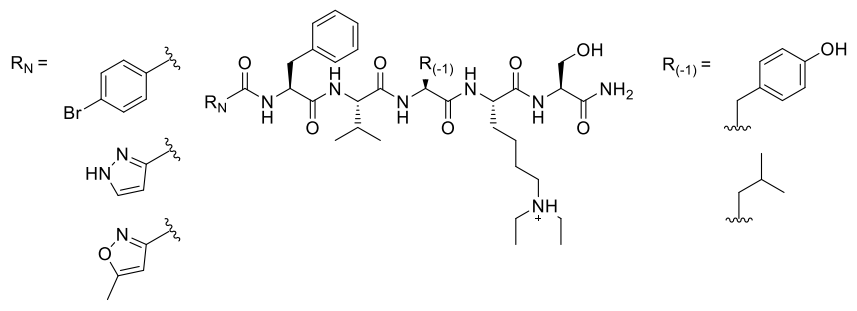

Figure 3. Chemical structures of compounds 8-12 with $(-1)$ and N-cap substitutions.

Table 3. Binding affinity for compounds 8-12 (IC 50 values in $\mu \mathrm{M})$. ${ }^{[a]}$

\begin{tabular}{lllllll}
\hline $\mathrm{R}(\mathrm{N})$ & $\mathrm{CBX} 1$ & $\mathrm{CBX} 6$ & $\mathrm{CBX7}$ & $\mathrm{CBX} 8$ \\
\hline 9 & $>300$ & 3.2 & $>500$ & 18 \\
\end{tabular}

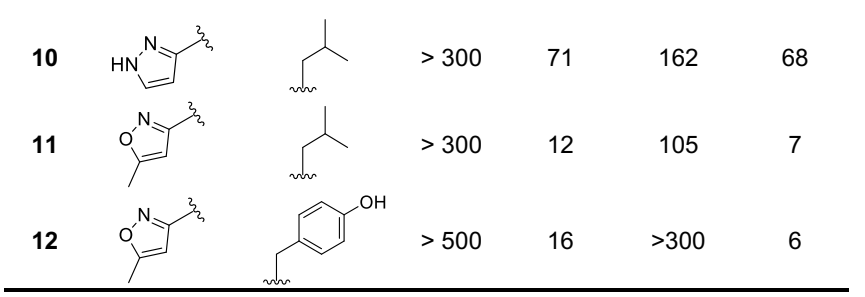

[a] Competitive FP assays performed in triplicate unless stated otherwise. Raw data and asymmetric $95 \%$ confidence intervals are reported in SI.

[b] Competitive FP assays performed in duplicate

To explore the SAR in the $\beta$-groove we combined the $\mathrm{N}$-cap heterocyclic substitutions with aromatic and hydrophobic rings in the $(-3)$ position (Figure 4, Table 4). Leucine was held constant as the $(-1)$ residue. Replacement of the phenylalanine residue in 10 with a cyclohexyl group (13) showed a slight 2-3-fold increase in binding to CBX6, 7 and 8 . The same substitution in the 5methylisoxazole-3-carboxamide containing 11 to give compound 14 did not significantly change binding to CBX6 and CBX8 but did show a decrease in binding to CBX7 (Table 4).

Modifying the aromatic residues at the $(-3)$ position while holding the $\mathrm{N}$-cap methylisoxazole constant showed a decrease in binding to CBX7. Replacement of the phenyl moiety (11) with a 4-methoxyphenyl group (15) resulted in no significant change in binding to CBX6. CBX7 binding was decrease 2-fold while CBX8 was increase 12-fold. Substituting the methoxy for fluorine (16) and iodine (17) abolished binding to CBX7. The p-fluorophenyl (16) analog showed no significant change in CBX6 and 3-fold decrease in CBX8 binding, while the $p$-iodophenyl (17) showed a 6- and 33- fold decrease in binding to CBX6 and CBX8. Exchanging the phenyl moiety (11) with a pyridine-3-yl group at the $(-3)$ position decreased binding to CBX6/7/8 by a factor of 3 , 4 and 2 respectively (18, Table 4$)$. Replacing the pyridine-3-yl with a thiophene (19) showed a 7-, 2- and 10- fold increase to CBX6/7/8 binding.

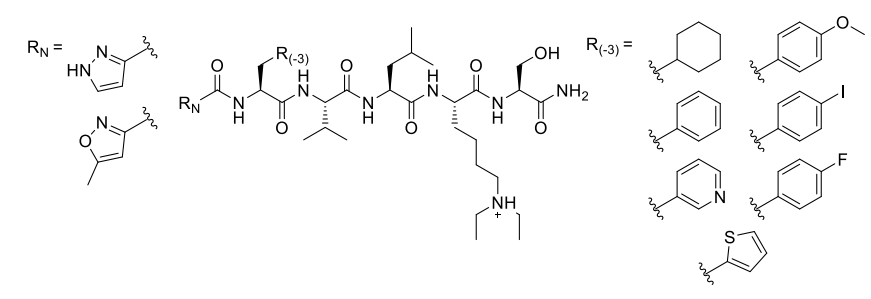

Figure 4. Overall chemical structures of compounds 10, 11 and 13-19 with (-3) and $\mathrm{N}$-cap substitutions. See Table 4 for compound numbers

Table 4. Binding affinity for compounds 10,11 and 13-19 $\left(\mathrm{IC}_{50}\right.$ values in $\left.\mu \mathrm{M}\right){ }^{[a]}$

\begin{tabular}{|c|c|c|c|c|c|c|}
\hline & $R(N)$ & $R(-3)$ & CBX1 & CBX6 & CBX7 & CBX8 \\
\hline 10 & & & $>300$ & 71 & 162 & 68 \\
\hline 13 & & & $>300$ & 32 & 104 & 25 \\
\hline 11 & & & $>300$ & 12 & 105 & 7 \\
\hline 14 & & & $>300$ & 8 & 171 & 6 \\
\hline 15 & & & $>500$ & 12 & 175 & $0.6^{[b]}$ \\
\hline 16 & & & N.T. $\left.{ }^{[c]}\right]$ & 9.3 & $>500$ & 1.8 \\
\hline
\end{tabular}




N.T. [c] 68 >500

[a] Competitive FP assays performed in triplicate unless stated otherwise. Raw data and asymmetric 95\% confidence intervals are reported in SI.

[b] Estimated value because $\mathrm{IC}_{50}$ is outside of linear range of assay. ${ }^{[22-23]}$ [c] Not tested

Substitution of the methylisoxazole $\mathrm{N}$-cap gave large improvements in potency. We synthesized compounds $\mathbf{2 0}$ and $\mathbf{2 1}$, containing a propyl substitution in the 5-position of the isoxazole ring (Figure 5, Table 5). The 5-propylisoxazole $\mathrm{N}$-cap combined with the $(-3)$ cyclohexyl moiety (20) displayed potent binding to CBX6 with an $\mathrm{IC}_{50}$ value of $2.4 \mu \mathrm{M}$ and is 17 - and 3-fold selectivity over CBX7 and CBX8. 5-propylisoxazole ( $\left.R_{N}\right)$ with a phenylalanine $(-3)$ and threonine $(-1)$ lead to a potent CBX8 inhibitor (21) with an $\mathrm{IC}_{50}$ of $2.2 \mu \mathrm{M}$. This inhibitor was 3- and 120fold selective for $\mathrm{CBX} 8$ over $\mathrm{CBX} 6$ and $\mathrm{CBX7}$. The 5phenylisoxazole $\mathrm{N}$-cap substitution in compounds $\mathbf{2 2}$ and $\mathbf{2 3}$ lead to the most potent inhibitors. The addition of a 5-phenylisoxazole $\mathrm{N}$-cap substitution with a $(-3)$ phenyl group provided $\mathbf{2 2}$, which is the most potent compound of the series (Table 5). Compound 22 is equipotent for CBX6 and CBX8 with $I_{50}$ values of $200 \mathrm{nM}$ and is 18-fold selective over CBX7. We then swapped the $(-1)$ leucine for a tyrosine to obtain compound 23. Compound $\mathbf{2 3}$ was one of the more potent compounds however it had no significant selectivity amongst $C B \times 6 / 7 / 8$. Substitution of the methyl group in the 5-methylisoxazole $\mathrm{N}$-cap with larger alkyl chains and aromatic groups improves potency due to increased engagement with the extended $\beta$-groove of CBX6/7/8.
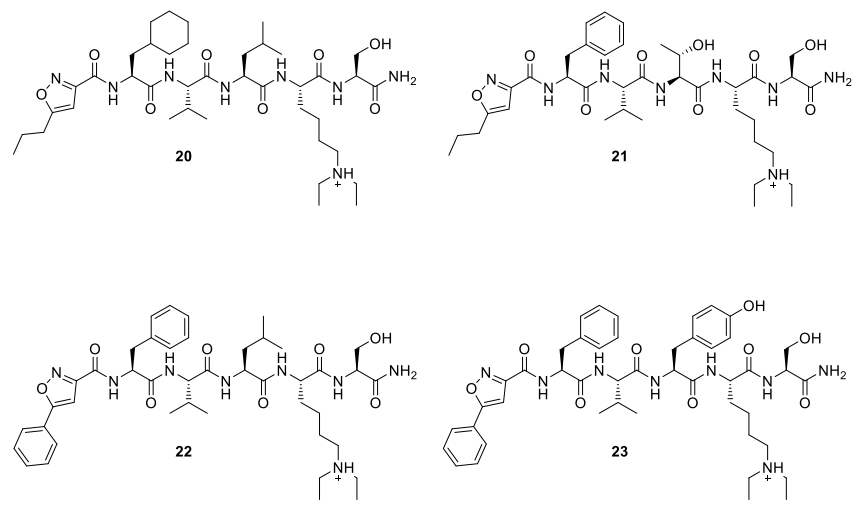

Figure 5. Chemical structure of peptidic ligands 20-23 with isoxazoles, (-1) and (-3) substitutions.

Table 5. Binding affinity for compounds 20-23. (IC 50 values in $\mu \mathrm{M})$. [a]

\begin{tabular}{ccccc}
\hline Compound & CBX1 & CBX6 & CBX7 & CBX8 \\
\hline $\mathbf{2 0}$ & $>300$ & 2.4 & 40 & 7.4 \\
$\mathbf{2 1}$ & N.T. ${ }^{[\mathrm{b}]}$ & 7.5 & 268 & 2.2 \\
$\mathbf{2 2}$ & $>500$ & 0.2 & 3.6 & $0.2^{[\mathrm{c}]}$ \\
$\mathbf{2 3}$ & N.T. ${ }^{[\mathrm{b}]}$ & 2.2 & 5.4 & 1.0 \\
\hline
\end{tabular}

[a] Competitive FP assays performed in triplicate unless stated otherwise. Raw data and asymmetric $95 \%$ confidence intervals are reported in SI.

[b] Not tested [c] Estimated value because $\mathrm{IC}_{50}$ is outside of linear range of assay. ${ }^{[22-23]}$

Larger hydrophobic substitutions in the $(-2)$ position of the ligand allow for CBX8 selective inhibitors. We synthesized analogs of compound $\mathbf{1 1}$ containing a cyclopropyl, cyclopentyl and sec-butyl group at the $(-2)$ position (Figure 6). Compound 24 containing a cyclopropyl group was nearly equipotent for CBX6/8 and displayed between 6- and 7-fold selectivity over CBX7 (Table 6). Compared to the isopropyl-containing compound (11), the cyclopropyl substitution increased binding to CBX6/7/8 by factors of 2-5. Replacing the cyclopropyl with a cyclopentyl group (25) dramatically reduced binding to $\mathrm{CB} \times 6 / 7$ and decreased binding to CBX8 by a factor of 6 ( $\mathrm{IC}_{50}$ of $18 \mu \mathrm{M}$, Table 6$)$. Compound 25 is $10 \mathrm{x}$ and 20x selective for CBX8 over CBX6 and CBX7 respectively. Addition of a sec-butyl group (26) further decreased binding to $\mathrm{CBX6}$ and abolished binding to $\mathrm{CBX7}$ at the concentrations tested. All CBX polycomb paralogs tested showed decreased binding with increasing size of the substituent at the (2) position.

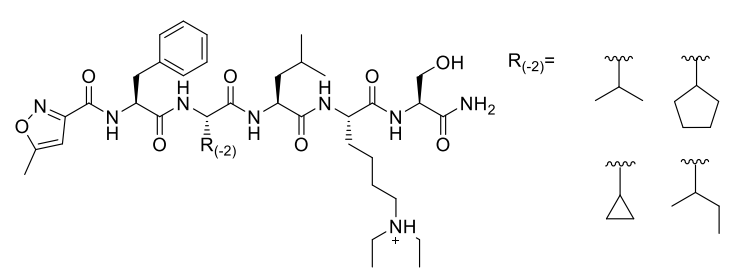

Figure 6. Chemical structure of peptidic ligands 11, 24-26 with substitutions at the $(-2)$ position.

Table 6. Binding affinity for compounds $11,24-26$. ( $\left(\mathrm{C}_{50}\right.$ values in $\left.\mu \mathrm{M}\right)$. ${ }^{[a]}$

\begin{tabular}{cccccc}
\hline Compound & $\mathrm{R}(-2)$ & $\mathrm{CBX} 1$ & $\mathrm{CBX} 6$ & $\mathrm{CBX7}$ & $\mathrm{CBX8}$ \\
\hline 11 & $>300$ & 12 & 105 & 7 \\
24 & $>500$ & 3.6 & 22 & 3.0 \\
25 & $>500$ & 178 & 372 & 18 \\
\hline
\end{tabular}

[a] Competitive FP assays performed in triplicate unless stated otherwise. Raw data and asymmetric $95 \%$ confidence intervals are reported in SI.

CBX8 was best able to tolerate larger substitutions directed into the $(-2)$ pocket of the protein. We and others have reported CBX8-selective ligands that engage the large size $(-2)$ pocket within $\mathrm{CBX} 8$ compared to other members of the polycomb paralog CBX family. ${ }^{[20-21]}$ The SAR reported demonstrates the $(-2)$ pocket of CBX8 to be capable of engaging and binding to ligands with bulkier $(-2)$ substitutions. The residues lining the $(-2)$ pocket are nearly identical in CBX6/8 with the exception of an isoleucine residue in CBX6 that is replaced with a leucine in CBX8. Compound 25 , containing a cyclopentyl group at the $(-2)$ position displays promising selectivity for CBX8. We predict that 
combining the $(-2)$ cyclopentyl group with other substitutions could improve the potency and selectivity of $\mathbf{2 5}$ for CBX8.

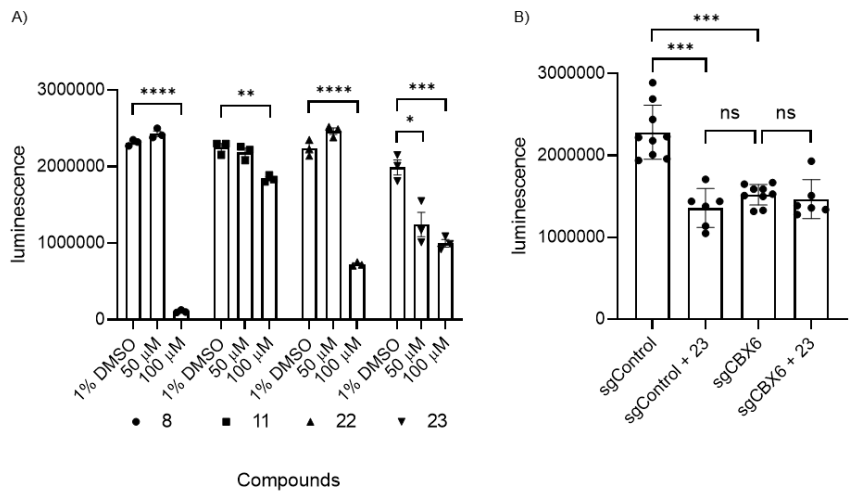

Figure 7. Inhibitors are cell permeable and able to engage CBX proteins. A) G401 rhabdoid cells are affected by treatment of 8, 11, 22, 23 and show decreased cell proliferation. Cell counts were analyzed using CellTitre-Glo following 72-hour treatment. B) Treatment with compound 23 with wild type G401 decreased cell proliferation while no effect was observed in CBX6 KO. P value calculated using students t-test, $n=6$. ${ }^{* *} p<.001$

CBX6 is upregulated in rhabdoid tumours, ${ }^{[11]}$ a highly aggressive pediatric malignancy driven by loss of SMARCB1 subunit of the SWI/SNF chromatin remodeling complex. ${ }^{[11,24]}$ We used immunoblot analysis to profile CBX6 expression in a panel of kidney cell lines, including non-transformed epithelial cells (HK2), clear cell renal cancers (Caki1, Caki2, 786O), kidney adrenal caner (SW-13) and kidney rhabdoid tumor cell line G401, as well as muscle rhabdoid cell line A204. There was no detectable CBX6 protein in the majority of the cell lines, while the kidney rhabdoid line G401 displayed a high expression level of CBX6, consistent with patient transcriptional data (Figure S47a). We used CRISPRCas9 to knock out CBX6 in the G401 cell line (Figure S47b) and determined that loss of $\mathrm{CBX} 6$ significantly reduces the growth rate (Figure S47c). To investigate whether CBX chromodomain inhibition can recapitulate the effects of CBX6 knockout in rhabdoid cells, we measured cell proliferation of G401 rhabdoid cells following treatment with 8, 11, 22 and 23. Wild-type G401 cells were treated with the inhibitors for 72 hours and cell counts were measured using CellTitre-Glo (Figure 7a). While all four inhibitors displayed significant toxicity at $100 \mu \mathrm{M}$, only compound 23 reduced growth to a similar degree as CBX6 knockout and was active at $50 \mu \mathrm{M}$. To define whether compound 23 is targeting CBX6 function, we treated wild-type and CBX6 KO G401 cells (Figure $7 \mathrm{~b}$ ) with $50 \mu \mathrm{M}$ compound 23. Compound $\mathbf{2 3}$ decreased cell proliferation in wild-type but does not affect proliferation in $\mathrm{KO}$ cells, and this provide indirect evidence that it is targeting CBX6. However, inhibition of other CBX chromodomains, particularly with $100 \mu \mathrm{M}$ treatment, cannot be excluded. Further experiments are needed to determine whether $\mathrm{CBX} 7$ and $\mathrm{CBX} 8$ play a role in rhabdoid tumor viability, and whether these ligands might also be inhibiting them.

\section{Discussion}

These studies show the critical role the $(-2)$ position of CBX ligands for selectivity within the polycomb paralog family. The ability of ligands with larger groups in the $(-2)$ position to bind the CBX proteins depends on the peptide scaffold and neighboring residues. Previous work demonstrated dye-labeled ligands with a valine residue in the $(-2)$ position to be selective for CBX6. Removal of the dye from these scaffolds showed reduced selectivity for CBX6 over CBX8, however CBX6 was still better able to tolerate ligands containing bulkier groups within the $(-2)$ pocket (compound 3, 6, Table 1). Larger groups in the $(-2)$ position within the scaffolds reported demonstrate selectivity for CBX8. The aromatic cage, hydrophobic clasp and (-2) pocket are tightly connected and residues in the ligand to the right and left of the Ket2 can change the shape and size of the $(-2)$ pocket. Despite the highly similar sequence homology of the residues in the $(-2)$ pocket in $\mathrm{CBX} 6 / 8$, our results show a clear difference in the binding effects within this pocket depending on the chemical structure of the ligand and resulting protein-ligand interactions.

Aromatic and hydrophobic residues are well tolerated at the (3 ) position of the ligand. The greatest selectivity for CBX8 within the $(-3)$ series was achieved through the addition of a 4-methoxy phenyl moiety. Further exploration of substituted aromatic rings in the $(-3)$ position of the ligand will aid in a better understanding of selectivity determinants.

The 5-phenylisoxazole $\mathrm{N}$-cap moiety in compound $\mathbf{2 2}$ was the most potent dye-free $\mathrm{CBX} 6 / 8$ inhibitor in our series of compounds. Replacement of the 5-methyl to the 5-phenyl group on the terminal isoxazole ring improved binding to CBX6 and 8 by a factor of 60 and 35 . These results clearly demonstrate the potential for potent inhibitors to be developed through extension of the ligand to engage the proteins' peptide binding $\beta$-groove.

Developing cell-active chromodomain inhibitors is challenging and there are limited examples that have achieved this goal. In spite of the significant interest in CBX's as drug targets, very few phenotypes for CBX inhibition have been reported. Of the 15 reports of chromodomain inhibitors, ${ }^{[6,19-22,25]}$ there are 5 examples of cell-active CBX inhibitors. $\left.{ }^{[6 a}, 66,6 \mathrm{~h}, 20 \mathrm{~b}, 21\right]$ The small molecule inhibitor MS37452 (Kd $30 \mu \mathrm{M})$ was shown to be active in cells but required compound treatment of $>250 \mu \mathrm{M}$ to observe decreased amounts of CBX7 on a known target locus. ${ }^{[6]}$ The aforementioned UNC3866, with approximately $5 \%$ cell permeability, was the first example of a cell-active peptidic chromodomain inhibitor ${ }^{[6]]}$ The use of DNA-encoded libraries to target the CBX proteins resulted in the discovery of a CBX8-selective and cell-permeable peptidic inhibitor. ${ }^{[20 b]}$ In addition, the first positive allosteric modulator of CBX7 was reported and shown to regulate PRC1 activity in cellular assays. ${ }^{[6 h]}$ A second allosteric modulator was recently published for CBX8 that showed activity in two different cancer cell lines. ${ }^{[21]}$ The cell-based studies reported demonstrate that our second generation inhibitors are able to enter cells and engage CBX proteins. Further studies will be required to determine the observed effects are due only to engagement of CBX6 or also through $\mathrm{CBX} 7 / \mathrm{CBX} 8$, and to what extent they are inhibited in a cellular context.

\section{Conclusion}

CBX6 and CBX8 are emerging therapeutic targets for multiple aggressive malignancies. We have reported the first dualselective inhibitors for CBX6 and CBX8, and demonstrated their entry into cells. These are among the first examples of inhibitors that bind specific CBX polycomb paralogs with no measurable activity against CBX7. The inhibitors and SAR described in this work will pave the way for future efforts in chromodomain inhibition. Cell permeable chemical probes will aid in unraveling the mysteries of CBX6 and elucidate the paralog-specific roles of both $\mathrm{CBX} 6$ and $\mathrm{CBX} 8$.

\section{Experimental Section}

All experimental procedures along with compound characterizations are reported in the Supporting Information. 


\section{Funding}

Canadian Cancer Society Research Institute (Innovation Grant 703789); Prostate Cancer Foundation of British Columbia (Pioneer Award to N.M.), NIH (U01CA207532).

\section{Acknowledgements}

F.H. thanks the Canada Research Chairs program for ongoing support.; N.M thanks the West Coast Motorcycle Ride to Live and CIHR for scholarship support. We thank Casey Krusemark and James McFarlene for helpful discussions. We thank Michael Gignac and Alok Shaurya for technical assistance.

\section{Abbreviations}

CBX, chromobox; DEL, DNA-encoded library; DNA, deoxyribonucleic acid; ESC, embryonic stem cell; FITC, fluorescein isothiocyanate; FP, fluorescence polarization; Glu, glutamic acid; $\mathrm{H} 3$, histone 3; H3K27, lysine 27 on histone $3 ; \mathrm{H} 3 \mathrm{~K} 9$, lysine 9 on histone 3 ; $\mathrm{H} 3 \mathrm{~K} 9 \mathrm{me} 3$, trimethylated lysine 9 on histone 3; H3K27me3, trimethylated lysine 27 on histone 3; $\mathrm{HCC}$, hepatocellular carcinoma; HP1, heterochromatin protein; IC $\mathrm{C}_{50}$, inhibitory concentration that reduces effect by 50 percent; Ket2, diethyllysine; Kme3, tri-methyllysine; KO, knockout; N.T., not tested; PRC1, polycomb repressive complex 1; PRC2, polycomb repressive complex 2; PTM, post-translational modification; SAR, structure activity relationship.

Keywords: Methyllysine reader proteins polycomb paralogs • $\mathrm{CBX}$ inhibitors $\cdot \mathrm{CBX} 6 \cdot \mathrm{CBX} 8$

a) Z. Chen, L. Wang, Q. Wang, W. Li, Epigenomics 2010 2, 551-560; b) E. L. Greer, Y. Shi, Nat. Rev. Genet. 2012 13, 343-357; c) R. Hamamoto, V. Saloura, Y. Nakamura Nat Rev Cancer 2015, 15, 110-124.

[2] a) N. Milosevich, F. Hof, Biochemistry 2016, 55, 1570-1583; b) T. Wagner, D. Robaa, W. Sippl, M. Jung, ChemMedChem 2014, 9, 466-483.

[3] L. Kaustov, H. Ouyang, M. Amaya, A. Lemak, N. Nady, S. Duan, G. A. Wasney, Z. Li, M. Vedadi, M. Schapira, J. Min, C. H. Arrowsmith, The Journal of biological chemistry 2011, 286, 521-529.

[4] a) A. P. Bracken, K. Helin, Nat. Rev. Cancer 2009, 9, 773784; b) L. Morey, G. Pascual, L. Cozzuto, G. Roma, A. Wutz, S. A. Benitah, L. Di Croce, Cell Stem Cell 2012, 10 47-62; c) H. Richly, L. Aloia, L. Di Croce, Cell. Death Dis. 2011, 2, e204.

[5] a) K. Klauke, V. Radulovic, M. Broekhuis, E. Weersing, E. Zwart, S. Olthof, M. Ritsema, S. Bruggeman, X. Wu, K. Helin, L. Bystrykh, G. de Haan, Nat Cell Biol 2013, 15, 353362; b) A. O'Loghlen, A. M. Munoz-Cabello, A. GasparMaia, H. A. Wu, A. Banito, N. Kunowska, T. Racek, H. N. Pemberton, P. Beolchi, F. Lavial, O. Masui, M. Vermeulen, T. Carroll, J. Graumann, E. Heard, N. Dillon, V. Azuara, A. P. Snijders, G. Peters, E. Bernstein, J. Gil, Cell Stem Cell 2012, 10, 33-46; c) H. L. Chan, L. Morey, Trends Biochem Sci 2019, 44, 688-700.

[6] a) C. Ren, K. Morohashi, A. N. Plotnikov, J. Jakoncic, S. G. Smith, J. Li, L. Zeng, Y. Rodriguez, V. Stojanoff, M. Walsh, M. M. Zhou, Chem. Biol. 2015, 22, 161-168; b) C. Ren, S. G. Smith, K. L. Yap, S. Li, J. Li, M. Mezei, A. S. Vincek, F. Aguilo, M. J. Walsh, M.-M. Zhou, ACS Med. Chem. Lett. 2016, 7, 601-605; c) C. Simhadri, K. D. Daze, S. F. Douglas, N. Milosevich, L. Monjas, A. Dev, T. M. Brown, A.
K. H. Hirsch, J. E. Wulff, F. Hof, ChemMedChem 2019, 14, 1444-1456; d) C. Simhadri, K. D. Daze, S. F. Douglas, T. T. Quon, A. Dev, M. C. Gignac, F. Peng, M. Heller, M. J Boulanger, J. E. Wulff, F. Hof, J Med Chem 2014, 57, 2874 2883; e) C. Simhadri, M. C. Gignac, C. J. Anderson, N. Milosevich, A. Dheri, N. Prashar, R. T. Flemmer, A. Dev, T. G. Henderson, S. F. Douglas, J. E. Wulff, F. Hof, ACS Omega 2016, 1, 541-551; f) J. I. Stuckey, B. M. Dickson, N. Cheng, Y. Liu, J. L. Norris, S. H. Cholensky, W. Tempel, S. Qin, K. G. Huber, C. Sagum, K. Black, F. Li, X.-P. Huang, B. L. Roth, B. M. Baughman, G. Senisterra, S. G. Pattenden, M. Vedadi, P. J. Brown, M. T. Bedford, J. Min, C. H. Arrowsmith, L. I. James, S. V. Frye, Nat. Chem. Biol. 2016, 12, 180-187; g) J. I. Stuckey, C. Simpson, J. L. Norris-Drouin, S. H. Cholensky, J. Lee, R. Pasca, N. Cheng, B. M. Dickson, K. H. Pearce, S. V. Frye, L. I. James, Journal of Medicinal Chemistry 2016, 59, 8913-8923; h) K N. Lamb, D. Bsteh, S. N. Dishman, H. F. Moussa, H. Fan, J. I. Stuckey, J. L. Norris, S. H. Cholensky, D. Li, J. Wang, C. Sagum, B. Z. Stanton, M. T. Bedford, K. H. Pearce, T. P Kenakin, D. B. Kireev, G. G. Wang, L. I. James, O. Bell, S. V. Frye, Cell chemical biology 2019, 26, 1365-1379.e1322. A. Santanach, E. Blanco, H. Jiang, K. R. Molloy, M. Sansó, J. LaCava, L. Morey, L. Di Croce, Nature Communications 2017, 8, 1235.

[8] E. Bernstein, E. M. Duncan, O. Masui, J. Gil, E. Heard, C. D. Allis, Mol Cell Biol 2006, 26, 2560-2569.

[9] C. Creppe, A. Palau, R. Malinverni, V. Valero, M. Buschbeck, PLOS Genetics 2014, 10, e1004851.

[10] H. Zheng, W.-h. Jiang, T. Tian, H.-s. Tan, Y. Chen, G.-I. Qiao, J. Han, S.-y. Huang, Y. Yang, S. Li, Z.-g. Wang, R. Gao, H. Ren, H. Xing, J.-s. Ni, L.-H. Wang, L.-j. Ma, W.-p. Zhou, Oncotarget 2017, 8, 18872-18884.

[11] S. Gadd, S. T. Sredni, C. C. Huang, E. J. Perlman, G. Renal Tumor Committee of the Children's Oncology, Lab Invest 2010, 90, 724-738.

[12] a) C. Z. Zhang, S.-L. Chen, C.-H. Wang, Y.-F. He, X. Yang, D. Xie, J.-P. Yun, Cancer Research 2017 canres.0700.2017; b) B. Tang, Y. Tian, Y. Liao, Z. Li, S. Yu, H. Su, F. Zhong, G. Yuan, Y. Wang, H. Yu, S. Tomlinson, X. Qu, S. He, Cell Death \& Disease 2019, 10, 52.

[13] J. Tan, M. Jones, H. Koseki, M. Nakayama, A. G. Muntean, I. Maillard, J. L. Hess, Cancer Cell 2011, 20, 563-575.

[14] C.-Y. Chung, Z. Sun, G. Mullokandov, A. Bosch, Z. A Qadeer, E. Cihan, Z. Rapp, R. Parsons, J. A. AguirreGhiso, E. F. Farias, B. D. Brown, A. Gaspar-Maia, E. Bernstein, Cell Reports 2016, 16, 472-486.

[15] W. Xiao, C. Ou, J. Qin, F. Xing, Y. Sun, Z. Li, J. Qiu, International journal of clinical and experimental pathology 2014, 7, 4817-4826.

[16] G. J. Yuan, X. Chen, J. Lu, Z. H. Feng, S. L. Chen, R. X Chen, W. S. Wei, F. J. Zhou, D. Xie, Cancer Sci 2017, 108 2166-2175.

[17] J. B. Mello, P. D. Ramos Cirilo, O. C. Michelin, M. A Custodio Domingues, M. V. Cunha Rudge, S. R. Rogatto, I. Maesta, Placenta 2017, 50, 8-15.

[18] G. Li, C. Warden, Z. Zou, J. Neman, J. S. Krueger, A. Jain R. Jandial, M. Chen, PLoS One 2013, 8, e80970.

[19] N. Milosevich, M. C. Gignac, J. McFarlane, C. Simhadri, S. Horvath, K. D. Daze, C. S. Croft, A. Dheri, T. T. H. Quon, S. F. Douglas, J. E. Wulff, I. Paci, F. Hof, ACS Medicinal Chemistry Letters 2016, 7, 139-144.

[20] a) K. E. Denton, S. Wang, M. C. Gignac, N. Milosevich, F. Hof, E. C. Dykhuizen, C. J. Krusemark, SLAS DISCOVERY: Advancing Life Sciences R\&D 2018, 23, 417-428; b) S. Wang, K. E. Denton, K. F. Hobbs, T. Weaver, J. M. B. McFarlane, K. E. Connelly, M. C. Gignac, N. Milosevich, F. Hof, I. Paci, C. A. Musselman, E. C. Dykhuizen, C. J. Krusemark, ACS Chemical Biology 2019. [21] J. L. Suh, D. Bsteh, Y. Si, B. Hart, T. M. Weaver, C. Pribitzer, R. Lau, S. Soni, H. Ogana, J. M. Rectenwald, J. L. Norris, S. H. Cholensky, C. Sagum, J. D. Umana, D. Li, B. Hardy, M. T. Bedford, S. M. Mumenthaler, H.-J. Lenz, Y.m. Kim, G. G. Wang, K. H. Pearce, L. I. James, D. B. Kireev, 
C. A. Musselman, S. V. Frye, O. Bell, bioRxiv 2021, 2021.2002.2023.432388.

[22] N. Milosevich, J. McFarlane, M. C. Gignac, J. Li, T. M. Brown, C. R. Wilson, L. Devorkin, C. S. Croft, R. Hof, I. Paci, J. J. Lum, F. Hof, Bioorg Med Chem 2020, 28, 115176.

[23] X. Huang, J Biomol Screen 2003, 8, 34-38.

[24] J. I. Geller, J. J. Roth, J. A. Biegel, Crit Rev Oncog 2015, 20, 199-216.

[25] a) K. D. Barnash, K. N. Lamb, J. I. Stuckey, J. L. Norris, S. H. Cholensky, D. B. Kireev, S. V. Frye, L. I. James, ACS Chemical Biology 2016, 11, 2475-2483; b) L. Yang, Y. Liu, M. Fan, G. Zhu, H. Jin, J. Liang, Z. Liu, Z. Huang, L. Zhang, Eur J Med Chem 2019, 182, 111656. 


\section{Entry for the Table of Contents}

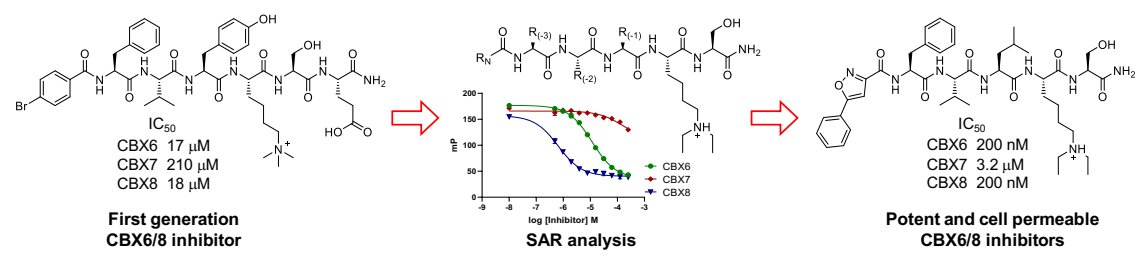

Dual active CBX inhibitor: A library of peptidomimetic based inhibitors for chromobox homolog 6 and 8 (CBX6/8) was developed. Through SAR key residues important for selectivity and potency were identified. We arrived at a dual active CBX6/8 inhibitor with sub micromolar affinity and 18-fold selectivity over CBX7. Preliminary cell studies show our compounds are cell permeable and have an effect on cell proliferation.

Institute and/or researcher Twitter usernames: @Hof_group_news 
Supplementary information for:

\section{Polycomb paralog chromodomain inhibitors active against both CBX6 and CBX8}

Natalia Milosevich, ${ }^{[a],[+]}$ Chelsea R. Wilson, ${ }^{[a],[+]}$ Tyler M. Brown, ${ }^{[a]}$ Aktan Alpsoy, ${ }^{[b]}$ Sijie Wang, ${ }^{[b]}$

Katelyn E. Connelly, ${ }^{[b]}$ Kirsten A. D. Sinclair, ${ }^{[a]}$ Felino R. Ponio, ${ }^{[a]}$ Rebecca Hof ${ }^{[a]}$ Emily C.

Dykhuizen, ${ }^{[\mathrm{b}]}$ and Fraser Hof ${ }^{*[\mathrm{a}]}$

\section{Table of Contents}

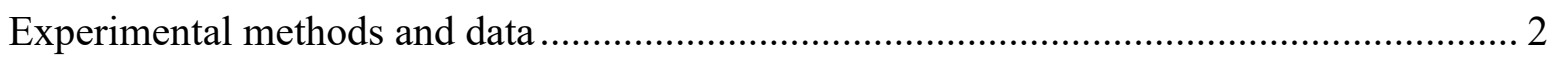

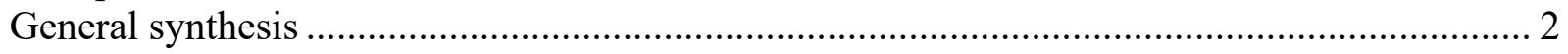

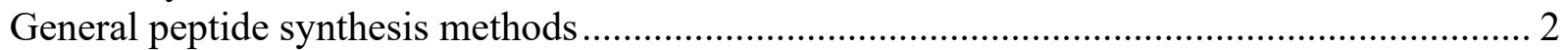

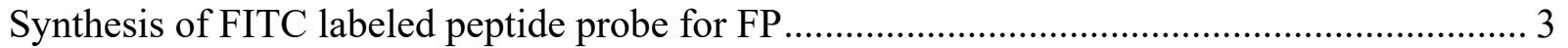

Synthesis of diethyl-lysine containing peptides......................................................... 4

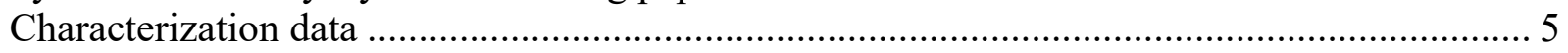

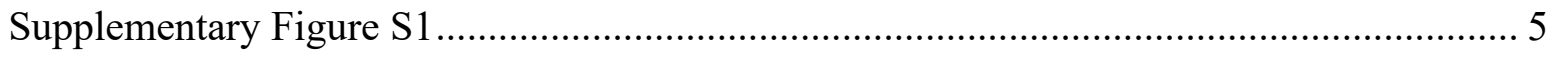

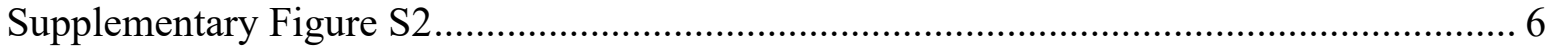

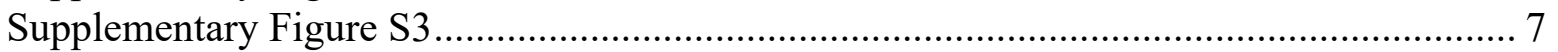

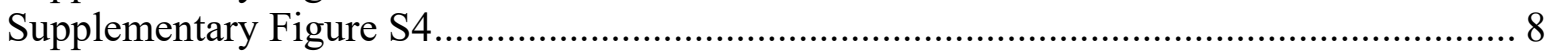

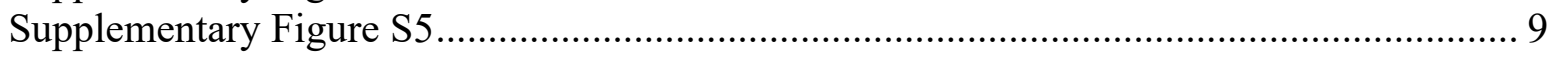

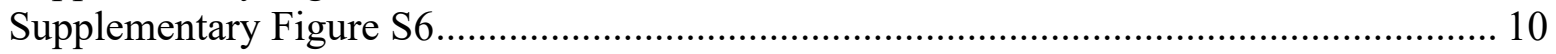

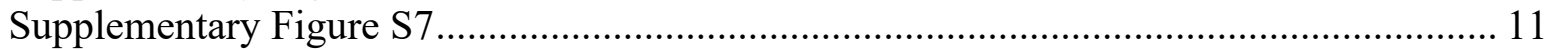

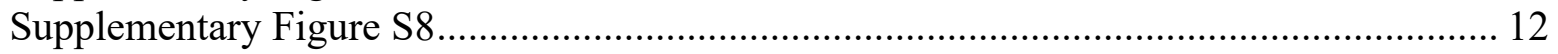

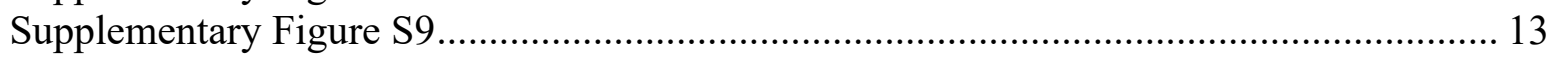

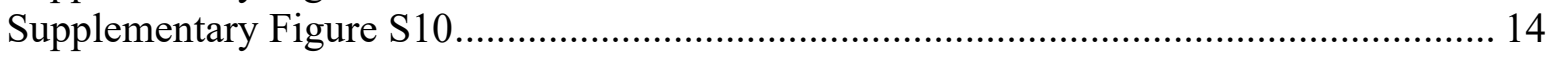

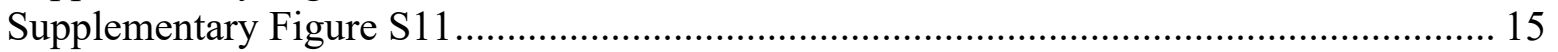

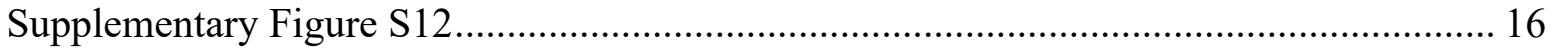

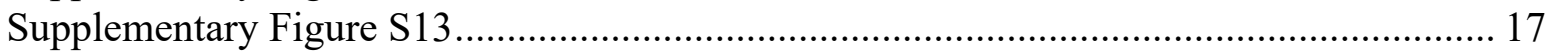

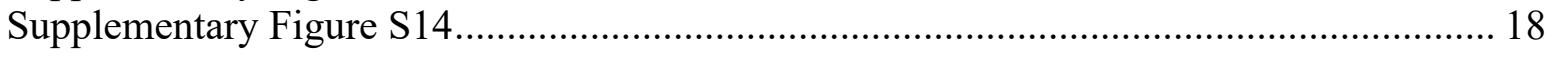

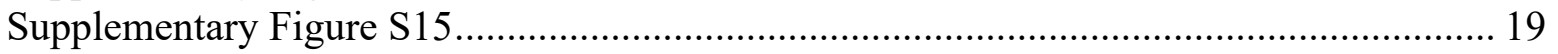

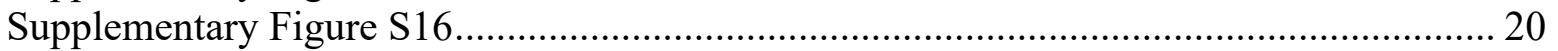

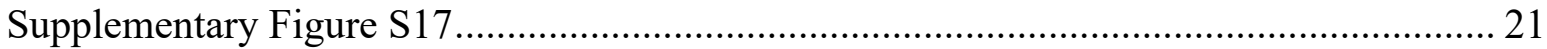

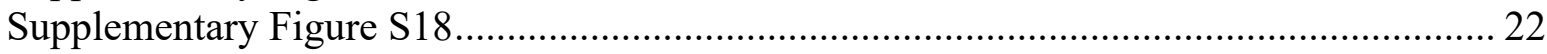

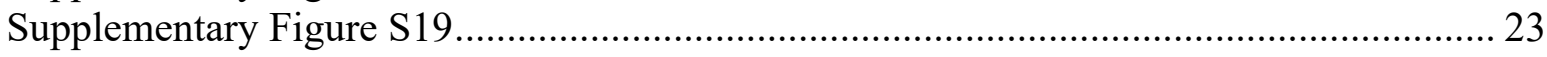

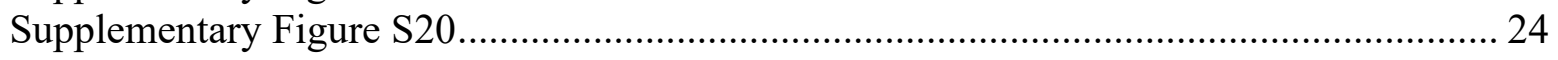

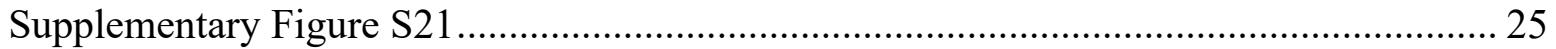

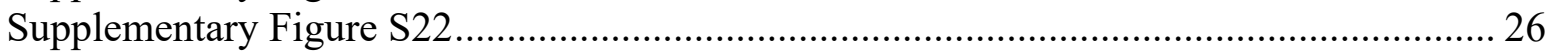

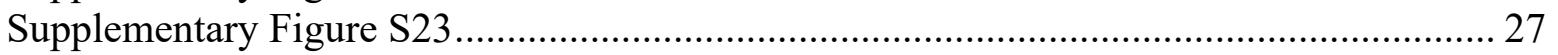

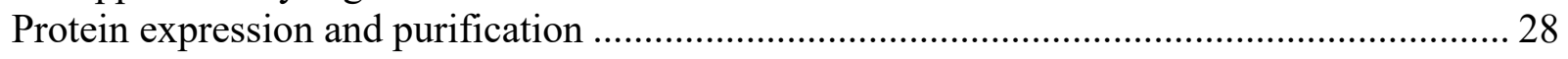

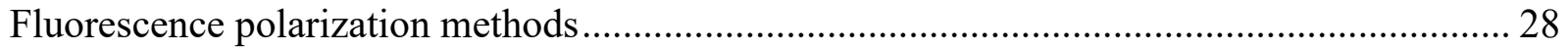

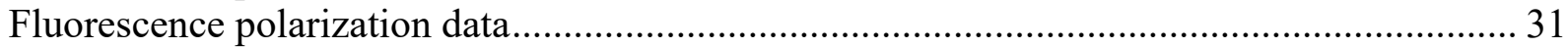

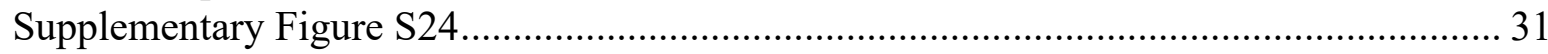

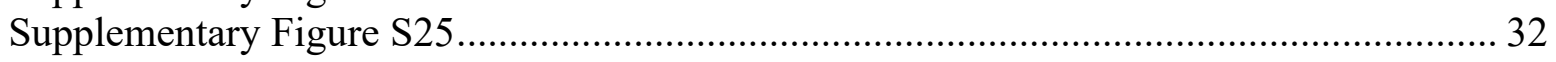




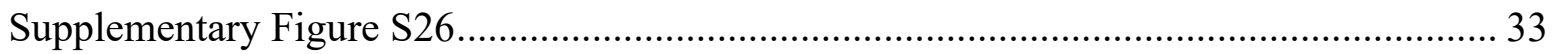

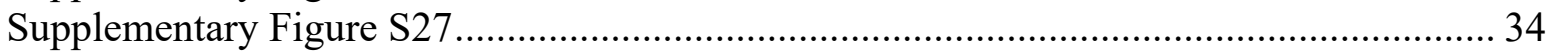

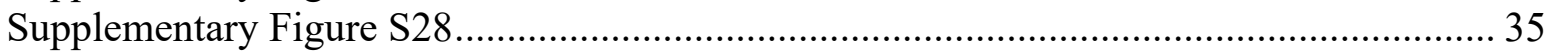

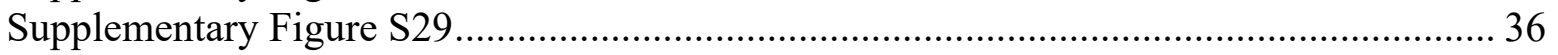

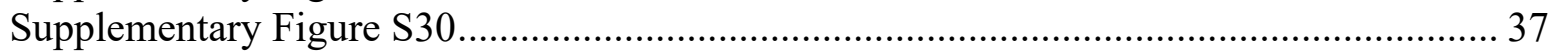

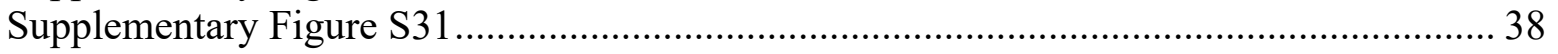

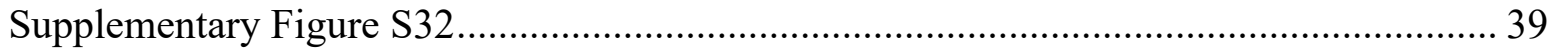

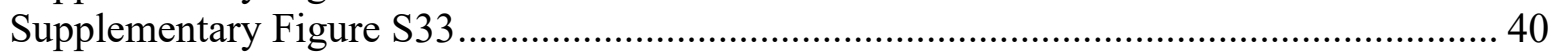

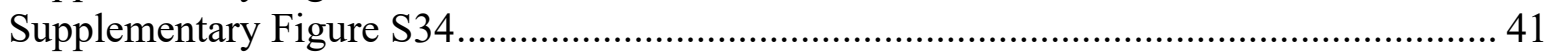

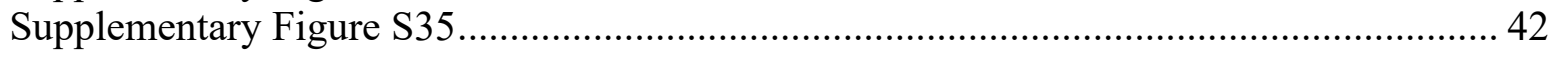

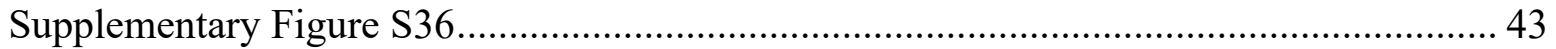

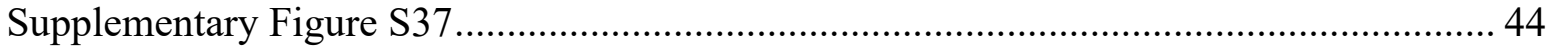

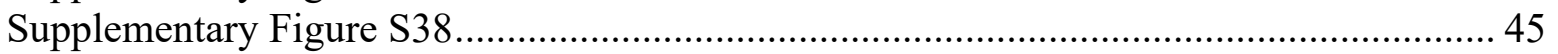

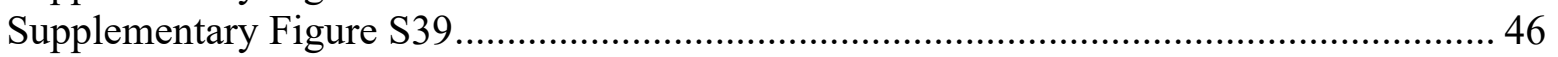

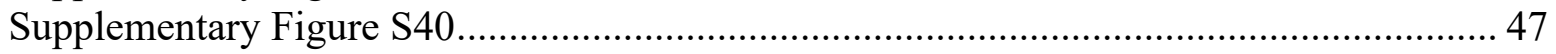

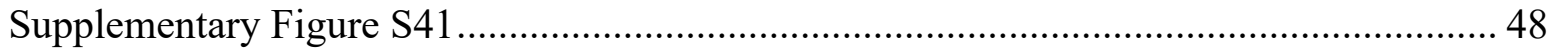

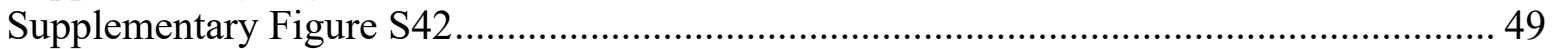

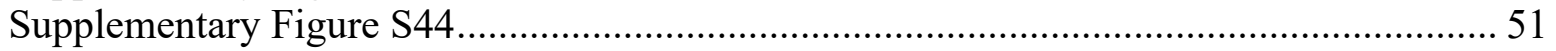

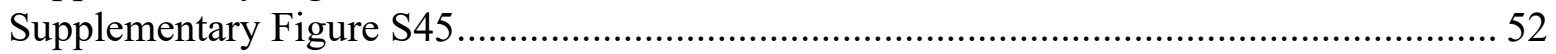

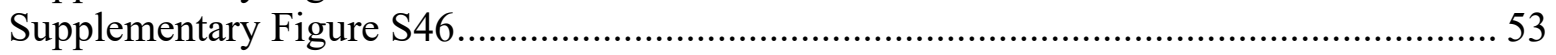

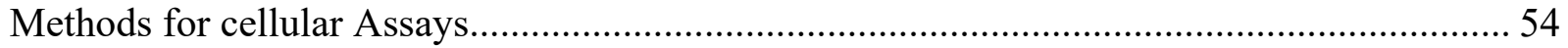

CellTiter-Glo luminescent cell viability assay ...................................................................... 54

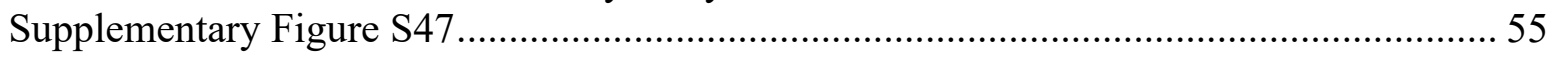

\section{Experimental methods and data}

\section{General synthesis}

All natural and unnatural amino acids and coupling agents were purchased from ChemImpex, Enamine and Sigma Aldrich. Acetylaldehyde and sodium cyanoborohydride were purchased from Sigma Aldrich and carboxylic acids used for $\mathrm{N}$-terminal modifications were purchased from Enamine, ChemImpex and Sigma Aldrich. Fmoc-L-Lys(Me) ${ }_{3}-\mathrm{OH}$ was purchased from GL Biochem (Shanghai).

\section{General peptide synthesis methods}

Peptides were either synthesized using an automated peptide synthesizer, or by manual peptide synthesis using Fmoc solid phase peptide synthesis protocols. Automated synthesis was achieved on Rink amide resin (ChemImpex International) using CEM Liberty-1 microwave synthesizer with standard Fmoc protocols using HBTU as the coupling agent. For manual peptide synthesis, Fmoc-protected Rink amide resin (ChemImpex International) was swelled in $\mathrm{CH}_{2} \mathrm{Cl}_{2}$ for 20 minutes. Deprotection of Fmoc was done using 20\% piperidine in DMF $(10 \mathrm{~mL}, 5$ minutes, 3 
times) and washed with DMF (10 mL, 3 times following each incubation). Fmoc- and side chain protected amino acids as well as N-capping carboxylic acids (5 eq.) were dissolved in DMF (10 mL) with HBTU (5 eq.), and DIPEA (10 eq.), added to the Fmoc deprotected resin and/or Fmoc deprotected peptide on resin, bubbled with $\mathrm{N}_{2}$ for one hour at room temperature. The resin was washed with DMF (10 mL, 3 times), and washed with $\mathrm{CH}_{2} \mathrm{Cl}_{2}(10 \mathrm{~mL}, 3$ times $)$ after the final coupling.

Peptide products were cleaved from resin with $10 \mathrm{~mL}$ of 95:2.5:2.5 TFA/ $\mathrm{H}_{2} \mathrm{O} /$ triisopropylsilane for $2.5 \mathrm{~h}$. The solution was then concentrated in vacuo and added to cold diethyl ether to yield a crude yellow precipitate that was collected by centrifugation. Purifications by preparative HPLC were carried out using a Phenomenex Luna, $5 \mu \mathrm{m}, \mathrm{C}-18$ column, 250 x $21.20 \mathrm{~mm}$ column. Peptides were characterized using UPLC, HPLC, and ESI-MS and purity was determined to be $>95 \%$ by analytical HPLC or analytical UPLC (exceptions are noted in the characterization data). Retention times reported arise from analytical traces done on either a Thermo Scientific C18 column (4.6 mm x 250 mm, $5 \mu \mathrm{m})$, Waters UPLC-MS Acquity UPLC BEH C18 column (2.1 x $50 \mathrm{~mm}, 1.7 \mu \mathrm{M})$, or Shimadzu HPLC C-18 column (4.6 mm x $250 \mathrm{~mm}, 5 \mu \mathrm{m})$. Traces done on the Thermo or Shimadzu HPLC were at a flow rate of $1.5 \mathrm{~mL} / \mathrm{min}$, gradient running from 90:10 water $(0.1 \%$ TFA $)$ and $\operatorname{MeCN}(0.1 \%$ TFA $)$ to $10: 90$ water $(0.1 \%$ TFA $)$ and $\mathrm{MeCN}(0.1 \%$ TFA) over 30-38 min. Traces done on the Waters UPLC-MS were at a flow rate of $0.5 \mathrm{~mL} / \mathrm{min}$ with a gradient running from 90:10 water $(0.1 \%$ TFA) and $\operatorname{MeCN}(0.1 \%$ TFA) to 10:90 water $(0.1 \%$ TFA $)$ and $\mathrm{MeCN}(0.1 \%$ TFA $)$ over five minutes.

\section{Synthesis of FITC labeled peptide probe for FP}

For side-chain functionalization with fluorescein isothiocyanate (FITC), a side-chain (Mtt)protected lysine was used. For functionalization of side-chain Mtt-protected lysine, the Mtt protecting group was cleaved after complete synthesis on resin. Selective cleavage was achieved with treatment of 2:2:96 TFA/triisopropylsilane $/ \mathrm{CH}_{2} \mathrm{Cl}_{2}$ for 20 minutes $(\times 4)$. The resin was filtered and washed with $\mathrm{CH}_{2} \mathrm{Cl}_{2}(3 \times 5 \mathrm{~mL})$ and DMF $(3 \times 5 \mathrm{~mL})$. FITC ( 5 equiv) was added to a $10 \mathrm{~mL}$ solution of 1:1 DMF and pyridine. This solution was added to the resin and bubbled with $\mathrm{N}_{2}$ at ambient temperature overnight. Following the functionalization with FITC, all 
peptide/resin solutions were filtered and the resin washed with DMF $(3 \times 5 \mathrm{~mL})$ and $\mathrm{CH}_{2} \mathrm{Cl}_{2}(3 \times$ $5 \mathrm{~mL}$ ) and air-dried.

\section{Synthesis of diethyl-lysine containing peptides}

Side-chain (Mtt)-protected lysine was used in the synthesis protocols, and cleaved as described previously in Chapter 2, section 2.8.2 (treatment with 2:2:96 TFA/triisopropylsilane $/ \mathrm{CH}_{2} \mathrm{Cl}_{2}$ for 20 minutes, 4 times). The resin was filtered and washed with $\mathrm{CH}_{2} \mathrm{Cl}_{2}(3 \times 5 \mathrm{~mL})$ and DMF (3 x 5 $\mathrm{mL}$ ). A solution of acetylaldehyde (50-100 eq.), sodium cyanoborohydride (10 eq.), and 1 drop of glacial acetic acid in DMF $(10 \mathrm{~mL})$ was added to the peptide on resin. The solution was filtered and the resin washed with DMF $(3 \times 5 \mathrm{~mL})$ and $\mathrm{CH}_{2} \mathrm{Cl}_{2}(3 \times 5 \mathrm{~mL})$ and air-dried. Crude peptides were dissolved in a mixture of $\mathrm{H}_{2} \mathrm{O}$ and $\mathrm{MeCN}$ or DMSO, depending on solubility. Peptide cleavage from resin and purification are the same as reported above in section 4.7.3. Diethyl peptides with poor solubility were purified from DMSO stocks with a gradient running from 60:40 water (0.1 \% TFA) and $\operatorname{MeCN}(0.1 \%$ TFA) to 10:90 water (0.1\% TFA) and $\mathrm{MeCN}$ (0.1\% TFA) over 30-38 min. 


\section{Characterization data}

A)

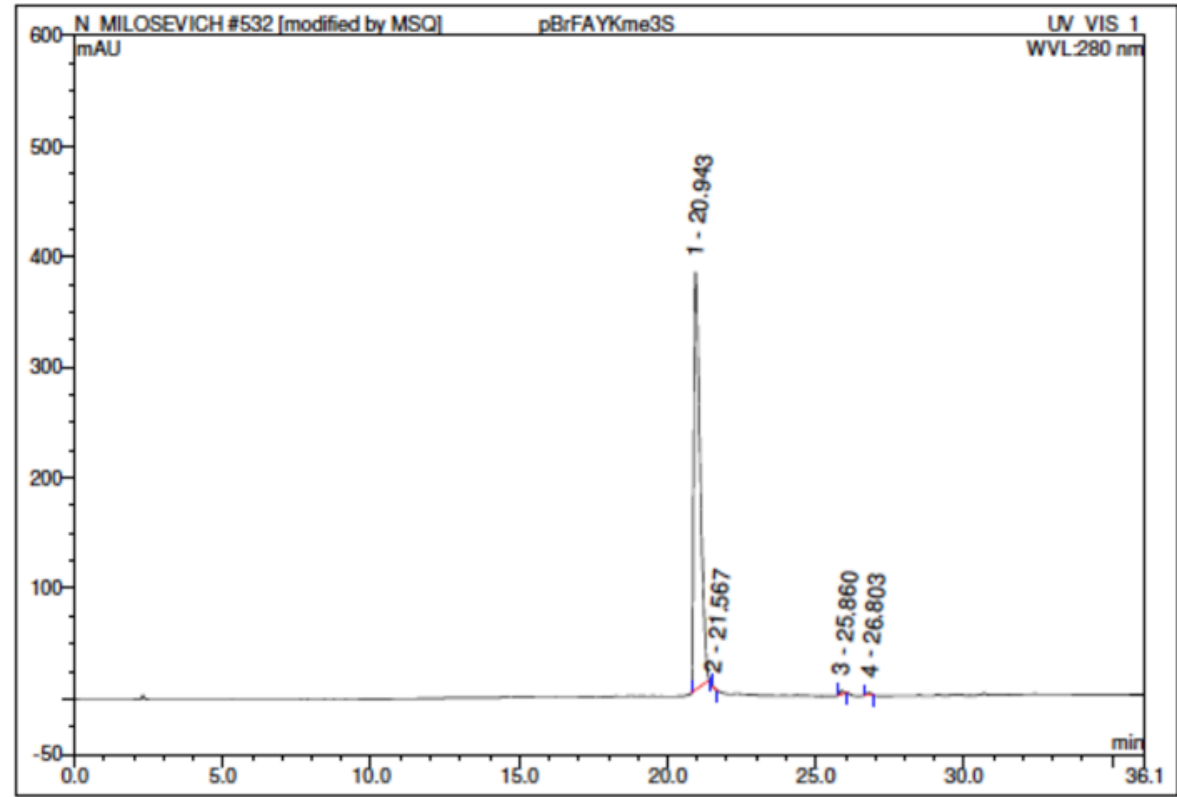

\begin{tabular}{|r|ccrrrrr|}
\hline No. & $\begin{array}{c}\text { Ret.Time } \\
\text { min }\end{array}$ & Peak Name & $\begin{array}{c}\text { Height } \\
\text { mAU }\end{array}$ & $\begin{array}{c}\text { Area } \\
\text { mAU*min }\end{array}$ & $\begin{array}{c}\text { Rel.Area } \\
\%\end{array}$ & $\begin{array}{c}\text { Amount } \\
\mu l\end{array}$ & Type \\
\hline 1 & 20.94 & n.a. & 377.219 & 94.395 & 99.05 & n.a. & BMB* $^{*}$ \\
2 & 21.57 & n.a. & 0.474 & 0.023 & 0.02 & n.a. & BMB* $^{*}$ \\
3 & 25.86 & n.a. & 3.386 & 0.466 & 0.49 & n.a. & BMB* $^{*}$ \\
4 & 26.80 & n.a. & 2.205 & 0.420 & 0.44 & n.a. & BMB* $^{*}$ \\
\hline Total: & & & 383.285 & 95.304 & 100.00 & 0.000 & \\
\hline
\end{tabular}

B)

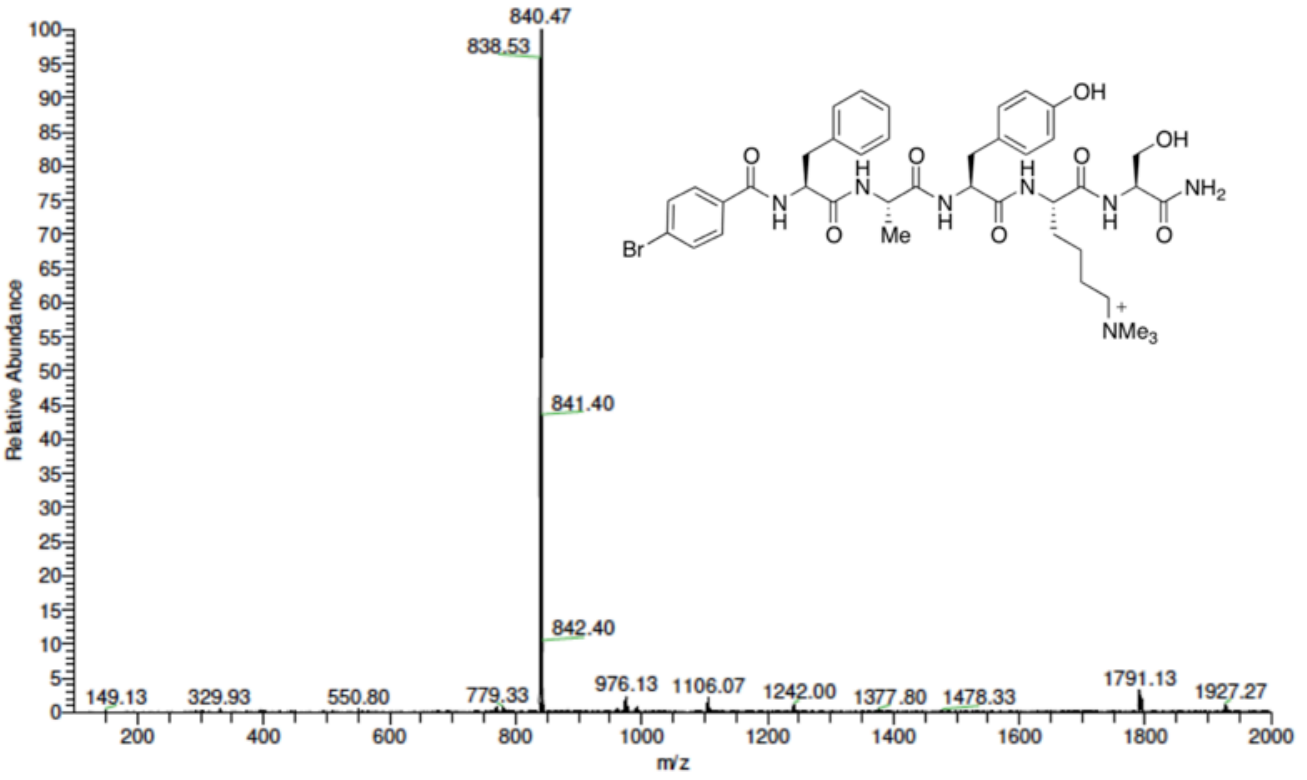

Supplementary Figure S1: Characterization data for compound 1. A) Analytical HPLC trace, B) Low resolution mass spectrum. LR-ESI-MS: $[m / z]$ calcd. for $\mathrm{C}_{40} \mathrm{H}_{53} \mathrm{BrN}_{7} \mathrm{O}^{+}$: 838.31; found: 838.53 . 
A)

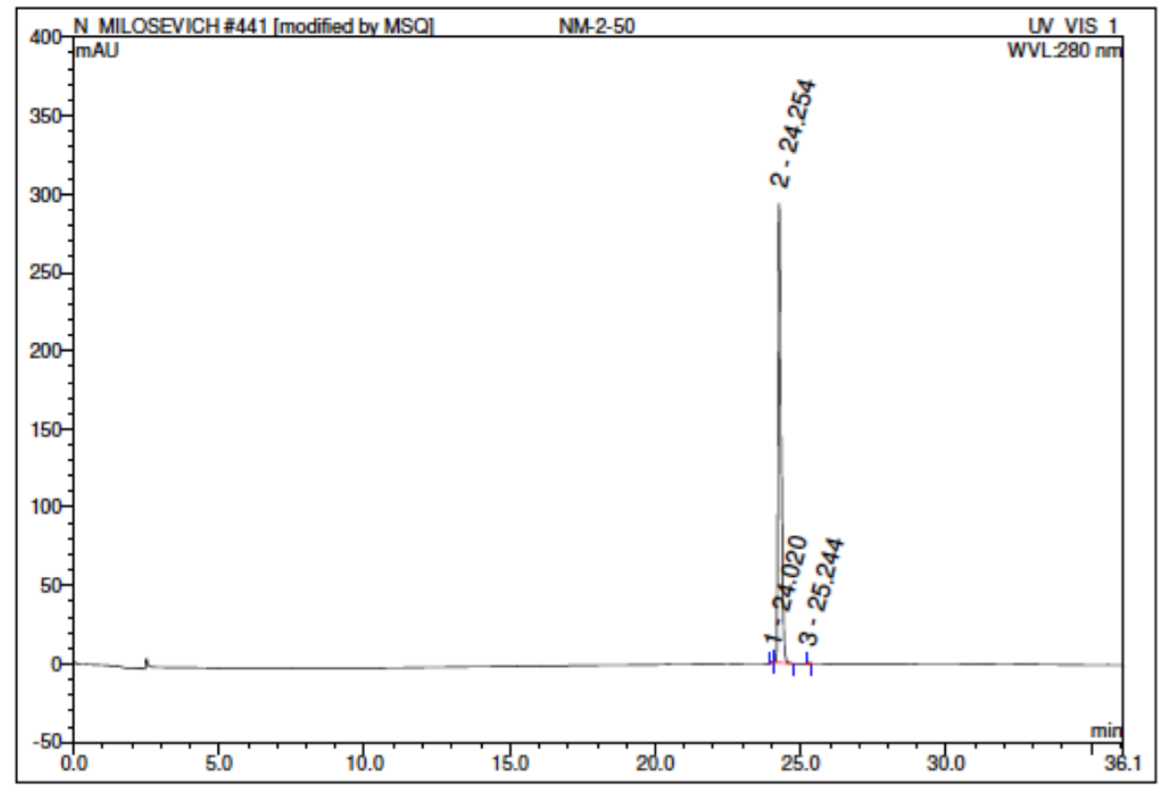

\begin{tabular}{|c|c|c|c|c|c|c|c|}
\hline No. & $\begin{array}{c}\text { Ret.Time } \\
\text { min }\end{array}$ & Peak Name & $\begin{array}{c}\text { Height } \\
\text { mAU }\end{array}$ & $\begin{array}{c}\text { Area } \\
\text { mAU*min }\end{array}$ & $\begin{array}{c}\text { Rel.Area } \\
\%\end{array}$ & $\begin{array}{c}\text { Amount } \\
\mu \mathrm{l}\end{array}$ & Type \\
\hline 1 & 24.02 & n.a. & 0.664 & 0.058 & 0.16 & n.a. & $\mathrm{BMb}^{*}$ \\
\hline 2 & 24.25 & n.a. & 292.917 & 37.070 & 99.78 & n.a. & $\mathrm{bMB}^{*}$ \\
\hline 3 & 25.24 & n.a. & 0.384 & 0.024 & 0.06 & n.a. & BMB* $^{*}$ \\
\hline Total: & & & 293.965 & 37.152 & 100.00 & 0.000 & \\
\hline
\end{tabular}

B)

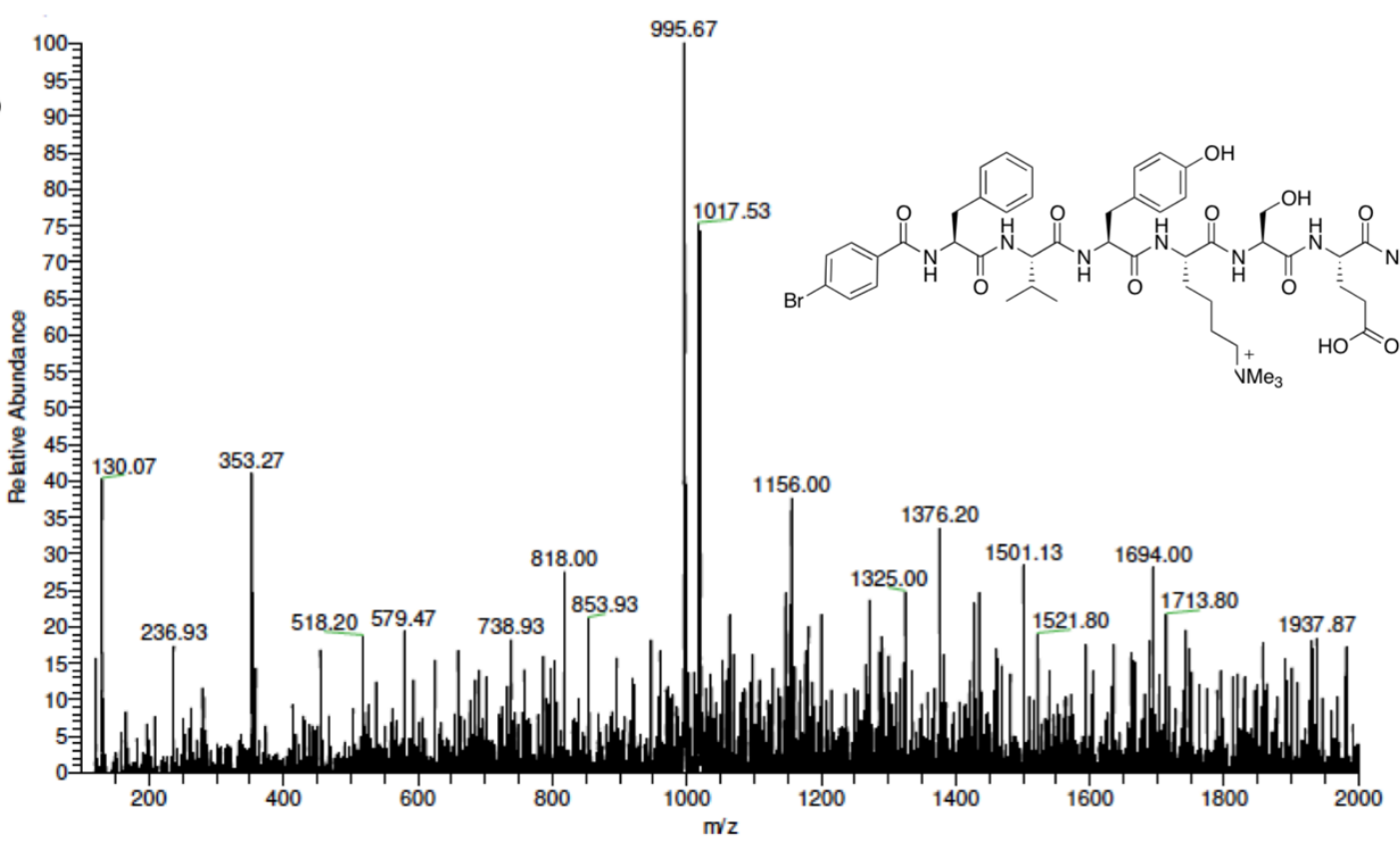

Supplementary Figure S2: Characterization data for compound 5. A) Analytical HPLC trace, B) Low resolution mass spectrum. LR-ESI-MS: [m/z] calcd. for $\mathrm{C}_{47} \mathrm{H}_{64} \mathrm{BrN}_{8} \mathrm{O}_{11}{ }^{+}$: 995.39; found: 995.67. 
A) Chromatogram

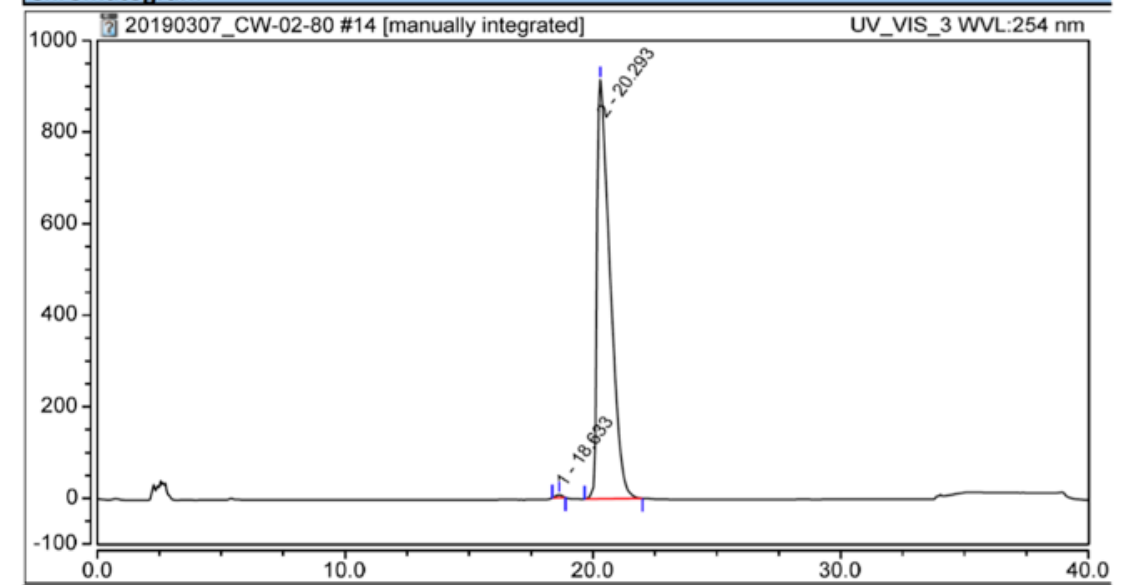

\begin{tabular}{|l|c|c|c|c|c|}
\hline \multicolumn{2}{|l|}{ Integration Results } \\
\hline No. & $\begin{array}{c}\text { Retention Time } \\
\min \end{array}$ & $\begin{array}{c}\text { Area } \\
\text { mAU*min }\end{array}$ & $\begin{array}{c}\text { Height } \\
\text { mAU }\end{array}$ & $\begin{array}{c}\text { Relative Area } \\
\%\end{array}$ & $\begin{array}{c}\text { Relative Height } \\
\%\end{array}$ \\
\hline 1 & 18.633 & 2.073 & 6.728 & 0.37 & 0.73 \\
2 & 20.293 & 557.845 & 916.418 & 99.63 & 99.27 \\
\hline Total: & 559.919 & 923.146 & 100.00 & 100.00 \\
\hline
\end{tabular}

B)

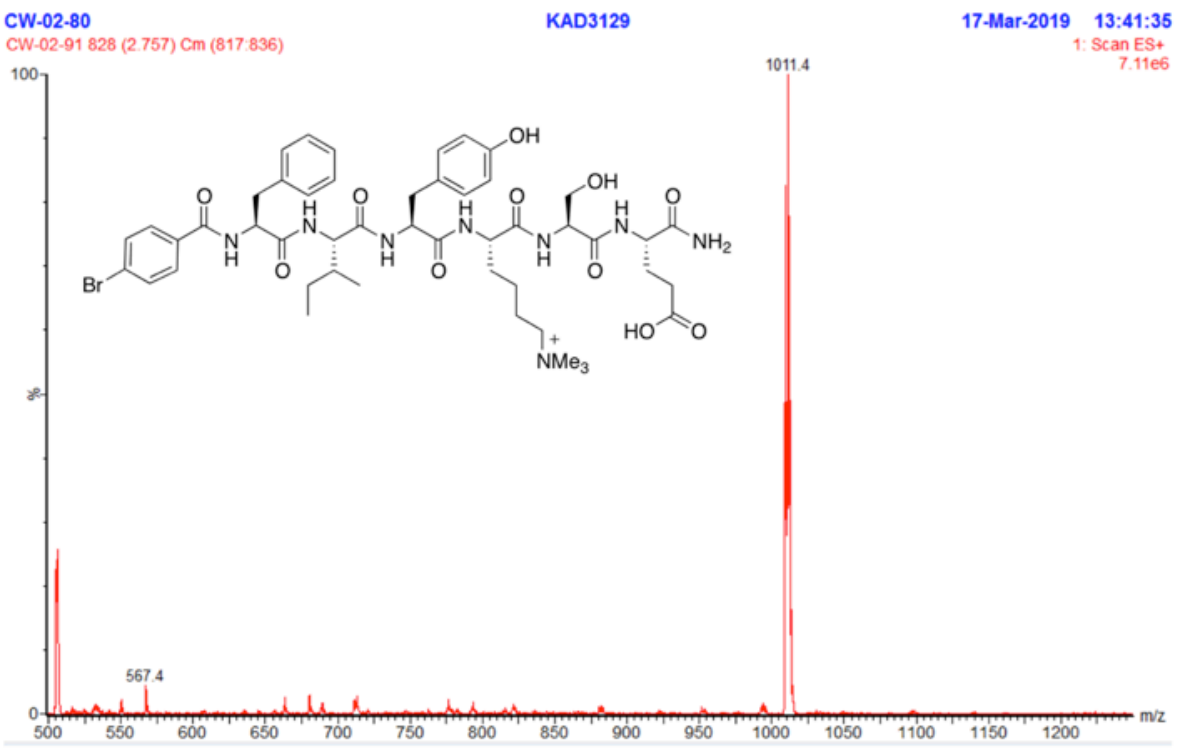

Supplementary Figure S3: Characterization data for compound 6. A) Analytical HPLC trace, B) Low resolution mass spectrum. LR-ESI-MS: $[\mathrm{m} / z]$ calcd. For $\mathrm{C}_{48} \mathrm{H}_{66} \mathrm{BrN}_{8} \mathrm{O}_{11}{ }^{+}: 1011.41$; found: 1011.4. 
A)

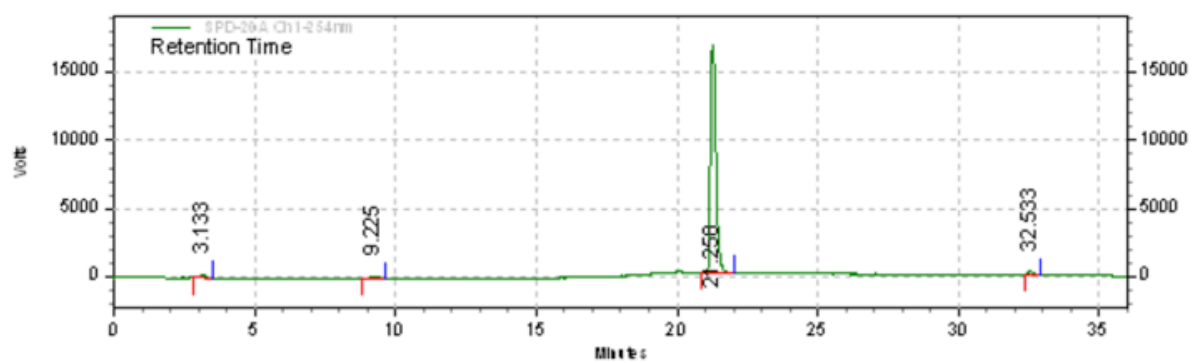

SPD-20A

Chl-254rum

Results

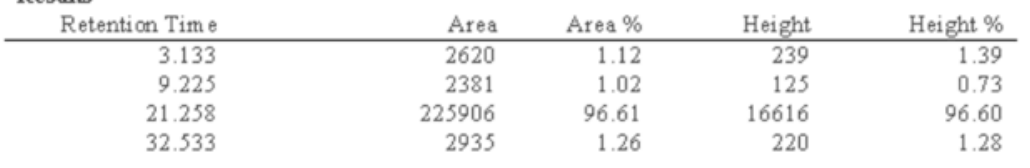

B)

\begin{tabular}{|r|r|r|r|r|}
\hline Totals & 233842 & 100.00 & 17200 & 100.00 \\
\hline
\end{tabular}

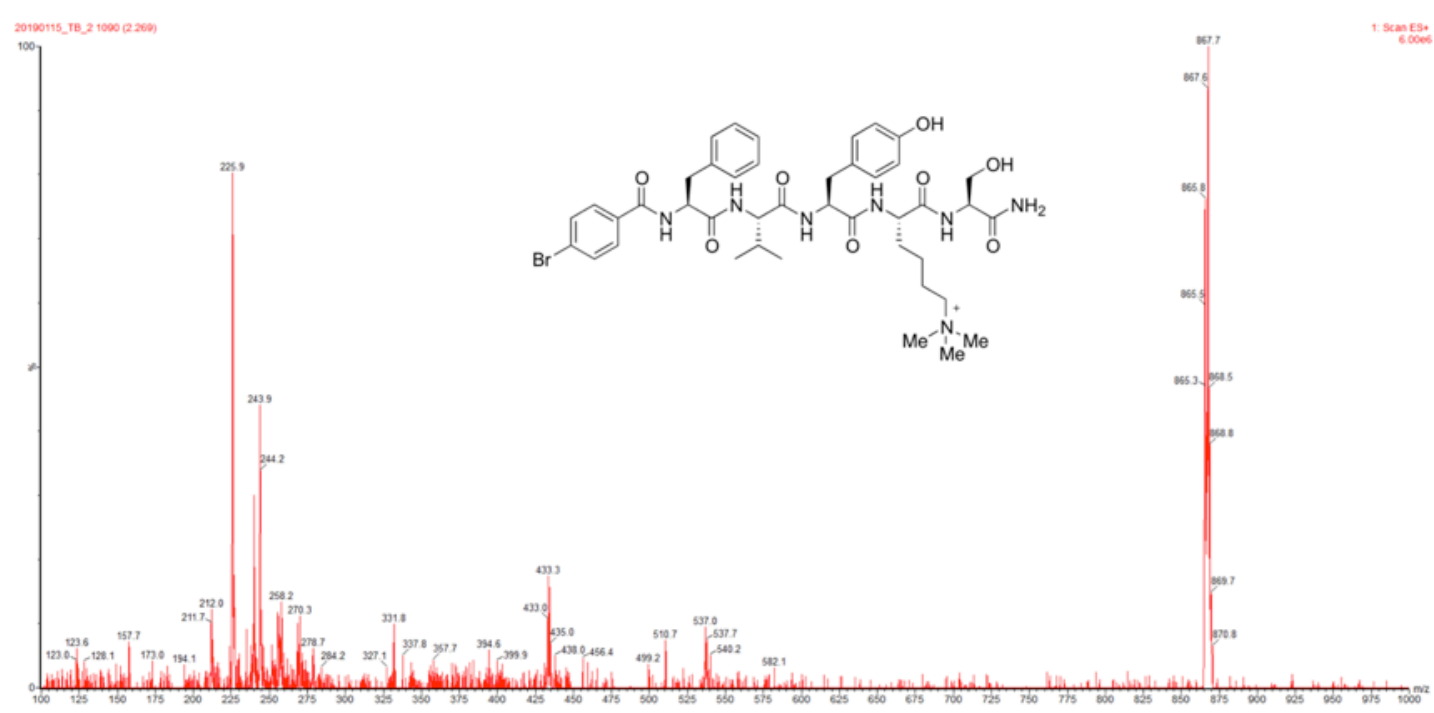

Supplementary Figure S4: Characterization data for compound 7. A) Analytical HPLC trace, B) Low resolution mass spectrum. LR-ESI-MS: $[\mathrm{m} / z]$ calcd. for $\mathrm{C}_{42} \mathrm{H}_{57} \mathrm{BrN}_{7} \mathrm{O}_{8}{ }^{+}: 867.85$; found: 867.7 . 
A)

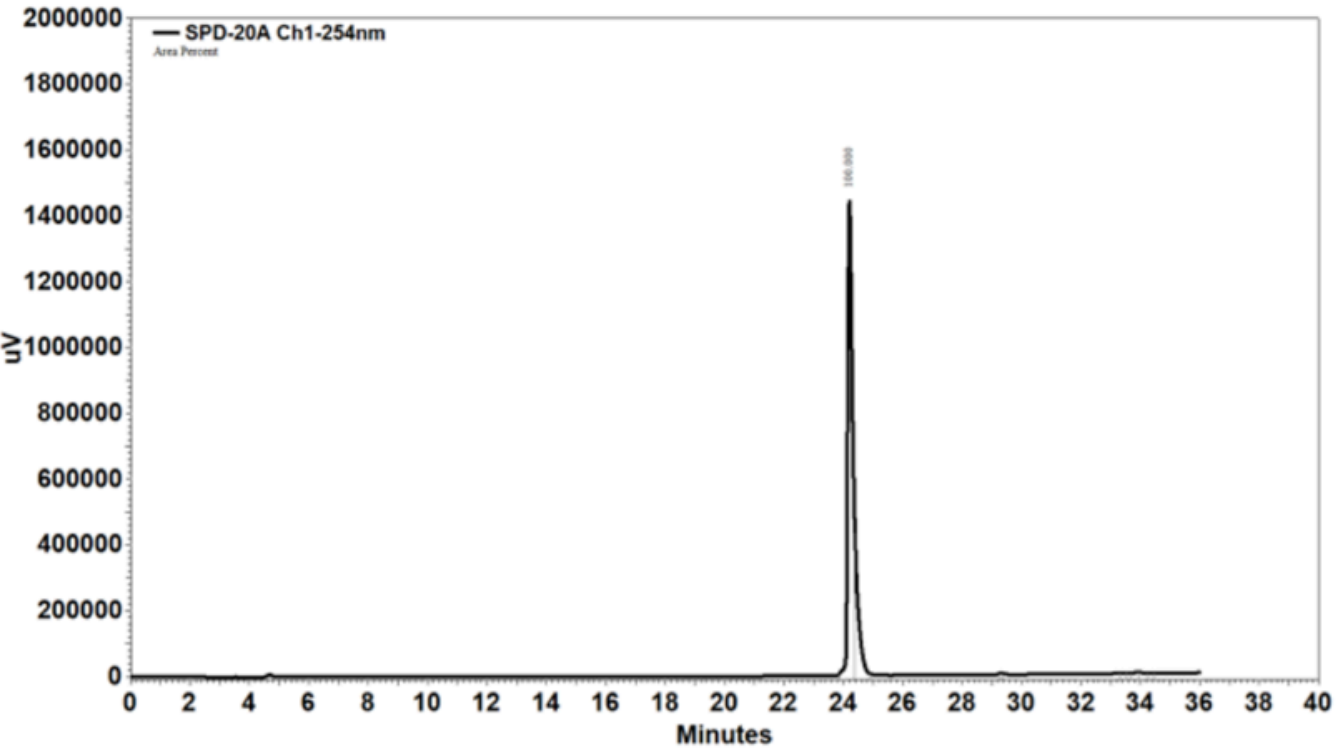

B)

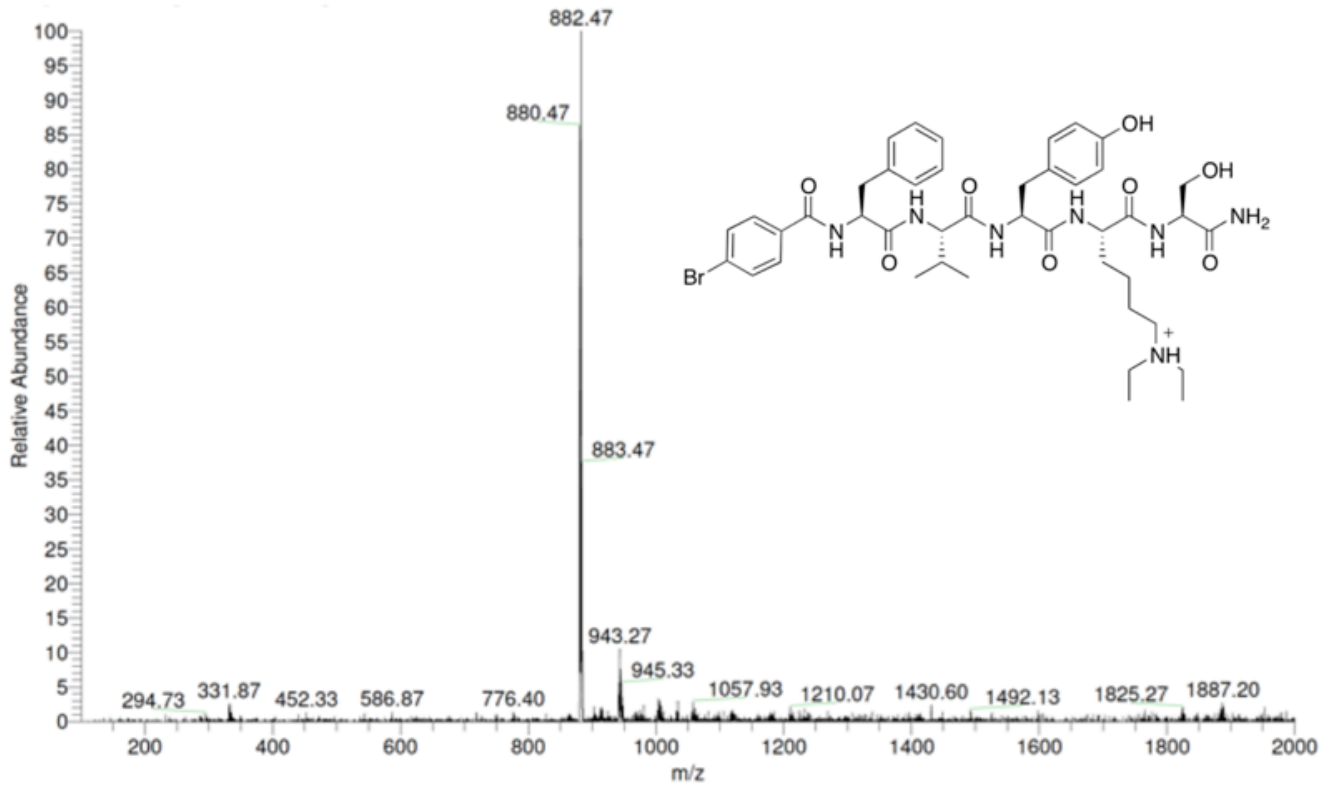

Supplementary Figure S5: Characterization data for compound 8. A) Analytical HPLC trace, B) Low resolution mass spectrum. LR-ESI-MS: $[\mathrm{m} / z]$ calcd. for: $\mathrm{C}_{43} \mathrm{H}_{58} \mathrm{BrN}_{7} \mathrm{O}_{8}{ }^{+}$: 880.36; found: 880.47 . 


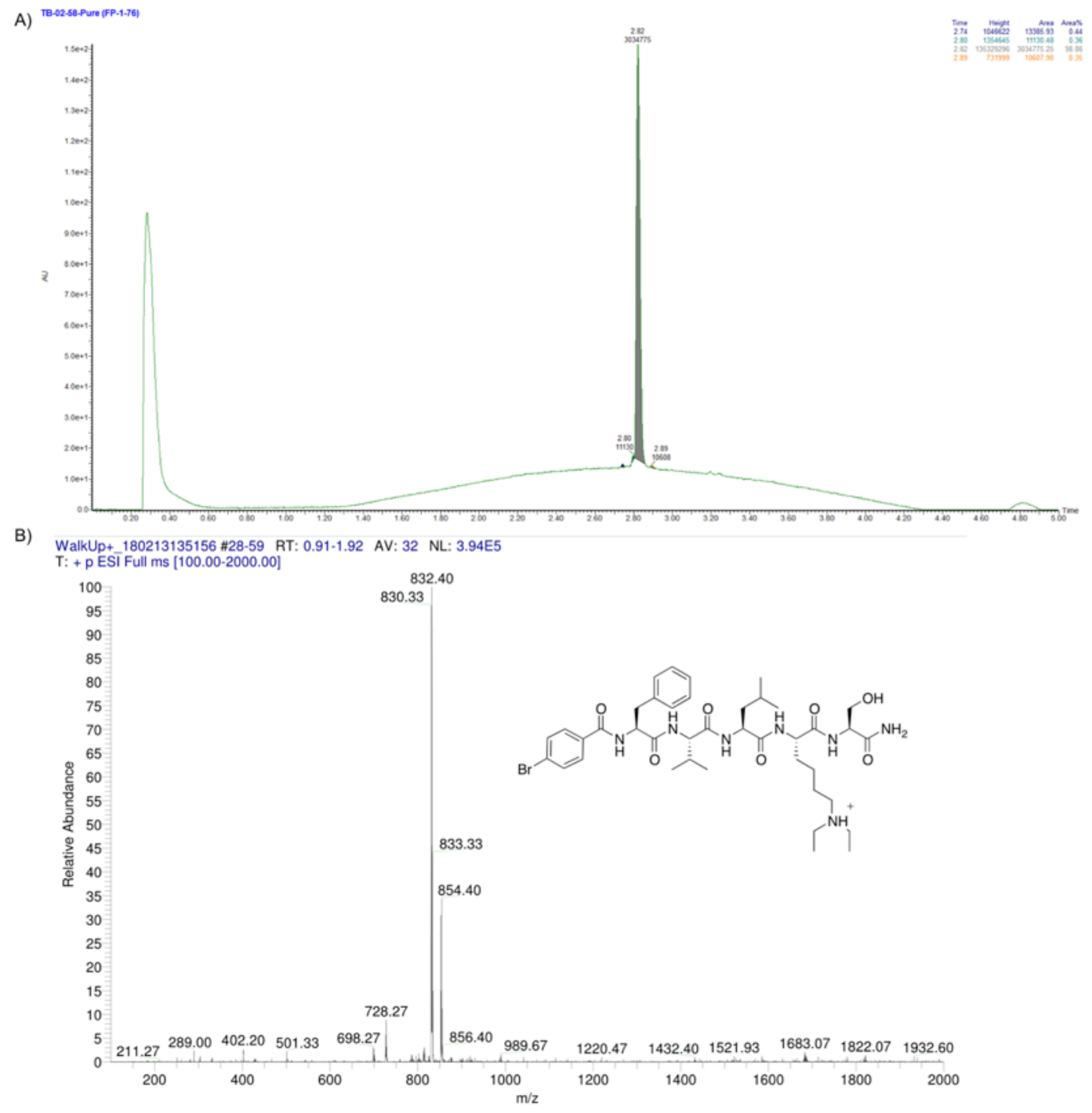

Supplementary Figure S6: Characterization data for compound 9. A) Analytical UPLC trace. Product peak is at 2.82 minutes with area of $98 \%$. B) Low resolution mass spectrum. LR-ESIMS: [m/z] calcd. for: $\mathrm{C}_{40} \mathrm{H}_{61} \mathrm{BrN}_{7} \mathrm{O}_{7}^{+}$: 830.38; found: 830.33 . 
A)

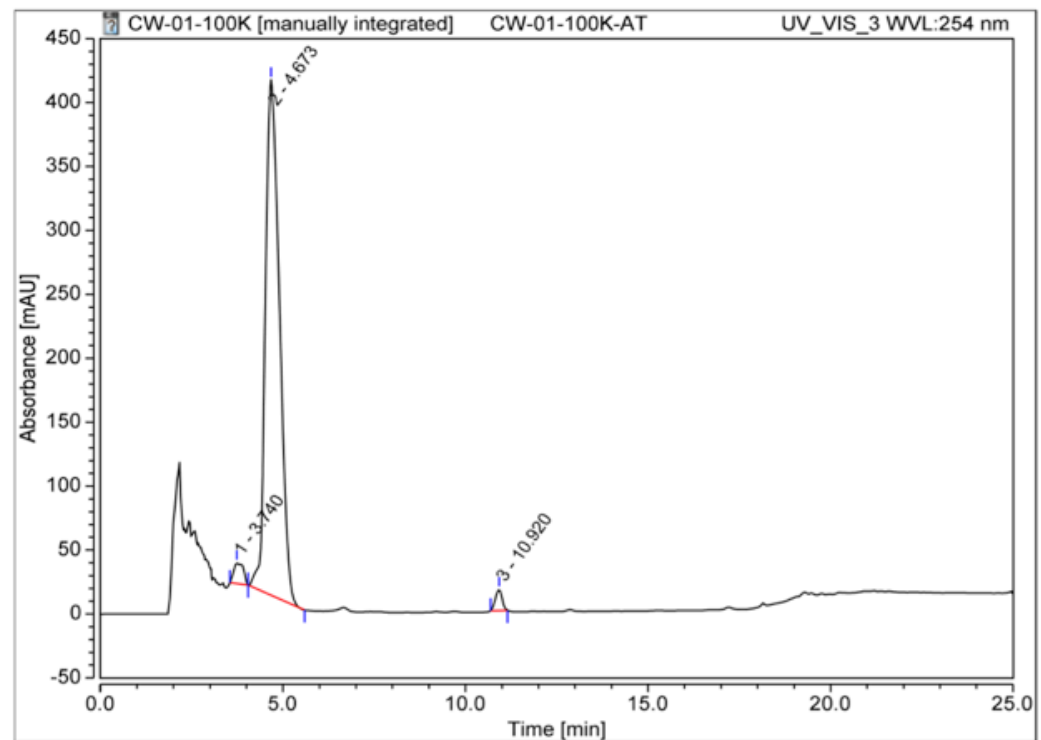

B)

\begin{tabular}{|l|c|c|c|c|c|}
\hline \hline No. & $\begin{array}{c}\text { Retention Time } \\
\min \end{array}$ & $\begin{array}{c}\text { Area } \\
\text { mAU*min }\end{array}$ & $\begin{array}{c}\text { Height } \\
\text { mAU }\end{array}$ & $\begin{array}{c}\text { Relative Area } \\
\%\end{array}$ & $\begin{array}{c}\text { Relative Height } \\
\%\end{array}$ \\
\hline 1 & 3.740 & 5.061 & 16.235 & 2.69 & 3.73 \\
2 & 4.673 & 179.676 & 403.079 & 95.48 & 92.51 \\
3 & 10.920 & 3.445 & 16.411 & 1.83 & 3.77 \\
\hline
\end{tabular}

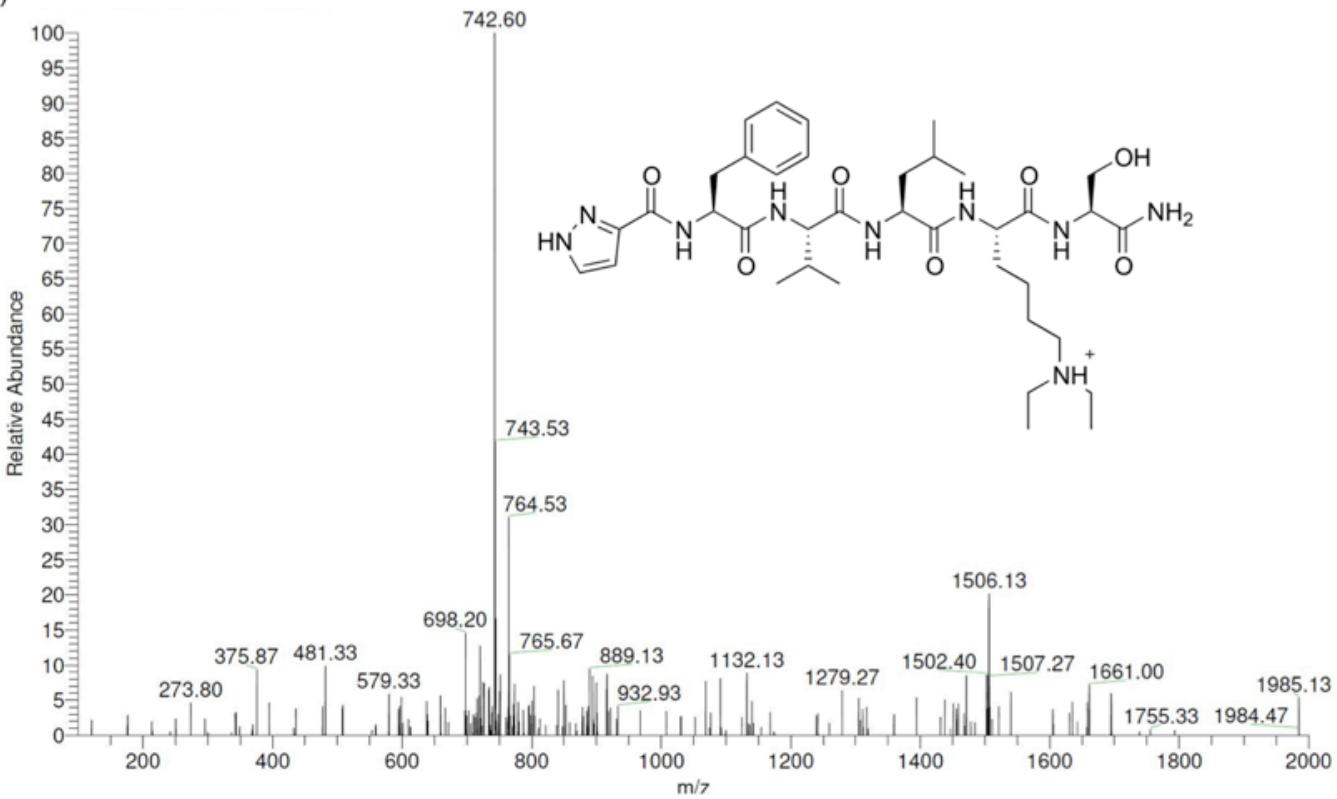

Supplementary Figure S7: Characterization data for compound 10. A) Analytical HPLC trace, B) Low resolution mass spectrum. LR-ESI-MS: $[\mathrm{m} / z]$ calcd. for: $\mathrm{C}_{37} \mathrm{H}_{60} \mathrm{~N}_{9} \mathrm{O}_{7}^{+}:$: 742.46; found: 742.60 . 
A) Chromatogram

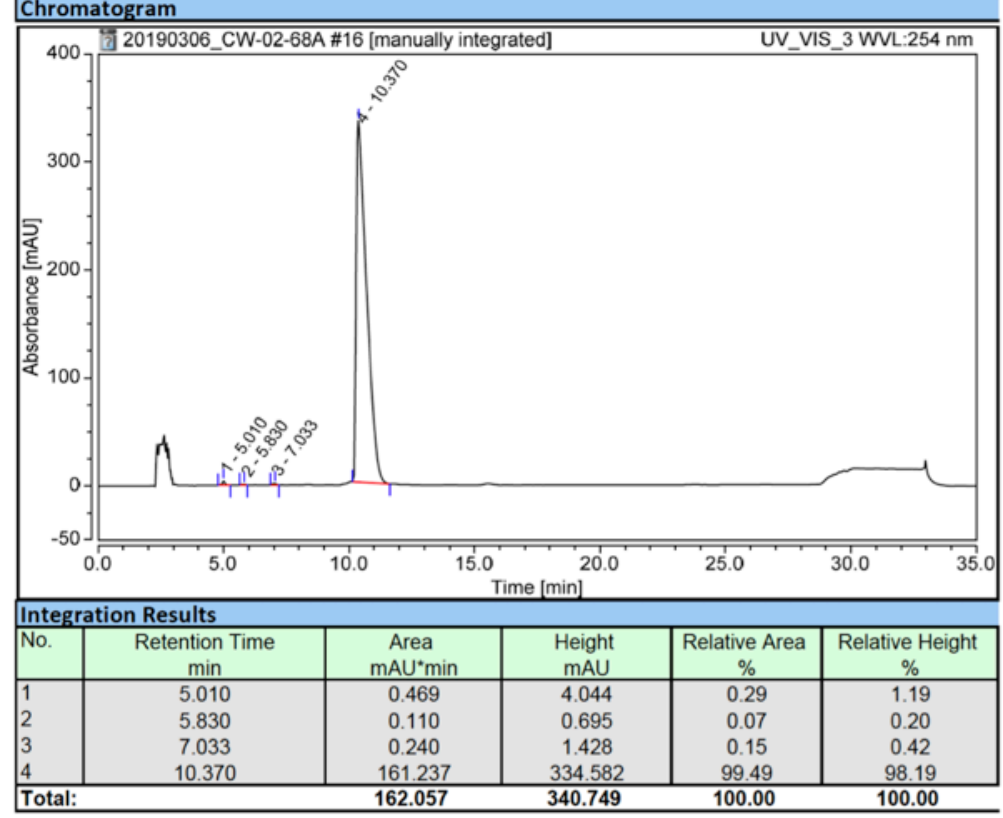

B) WalkUp+_180216105813 \#49-61 RT: 1.60-2.00 AV: 13 NL: $2.98 E 5$

T: + p ESİ Full ms [100.00-2000.00]

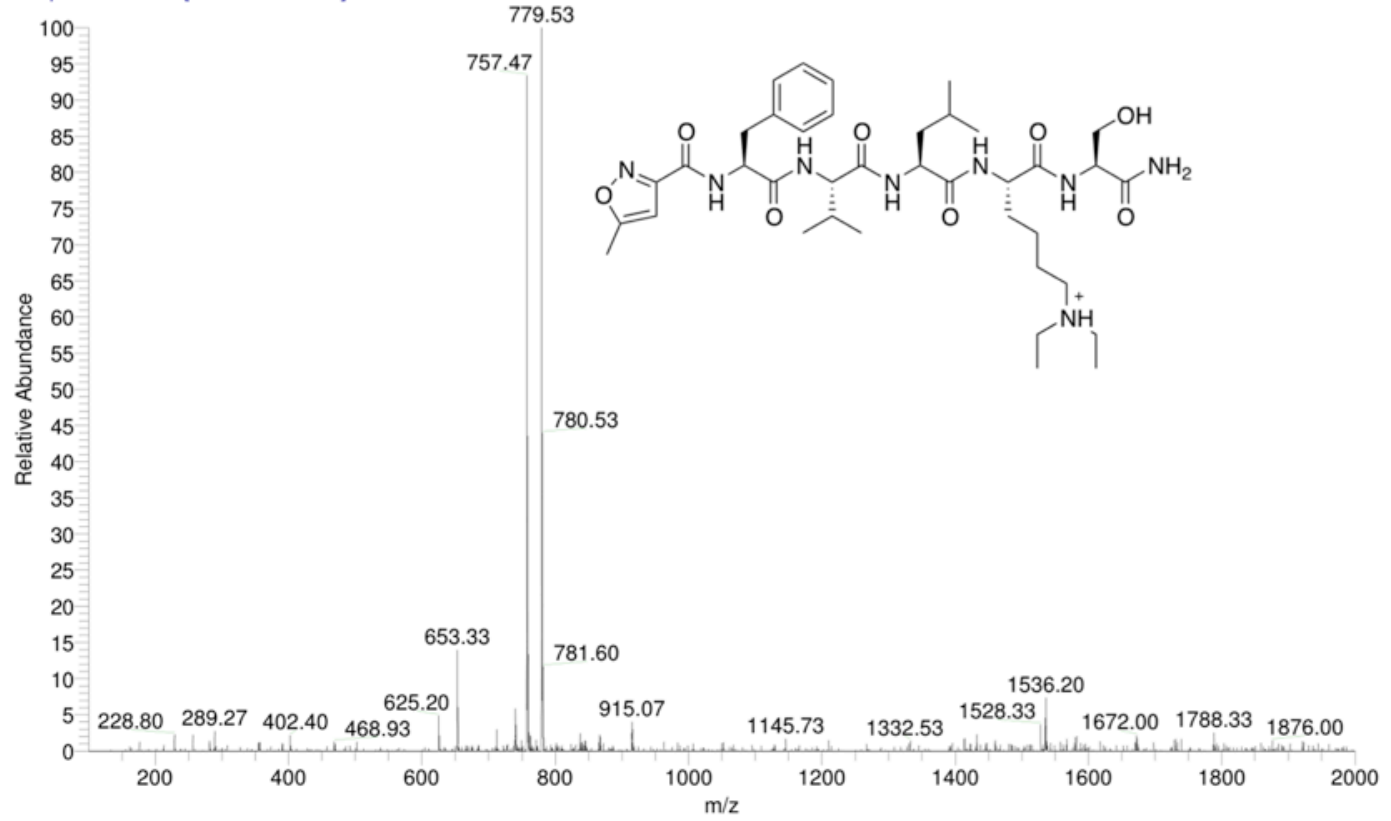

Supplementary Figure S8: Characterization data for compound 11. A) Analytical HPLC trace, B) Low resolution mass spectrum. LR-ESI-MS: $[\mathrm{m} / z]$ calcd. for: $\mathrm{C}_{38} \mathrm{H}_{61} \mathrm{~N}_{8} \mathrm{O}_{8}{ }^{+}: 757.46$; found: 757.47 
A) Chromatogram

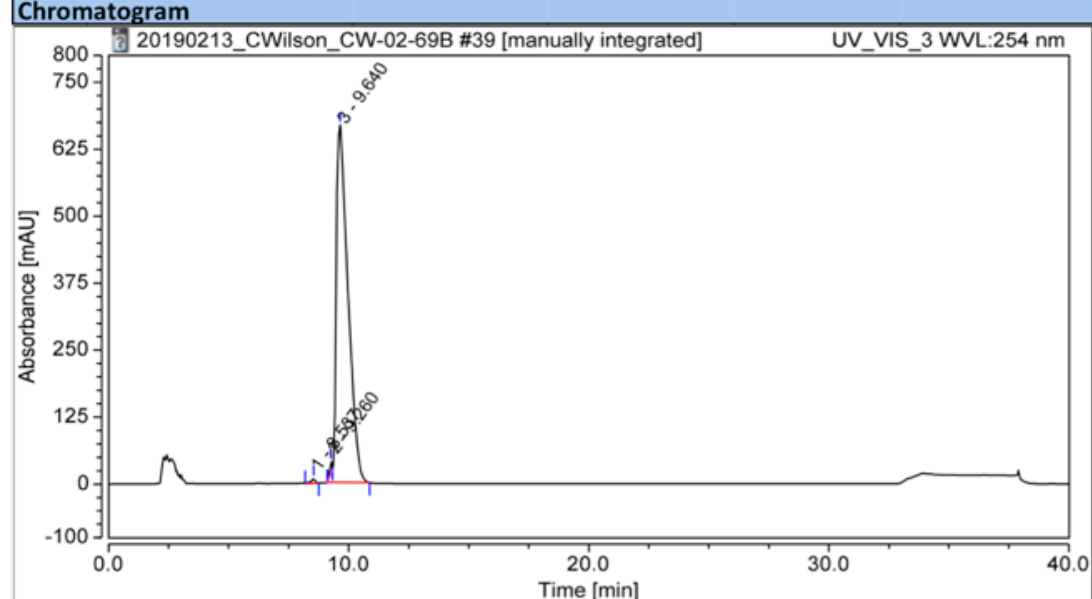

\begin{tabular}{|l|c|c|c|c|c|}
\hline \multicolumn{5}{|l|}{ Integration Results } \\
\hline No. & $\begin{array}{c}\text { Retention Time } \\
\text { min }\end{array}$ & $\begin{array}{c}\text { Area } \\
\text { mAU*min }\end{array}$ & $\begin{array}{c}\text { Height } \\
\text { mAU }\end{array}$ & $\begin{array}{c}\text { Relative Area } \\
\%\end{array}$ & $\begin{array}{c}\text { Relative Height } \\
\%\end{array}$ \\
\hline 1 & 8.537 & 1.663 & 8.473 & 0.44 & 1.21 \\
2 & 9.260 & 2.225 & 22.381 & 0.58 & 3.21 \\
3 & 9.640 & 377.021 & 667.116 & 98.98 & 95.58 \\
\hline \multicolumn{7}{|l|}{ Total: } & 380.909 & 697.970 & 100.00 & 100.00 \\
\hline
\end{tabular}

B)

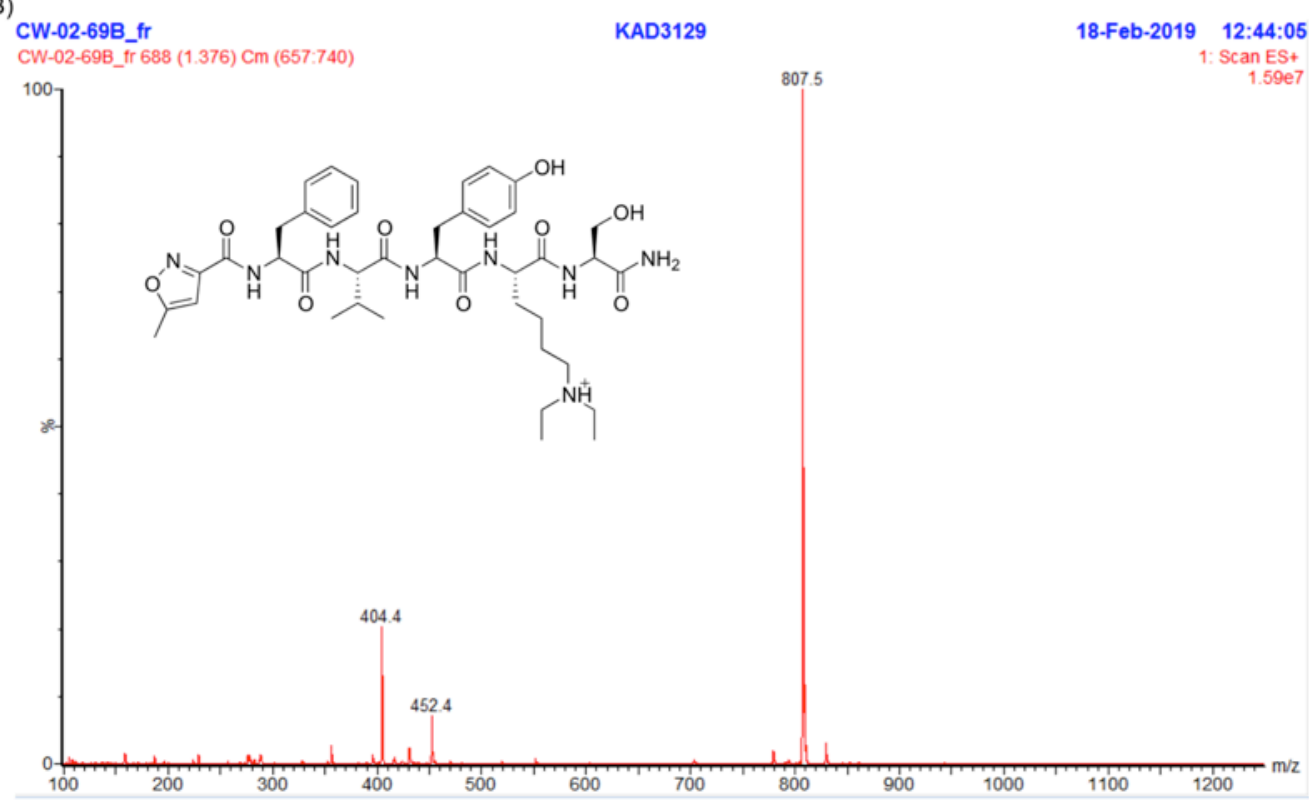

Supplementary Figure S9: Characterization data for compound 12. A) Analytical HPLC trace, B) Low resolution mass spectrum. LR-ESI-MS: $[\mathrm{m} / z]$ calcd. for: $\mathrm{C}_{41} \mathrm{H}_{59} \mathrm{~N}_{8} \mathrm{O}_{9}{ }^{+}: 807.44$; found: 807.5. 
A)

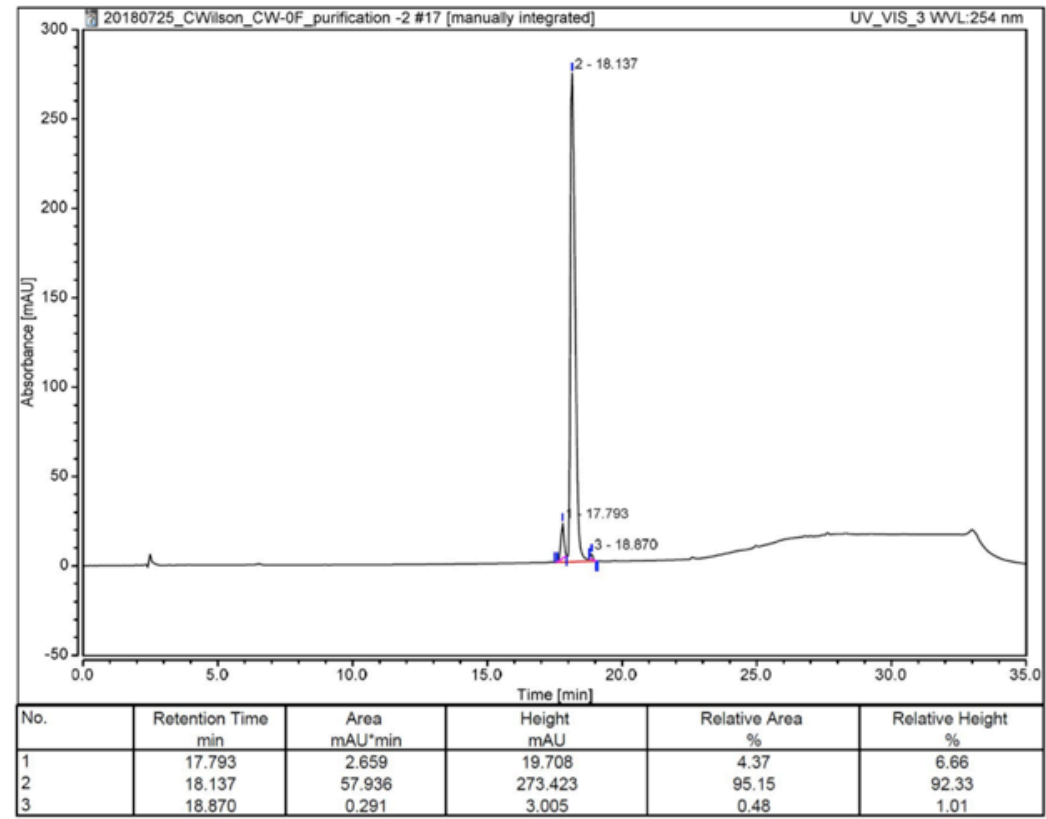

B)

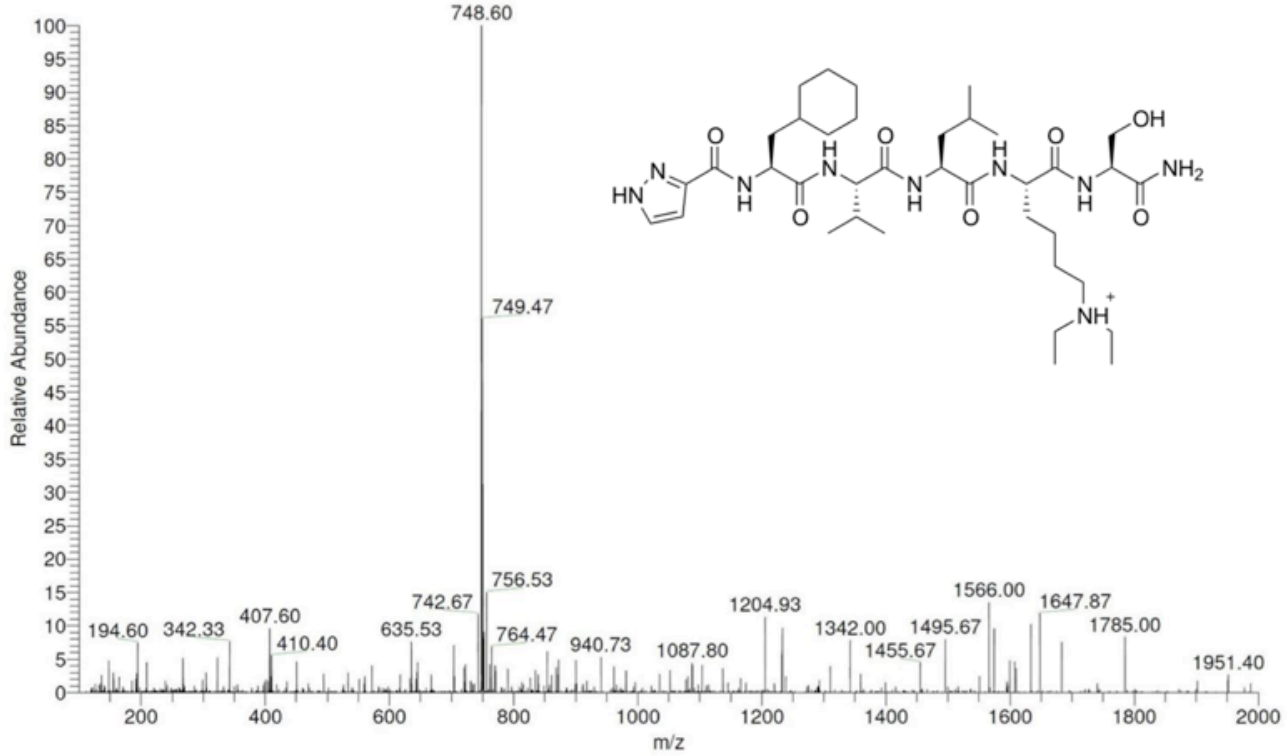

Supplementary Figure S10: Characterization data for compound 13. A) Analytical HPLC trace, B) Low resolution mass spectrum. LR-ESI-MS: $[\mathrm{m} / z]$ calcd. for: $\mathrm{C}_{37} \mathrm{H}_{66} \mathrm{~N}_{9} \mathrm{O}_{7}{ }^{+}$: 748.51 ; found: 748.60 . 
A)

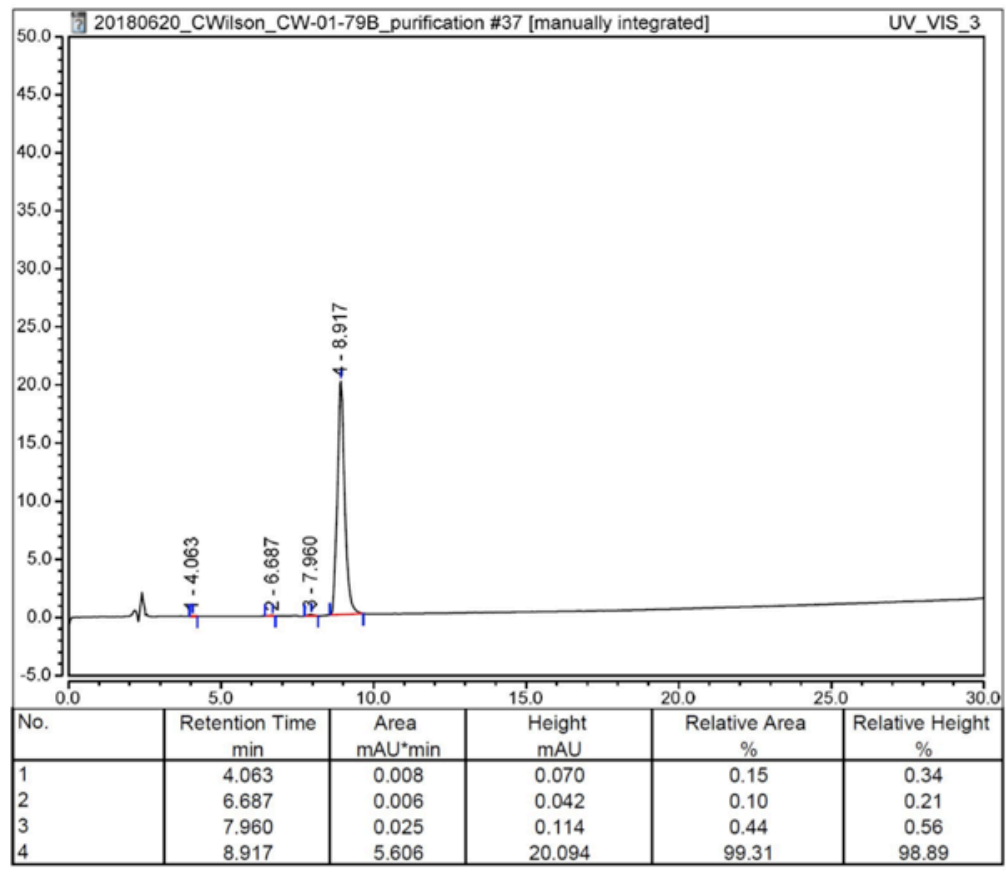

B)

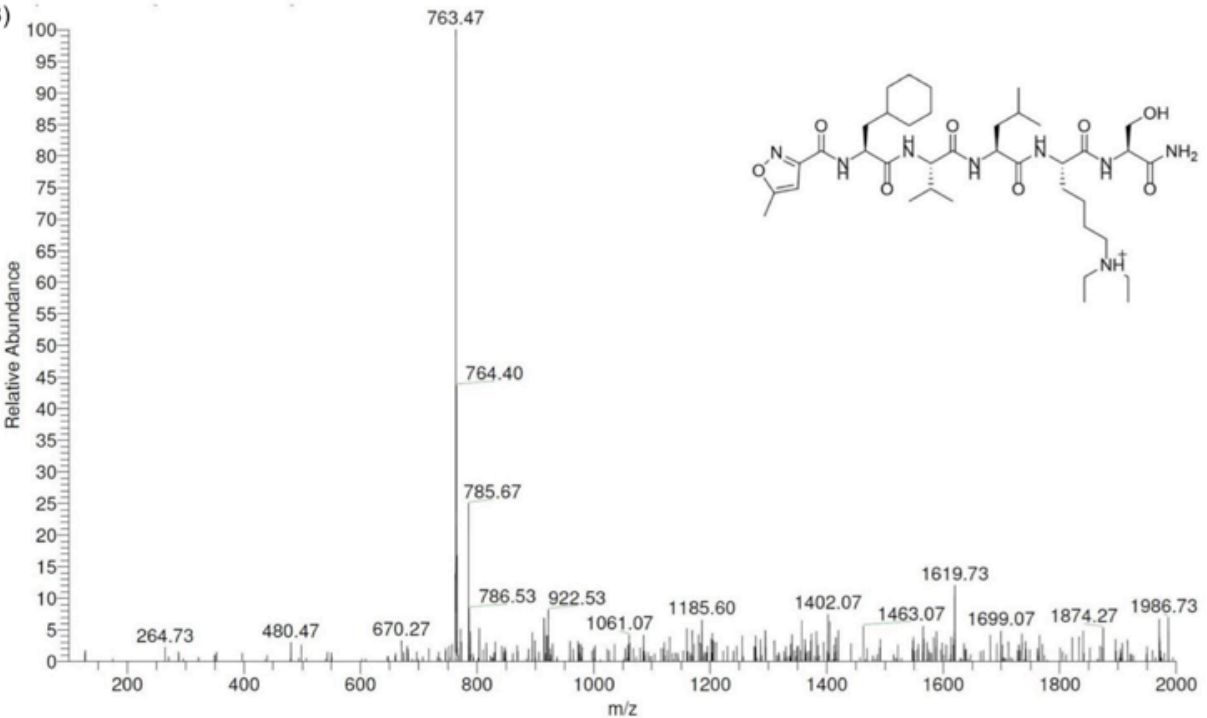

Supplementary Figure S11: Characterization data for compound 14. A) Analytical HPLC trace, B) Low resolution mass spectrum. LR-ESI-MS: $[\mathrm{m} / z]$ calcd. for: $\mathrm{C}_{38} \mathrm{H}_{67} \mathrm{~N}_{8} \mathrm{O}_{8}{ }^{+}: 763.51$; found: 763.47 . 
A)

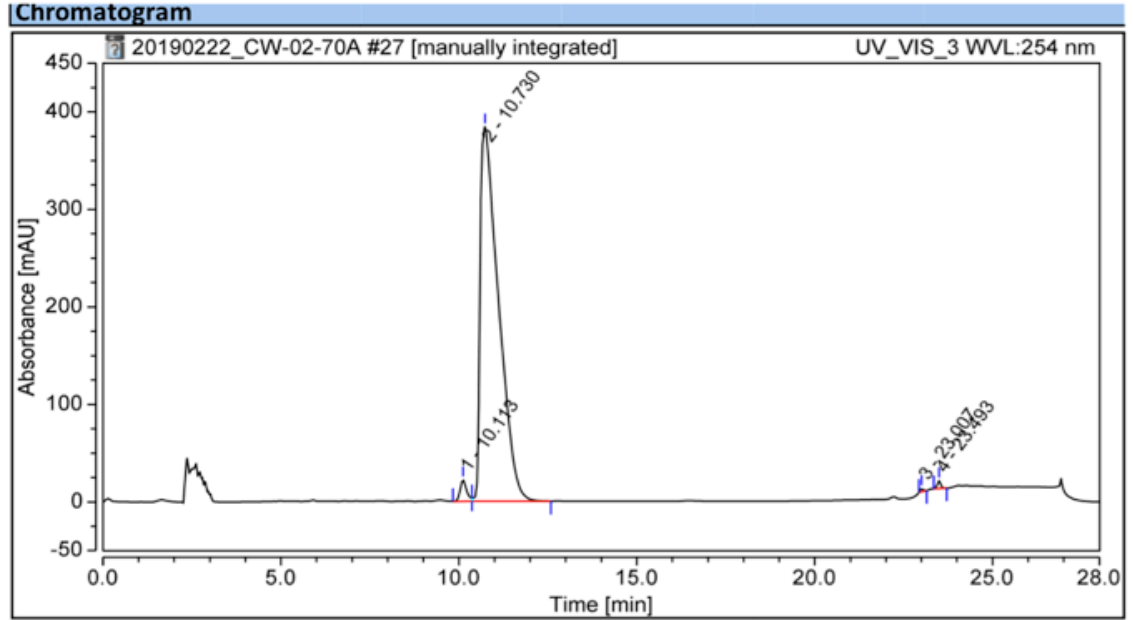

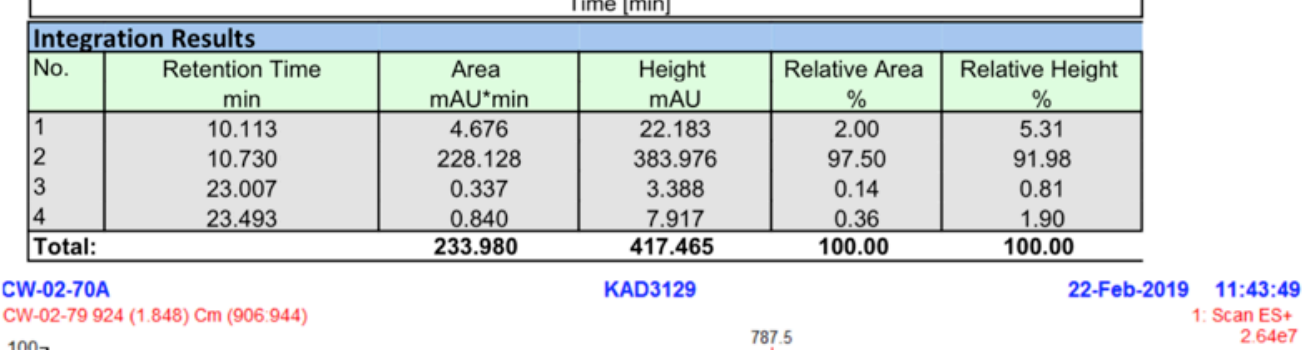

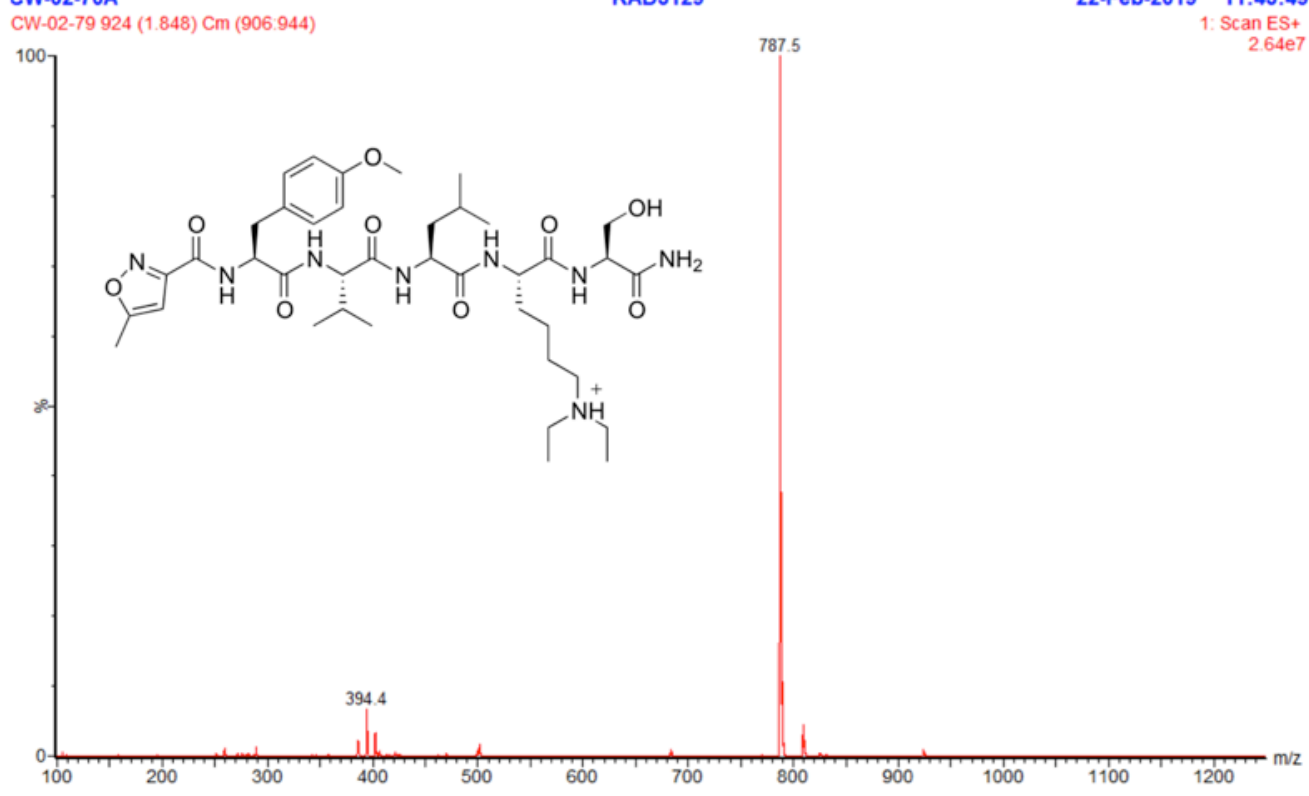

Supplementary Figure S12: Characterization data for compound 15. A) Analytical HPLC trace, B) Low resolution mass spectrum. LR-ESI-MS: $[m / z]$ calcd. for: $\mathrm{C}_{39} \mathrm{H}_{63} \mathrm{~N}_{8} \mathrm{O}_{9}{ }^{+}$: 787.47; found: 787.5 . 
A)

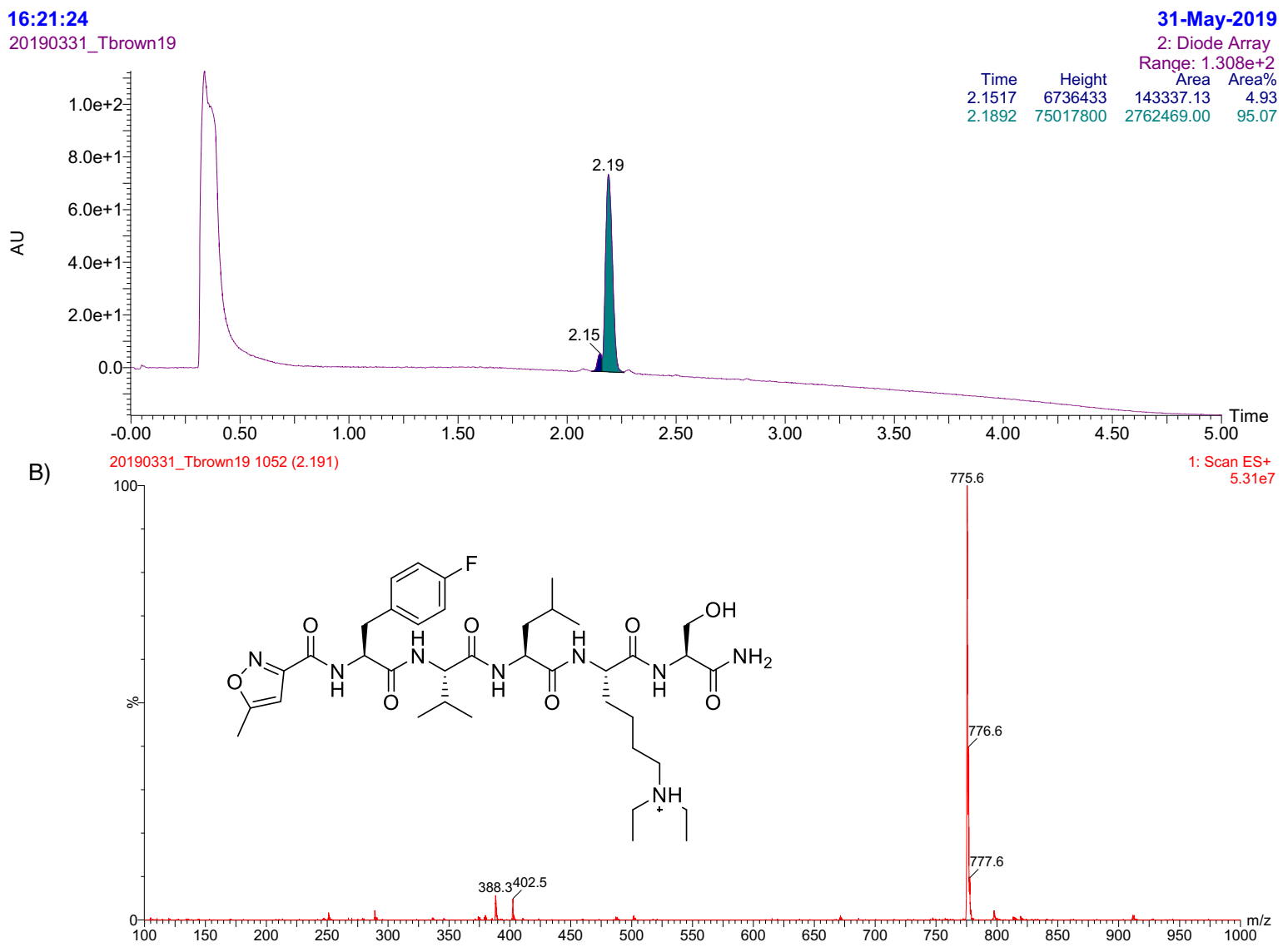

Supplementary Figure S13: Characterization data for compound 16. A) Analytical HPLC trace, B) Low resolution mass spectrum. LR-ESI-MS: $[\mathrm{m} / z]$ calcd. for: $\mathrm{C}_{38} \mathrm{H}_{60} \mathrm{FN}_{8} \mathrm{O}_{8}{ }^{+}$: 775.45 ; found: 775.6 
A)

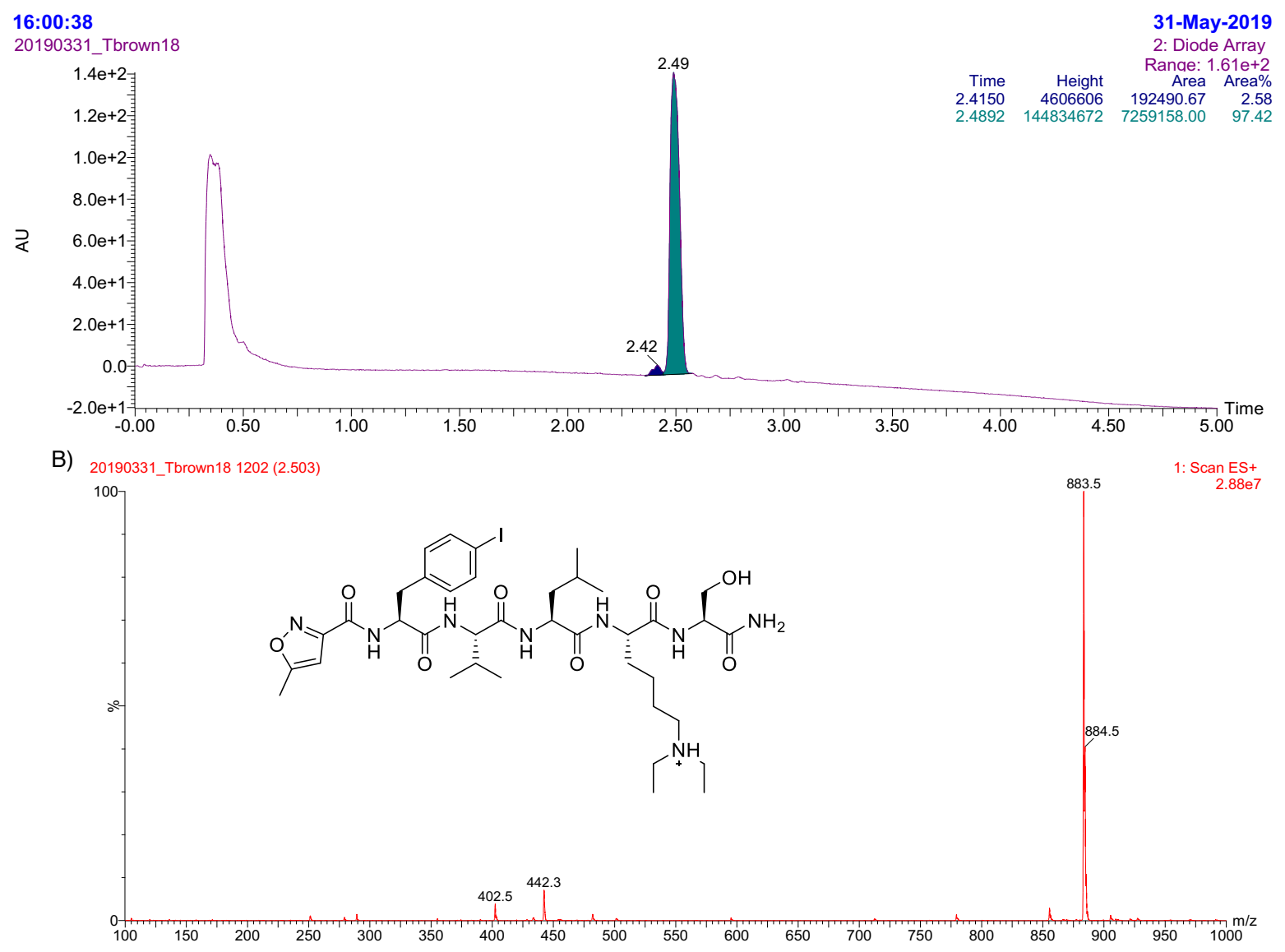

Supplementary Figure S14: Characterization data for compound 17. A) Analytical HPLC trace, B) Low resolution mass spectrum. LR-ESI-MS: $[\mathrm{m} / \mathrm{z}]$ calcd. for: $\mathrm{C}_{38} \mathrm{H}_{60} \mathrm{IN}_{8} \mathrm{O}_{8}{ }^{+}$: 883.36; found: 883.5 
A)

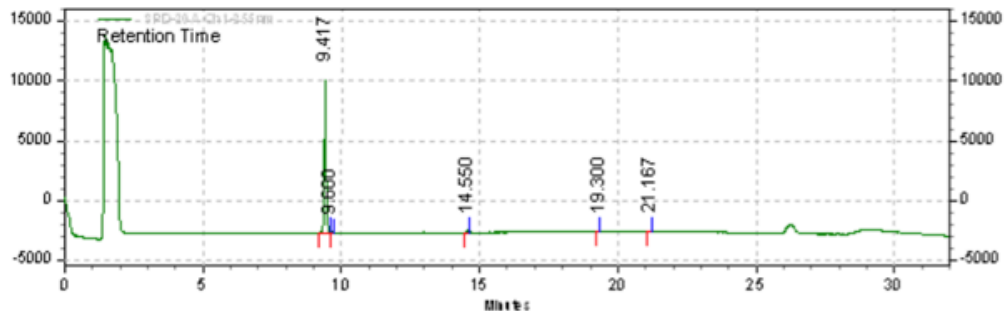

SPD-20A

Chl-255num

Results

\begin{tabular}{rrrrr} 
Retention Time & Area & Area \% & Height & Height \% \\
\hline 9.417 & 53942 & 97.45 & 12752 & 97.67 \\
9.600 & 3 & 0.01 & 0 & 0.00 \\
14.550 & 907 & 1.64 & 210 & 1.61 \\
19.300 & 225 & 0.41 & 55 & 0.42 \\
21.167 & 277 & 0.50 & 39 & 0.30
\end{tabular}

B)

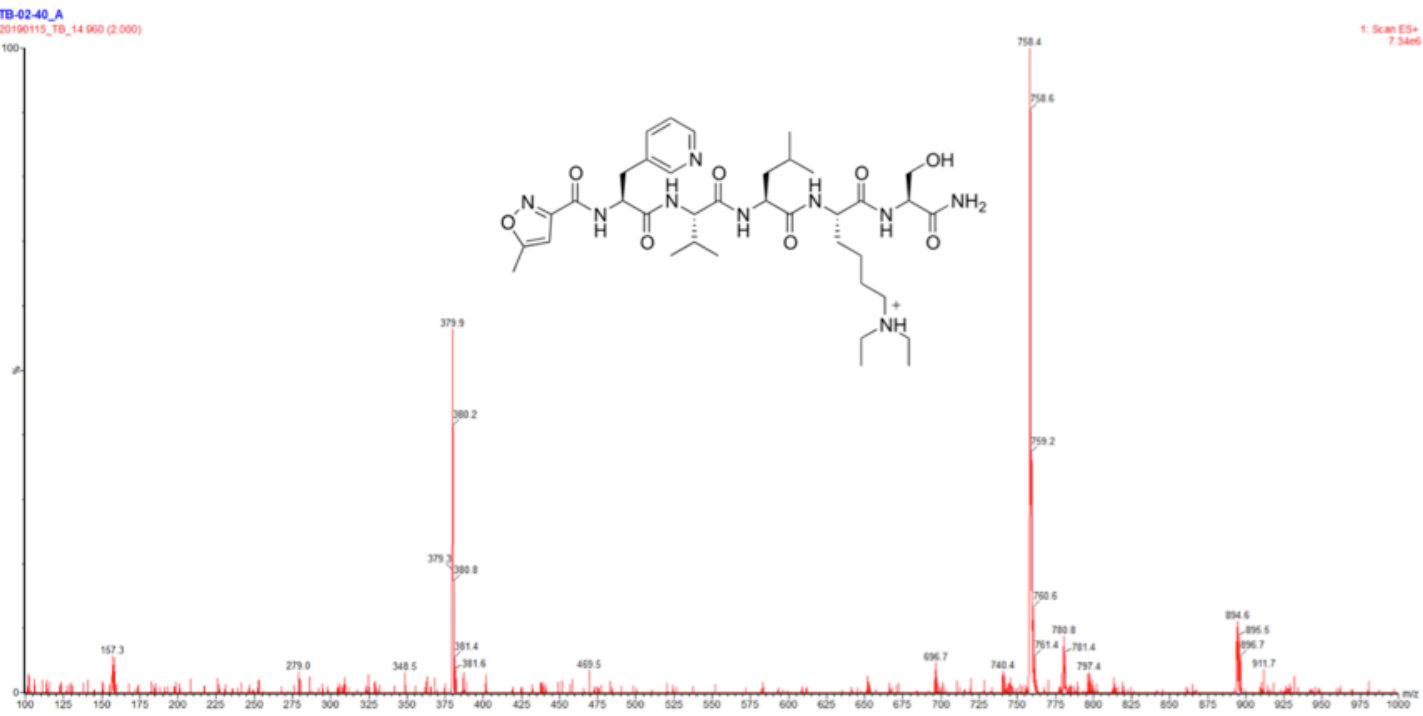

Supplementary Figure S15: Characterization data for compound 18. A) Analytical HPLC trace, B) Low resolution mass spectrum. LR-ESI-MS: $[\mathrm{m} / z]$ calcd. for: $\mathrm{C}_{37} \mathrm{H}_{60} \mathrm{~N}_{9} \mathrm{O}_{8}{ }^{+}: 758.46$; found: 758.4 . 
A)

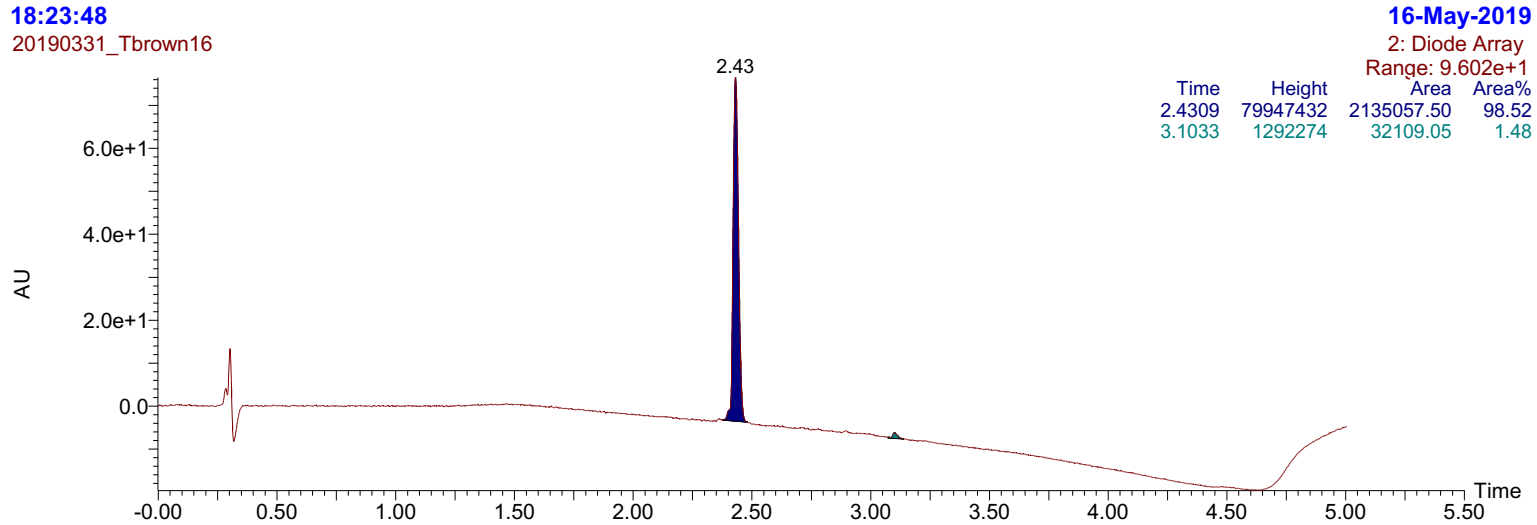

B) 20190331_Tbrown16 1168 (2.433)

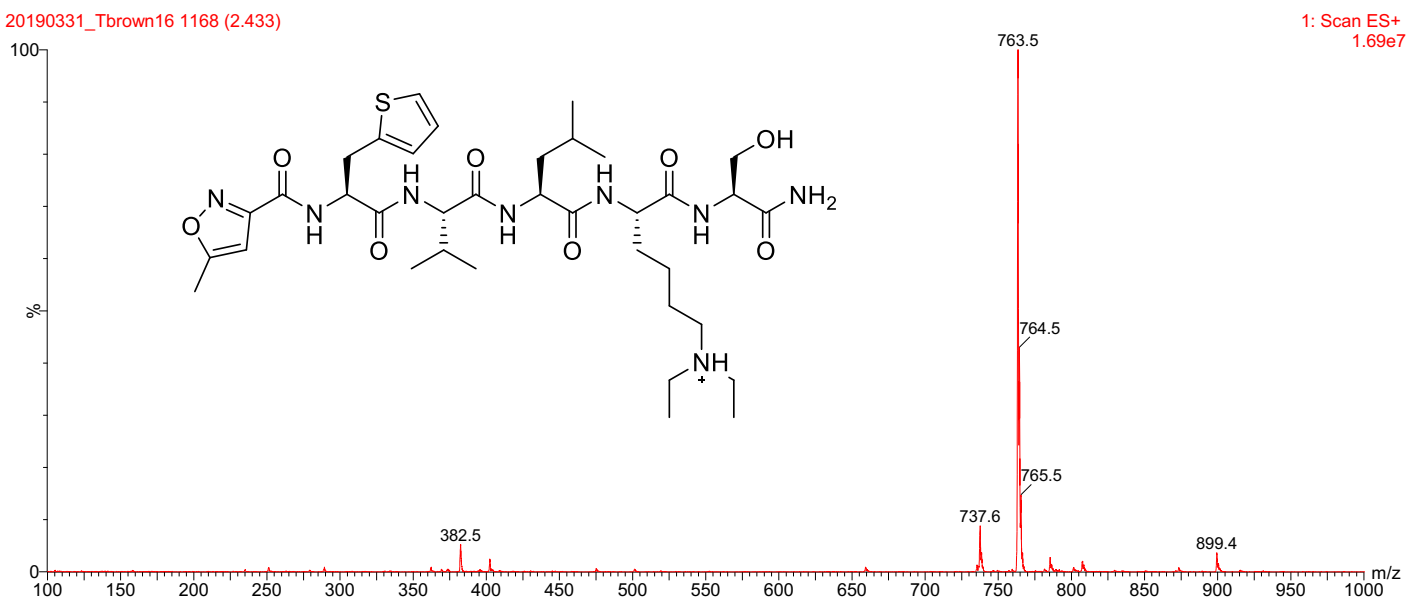

Supplementary Figure S16: Characterization data for compound 19. A) Analytical HPLC trace, B) Low resolution mass spectrum. LR-ESI-MS: $[\mathrm{m} / \mathrm{z}]$ calcd. for: $\mathrm{C}_{36} \mathrm{H}_{59} \mathrm{~N}_{8} \mathrm{O}_{8} \mathrm{~S}^{+}$: 763.42 ; found: 763.5 


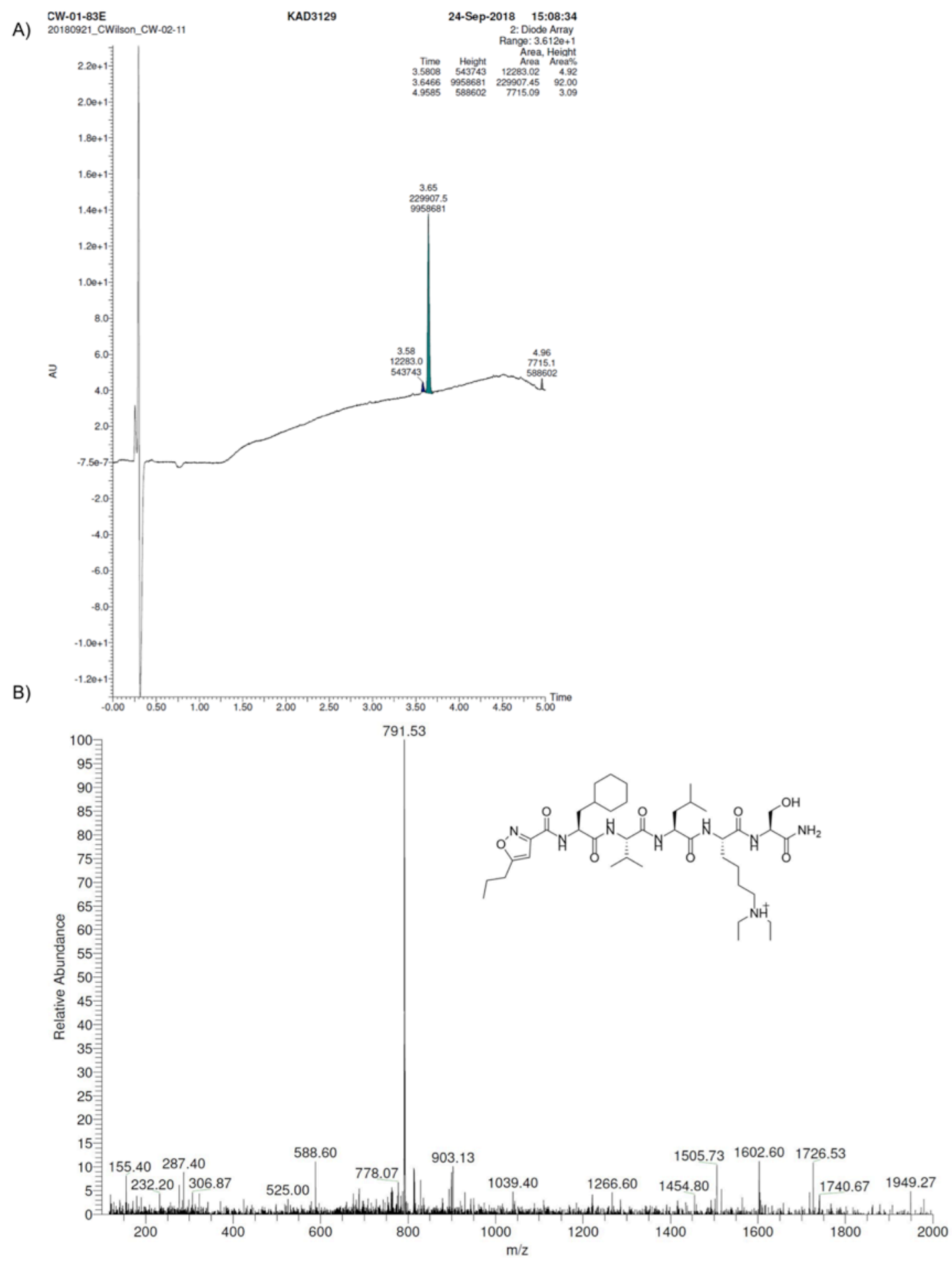

Supplementary Figure S17: Characterization data for compound 20. A) Analytical UPLC trace. Product peak is at 3.65 minutes with an area of $92 \%$. B) Low resolution mass spectrum. LR-ESIMS: [m/z] calcd. for: $\mathrm{C}_{40} \mathrm{H}_{71} \mathrm{~N}_{8} \mathrm{O}_{8}{ }^{+}$: 791.54; found: 791.53 . 
A)

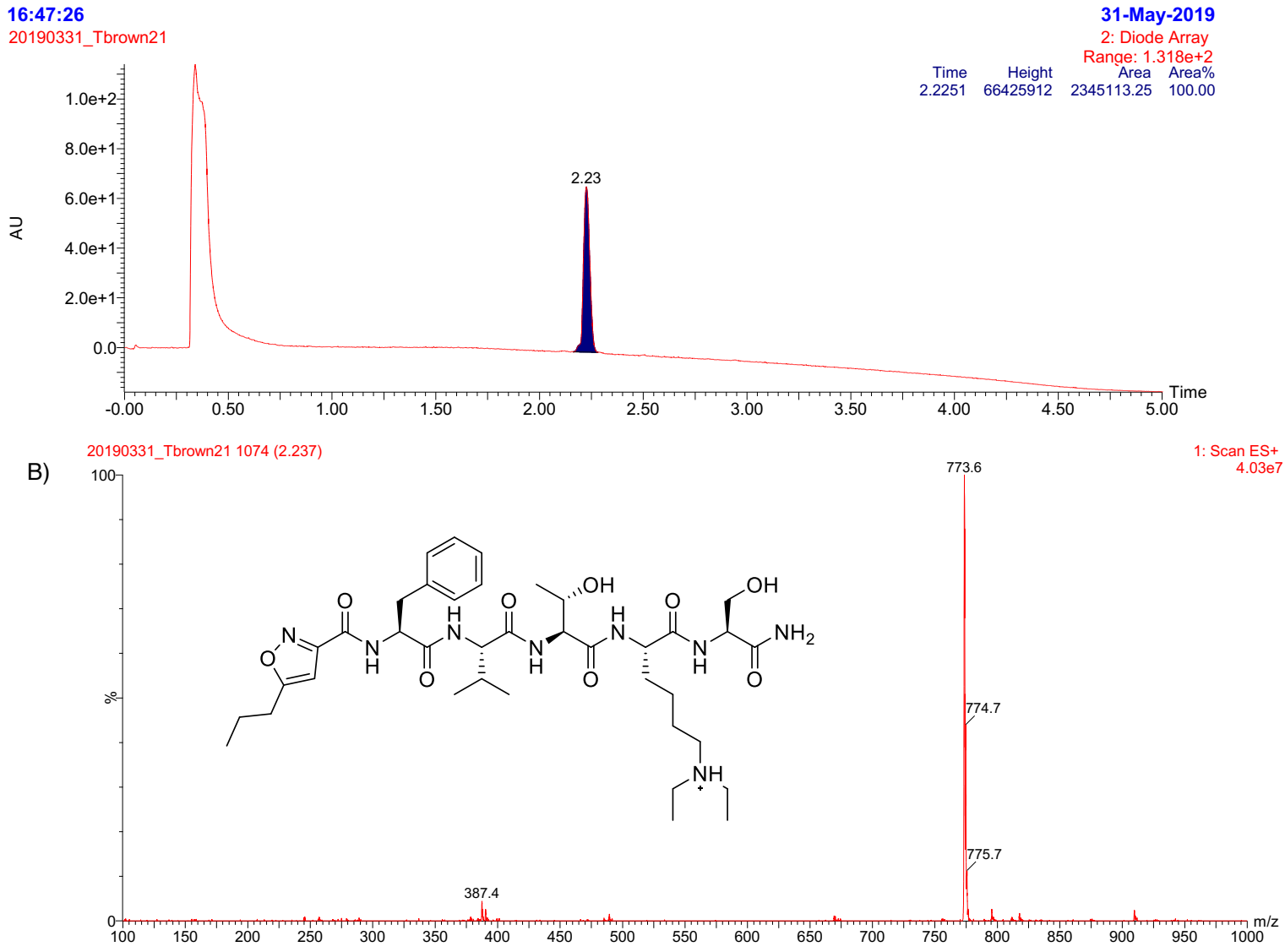

Supplementary Figure S18: Characterization data for compound 21. A) Analytical HPLC trace, B) Low resolution mass spectrum. LR-ESI-MS: $[m / z]$ calcd. for: $\mathrm{C}_{41} \mathrm{H}_{67} \mathrm{~N}_{8} \mathrm{O}_{8}{ }^{+}$: 773.46; found: 773.6 


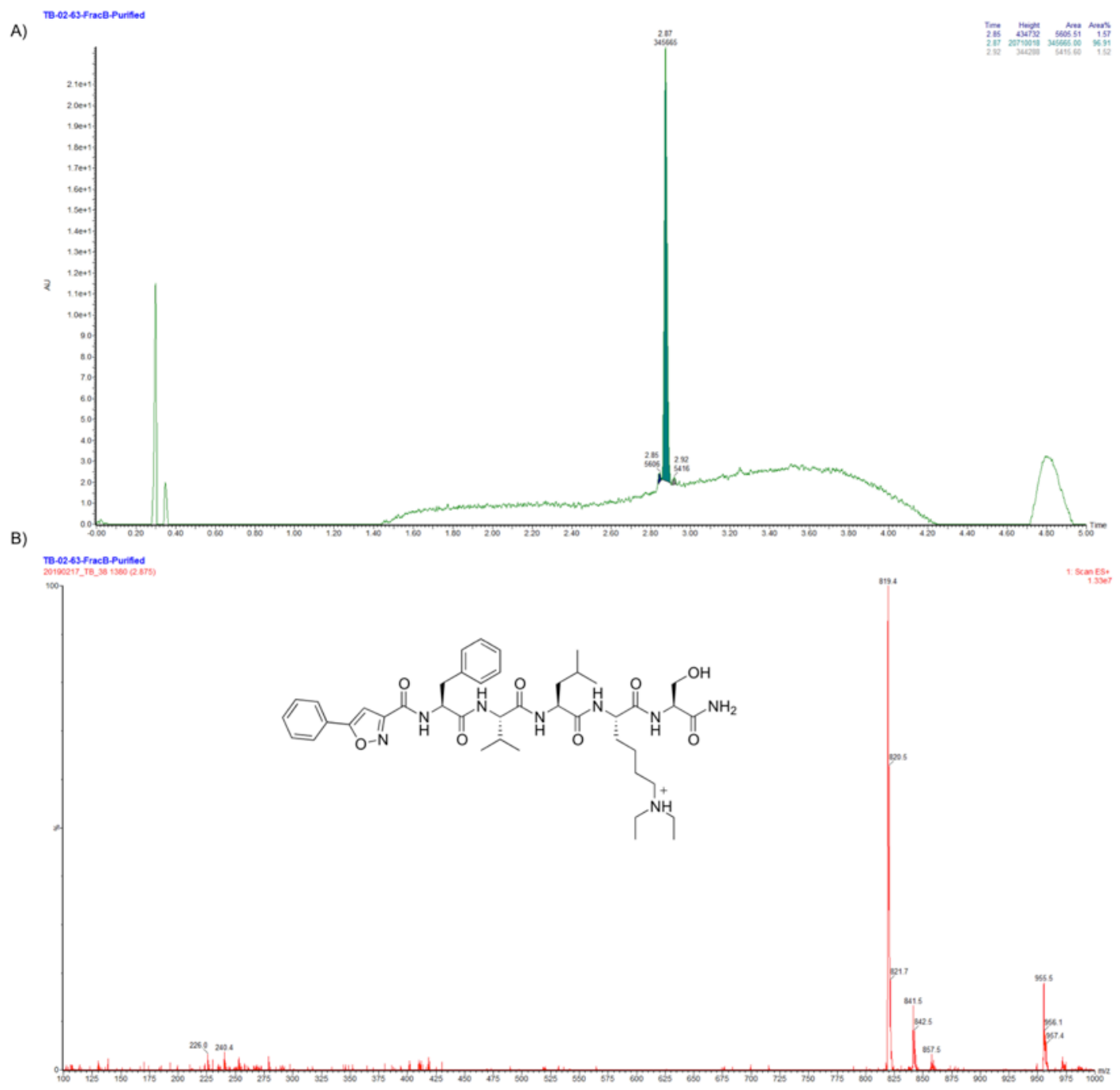

Supplementary Figure S19: Characterization data for compound 22. A) Analytical UPLC trace. Product peak is at 2.87 minutes with an area of 97\%. B) Low resolution mass spectrum. LR-ESIMS: $[m / z]$ calcd. for: $\mathrm{C}_{43} \mathrm{H}_{63} \mathrm{~N}_{8} \mathrm{O}_{8}{ }^{+}:$819.48; found: 819.4. 


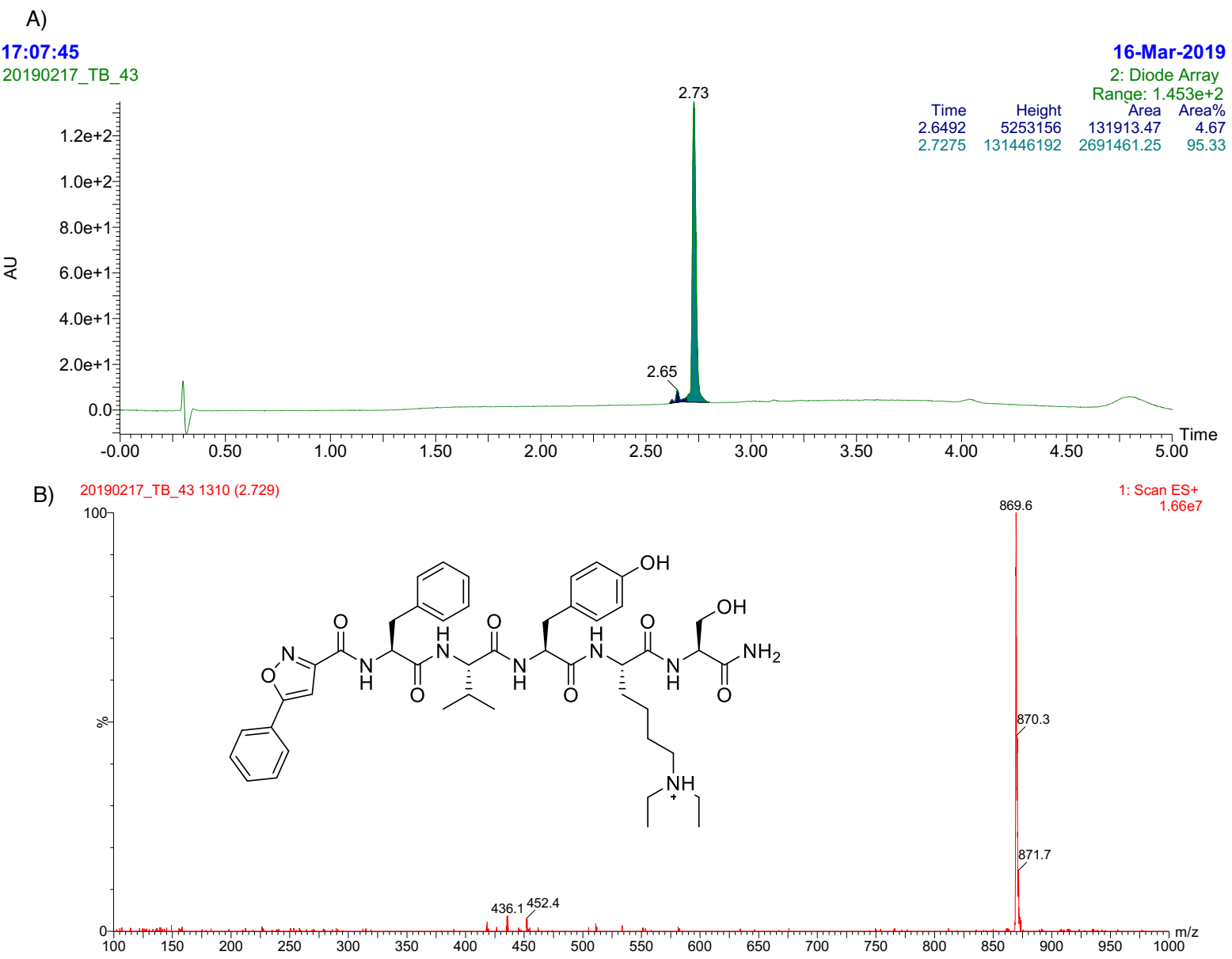

Supplementary Figure S20: Characterization data for compound 23. A) Analytical HPLC trace, B) Low resolution mass spectrum. LR-ESI-MS: $[m / z]$ calcd. for: $\mathrm{C}_{46} \mathrm{H}_{61} \mathrm{~N}_{8} \mathrm{O}_{9}{ }^{+}$: 869.46 ; found: 869.6 
A)

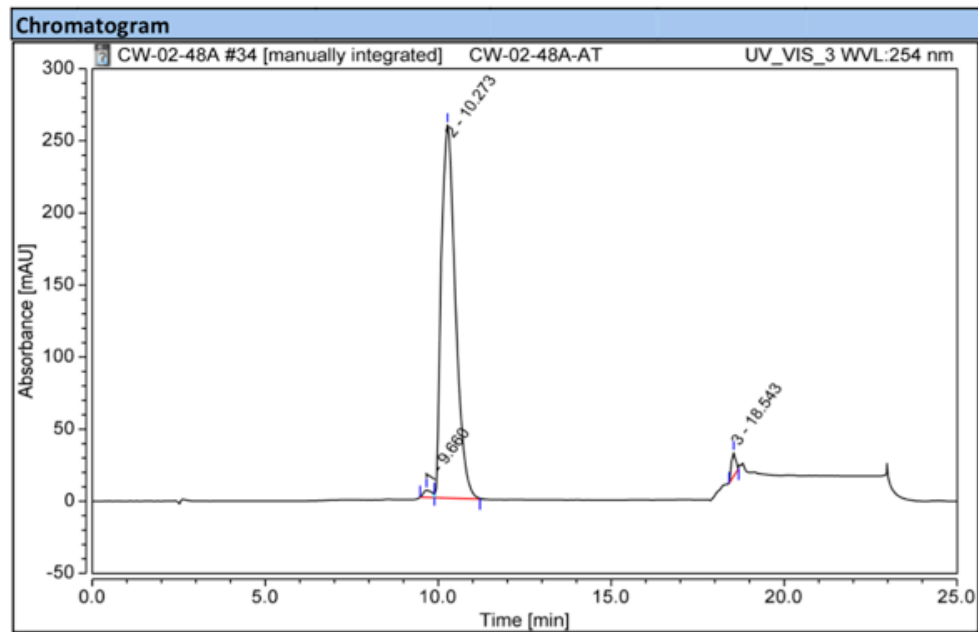

\begin{tabular}{|l|c|c|c|c|c|}
\hline \multicolumn{2}{|l|}{ Integration Results } \\
\hline No. & $\begin{array}{c}\text { Retention Time } \\
\text { min }\end{array}$ & $\begin{array}{c}\text { Area } \\
\text { mAU*min }\end{array}$ & $\begin{array}{c}\text { Height } \\
\text { mAU }\end{array}$ & $\begin{array}{c}\text { Relative Area } \\
\%\end{array}$ & $\begin{array}{c}\text { Relative Height } \\
\%\end{array}$ \\
\hline 1 & 9.660 & 1.378 & 4.891 & 1.10 & 1.75 \\
2 & 10.273 & 122.145 & 258.830 & 97.07 & 92.45 \\
3 & 18.543 & 2.306 & 16.255 & 1.83 & 5.81 \\
\hline Total: & 125.829 & $\mathbf{2 7 9 . 9 7 6}$ & $\mathbf{1 0 0 . 0 0}$ & $\mathbf{1 0 0 . 0 0}$ \\
\hline
\end{tabular}

B)
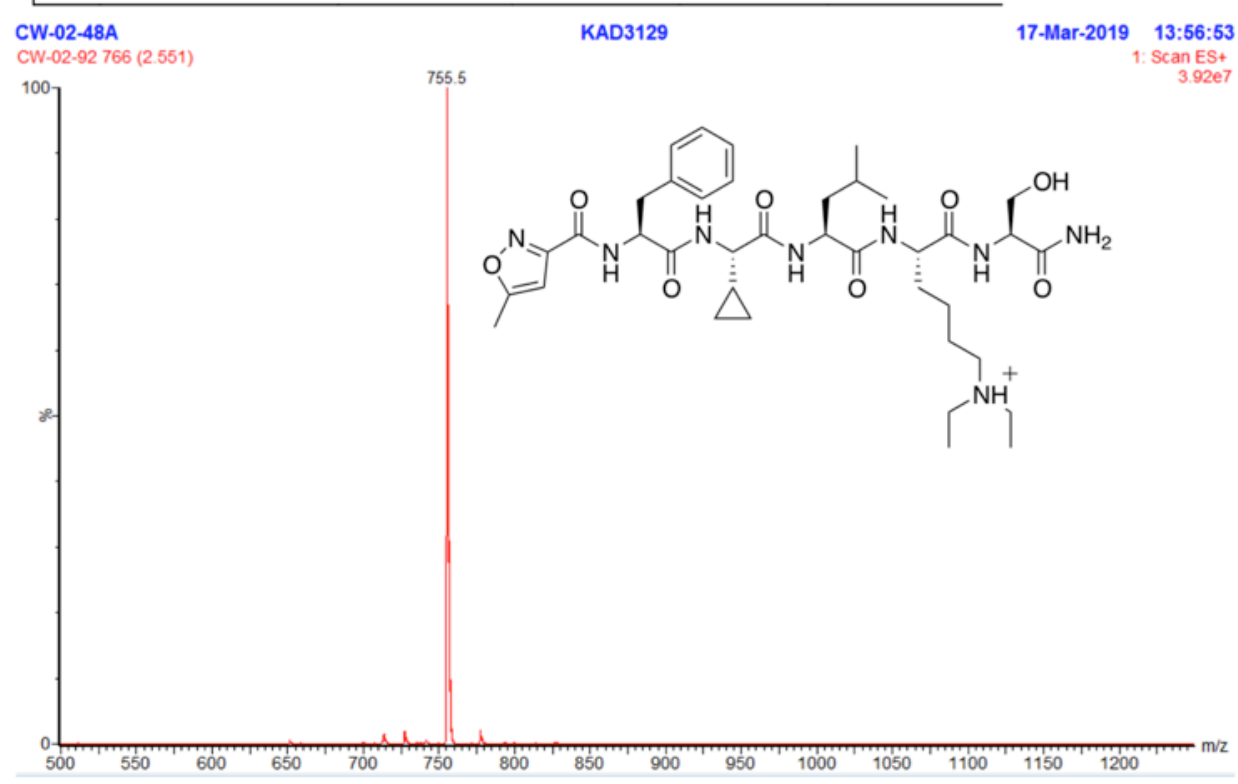

Supplementary Figure S21: Characterization data for compound 24. A) Analytical UPLC trace, B) Low resolution mass spectrum. LR-ESI-MS: $[\mathrm{m} / z]$ calcd. for: $\mathrm{C}_{38} \mathrm{H}_{59} \mathrm{~N}_{8} \mathrm{O}_{8}{ }^{+}: 755.45$; found: 755.5 . 
A)

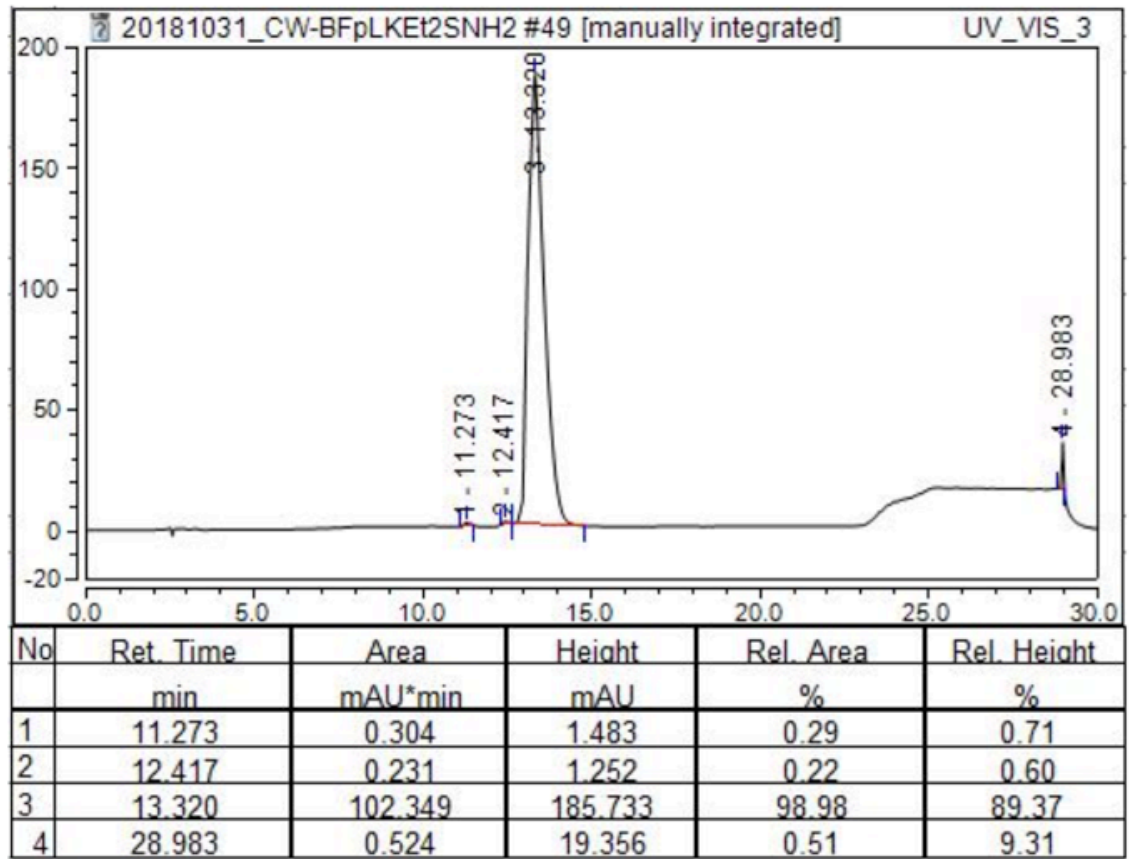

B)

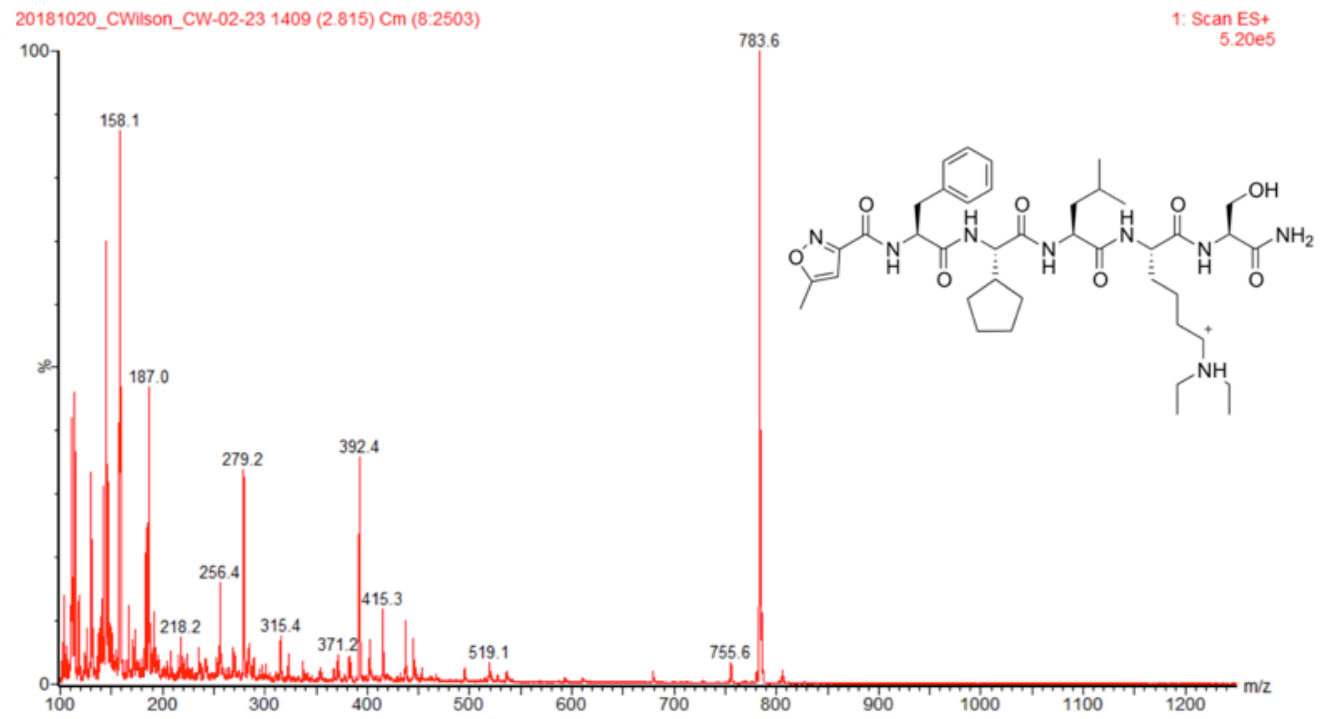

Supplementary Figure S22: Characterization data for compound 25. A) Analytical HPLC trace, B) Low resolution mass spectrum. LR-ESI-MS: $[\mathrm{m} / z]$ calcd. for: $\mathrm{C}_{40} \mathrm{H}_{63} \mathrm{~N}_{8} \mathrm{O}_{8}{ }^{+}: 783.48$; found: 783.6 . 
A) Chromatogram

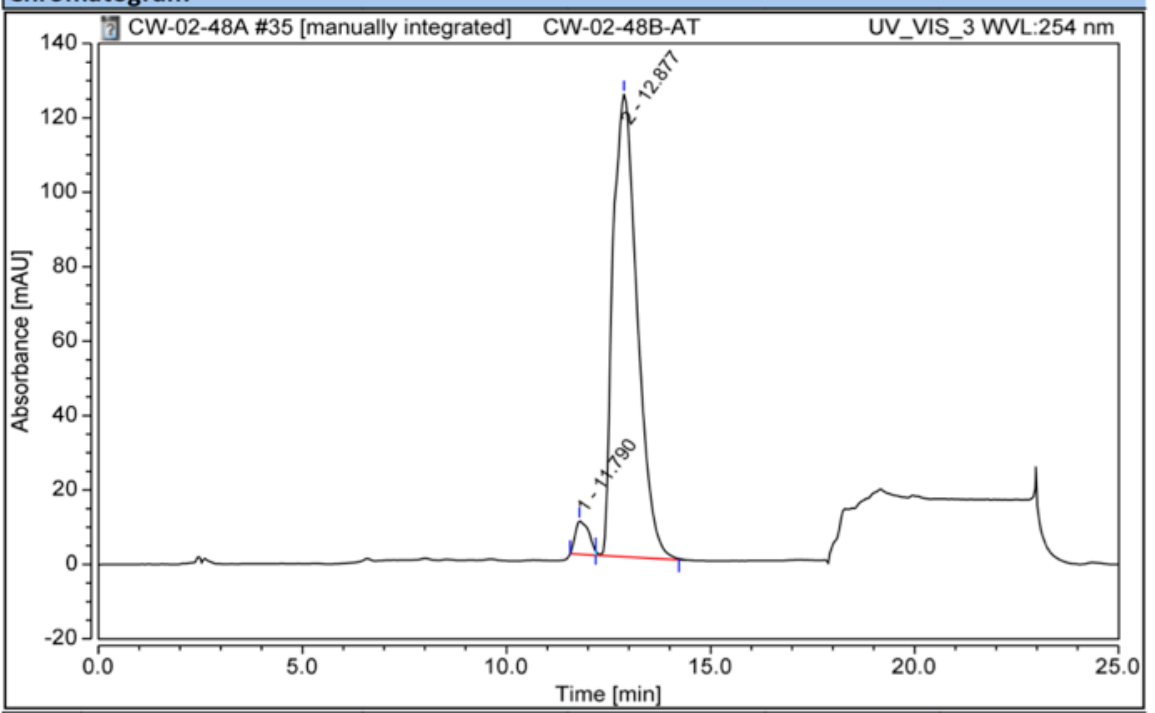

\begin{tabular}{|l|c|c|c|c|c|}
\hline \multicolumn{6}{|l|}{ Integration Results } \\
\hline No. & $\begin{array}{c}\text { Retention Time } \\
\text { min }\end{array}$ & $\begin{array}{c}\text { Area } \\
\text { mAU*min }\end{array}$ & $\begin{array}{c}\text { Height } \\
\text { mAU }\end{array}$ & $\begin{array}{c}\text { Relative Area } \\
\%\end{array}$ & $\begin{array}{c}\text { Relative Height } \\
\%\end{array}$ \\
\hline 1 & 11.790 & 3.475 & 8.964 & 3.90 & 6.73 \\
2 & 12.877 & 85.614 & 124.304 & 96.10 & 93.27 \\
\hline \multicolumn{7}{|l|}{ Total: } & $\mathbf{8 9 . 0 8 9}$ & $\mathbf{1 3 3 . 2 6 8}$ & 100.00 & 100.00 \\
\hline
\end{tabular}

B)

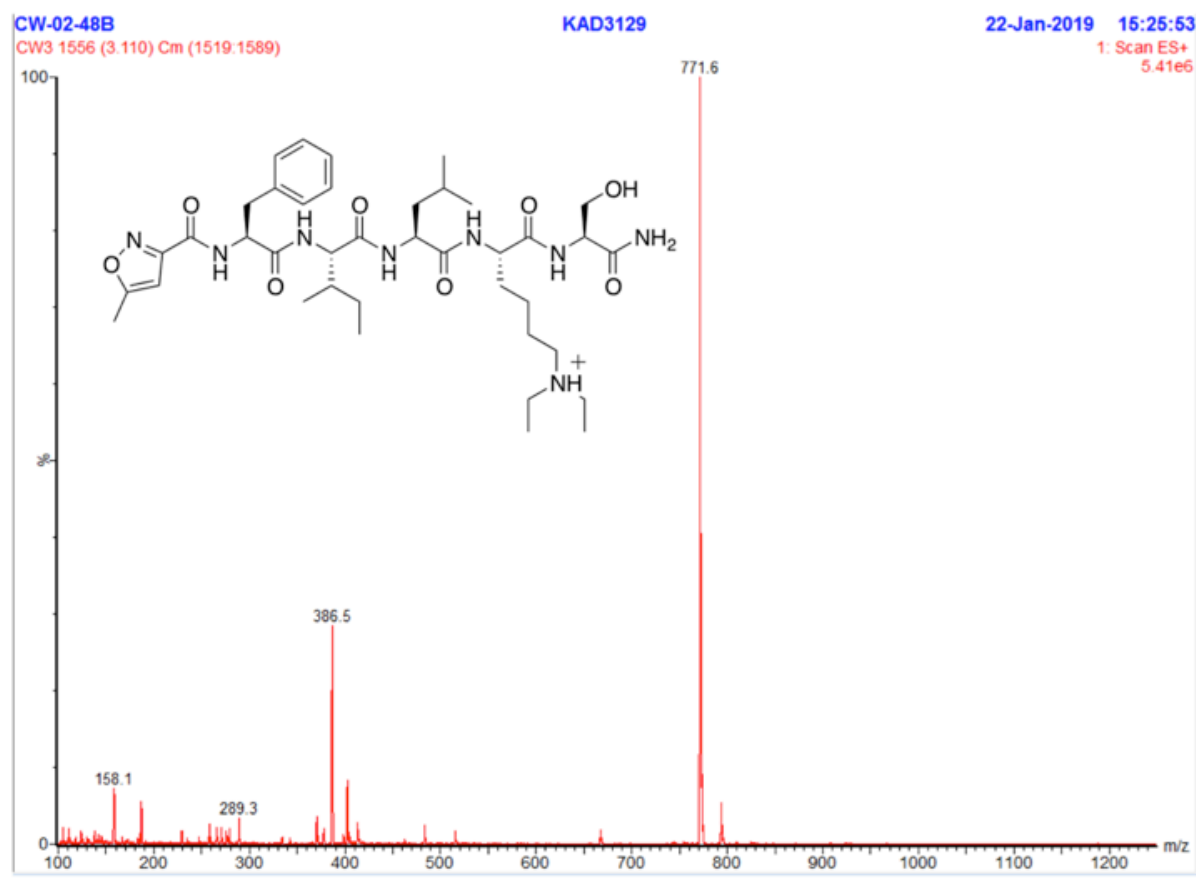

Supplementary Figure S23: Characterization data for compound 26. A) Analytical HPLC trace, B) Low resolution mass spectrum. LR-ESI-MS: $[\mathrm{m} / \mathrm{z}]$ calcd. for: $\mathrm{C}_{39} \mathrm{H}_{63} \mathrm{~N}_{8} \mathrm{O}_{8}{ }^{+}: 771.48$; found: 


\section{Protein expression and purification}

E. coli pellets were thawed overnight and sequentially lysed via a 30 minute incubation with a $\mathrm{CHAPS} / \mathrm{dH}_{2} \mathrm{O}$ solution (Biobasic) and sonication. After centrifugation to remove cell debris, the purification procedure involved two chromatographic steps. First, an affinity chromatographic step on a nickel-nitrilotriacetic acid chelating column (Qiagen) followed by a gel filtration step using an FPLC outfitted with a Hiload 16/600 Superdex 75 pg size exclusion column (GE). The FPLC step performed a buffer exchange into our 'minimal' fluorescence polarization (FP) buffer consisting of $20 \mathrm{mM}$ Tris $\mathrm{HCl}, 250 \mathrm{mM} \mathrm{NaCl}$, and $0.01 \%$ Tween-20. Purified protein was then concentrated to the desired volume using Amicon centrifugal filter units. Proteins were flash frozen and stored at $-80^{\circ} \mathrm{C}$ while purity was assessed by SDS page. His tags were cleaved off overnight after the nickel column step using our in-house TEV protease.

\section{Fluorescence polarization methods}

\section{Direct FP method}

Direct FP was done by titration of CBX proteins into FITC-labelled probes. Binding assays were performed in black 384 well plates with optical bottoms at a total volume of $50 \mu \mathrm{L}$ per well. All solutions were dissolved in 'full' FP buffer made from the addition of DTT, PMSF and benzamidine ( $1 \mathrm{mM}$ final concentration) into 'minimal' FP buffer (20 mM Tris HCl, $250 \mathrm{mM}$ $\mathrm{NaCl}, 0.01 \%$ Tween-20). A solution containing $100 \mathrm{nM}$ of the labelled probe and a varying concentration of the CBX protein in question, was titrated into wells containing a solution with a constant concentration (100 nM) of FITC-labelled probe. $100 \mu \mathrm{L}$ of the CBX-containing solution was added to well 19. Wells $2-18$ contained $50 \mu \mathrm{L}$ of the FITC-probe only solution. $50 \mu \mathrm{L}$ of the well 19 solution was added to well 18 and mixed 4 times. These dilutions continued until well 3, whereupon $50 \mu \mathrm{L}$ was discarded after mixing, leaving the total volume of all wells at $50 \mu \mathrm{L}$. Well 1 contained only full FP buffer and well 2 contained only the FITC-probe in full FP buffer. The maximum concentrations used for each CBX protein varied $(0.001 \mu \mathrm{M}-600 \mu \mathrm{M})$ based on availability and solubility of the protein at high concentration, as well as the expected strength of the FITC-probe being tested. Assays were performed in triplicate or duplicate depending on CBX protein availability (noted in raw data). After removing any bubbles with a needle, plates were incubated in the dark at RT for 15 minutes and then read with a SpectraMax M5 plate reader (Molecular Devices). Raw data for perpendicular (P) and parallel (S) light was collected 
with SoftMax Pro software using a fluorescence polarization endpoint read. 100 reads were made and averaged per well with 5 second automix before reading and medium PMT sensitivity. Excitation was $450 \mathrm{~nm}$ for FITC and emission was $530 \mathrm{~nm}$ (with a $515 \mathrm{~nm}$ cut-off).

\section{Analysis of direct FP data}

The background of the blank buffer was subtracted from the parallel and perpendicular intensities of emission. Average values for parallel and perpendicular emission intensities (minus the average values for buffer control wells) were determined for sets of replicates using Microsoft Excel and millipolarization units $(\mathrm{mP})$ were calculated using the formula ((Parallel Perpendicular $) /($ Parallel + Perpendicular $))^{*} 1000$. Data were then plotted using Graphpad Prism 8 and fitted using a 'one-site' total binding model. The mathematical model for the fit was described by the equation $\mathrm{y}=(\operatorname{Bmax} \times \mathrm{x}) /\left(\mathrm{K}_{\mathrm{d}}+\mathrm{x}\right)+\mathrm{NS} \times \mathrm{x}+$ background $(\mathrm{Bmax}=$ maximum specific binding, NS = slope of nonspecific binding and background $=$ amount of nonspecific binding with no added labeled ligand). For certain assays, some of the data points (0 to 5 points) representing the highest $\mathrm{CBX}$ concentrations had to be excluded due to abnormally high $\mathrm{mP}$ values, likely arising from protein aggregation.

\section{Competitive FP method}

Competitive FP analysis of 1 and 4 binding to $\mathrm{CBX} 6 / 7 / 8$ was used to determine $\mathrm{IC}_{50}$ values as with compound $\mathbf{2}$ as the competitive binding probe. Compound $\mathbf{2}$ and peptide inhibitors were dissolved in DMSO and then diluted into FP buffer. DMSO was kept below $2.5 \%$ for the majority of the assays, with the exception of 6, 8, 22 and 23 (5\%DMSO). The assay was carried out in NUNC black 96-well plates and 'full' FP buffer was used as described above (20 mM Tris- $\mathrm{HCl}, \mathrm{pH}=8.0,250 \mathrm{mM} \mathrm{NaCl}, 1 \mathrm{mM}$ DTT, $1 \mathrm{mM}$ benzamidine, $1 \mathrm{mM}$ PMSF, 0.01\% Tween). Two solutions were used, one containing protein, FITC probe 2, and peptide inhibitor and another with just the protein and FITC probe $\mathbf{2}$. Probe $\mathbf{2}$ was at a constant concentration of $100 \mathrm{nM}$ in both solutions. Each CBX protein was at a constant concentration dependent on the $\mathrm{K}_{\mathrm{d}}$ of the protein to probe 2 . $\mathrm{CBX} 1$ was used at $10 \mu \mathrm{M}, \mathrm{CBX} 6$ at $1 \mu \mathrm{M}, \mathrm{CBX} 7$ at $0.4 \mu \mathrm{M}$ and CBX8 at $4 \mu \mathrm{M}$. Inhibitor concentrations varied between $0-500 \mu \mathrm{M}$. All wells in the plate were made to final volume of $100 \mu \mathrm{L} .200 \mu \mathrm{L}$ of the inhibitor-containing solution was added to well 12. Wells $2-11$ contained $100 \mu \mathrm{L}$ of the CBX and 2 only solution. $100 \mu \mathrm{L}$ of the well 12 solution 
was added to well 11 and mixed. These dilutions continued to well 3, whereupon $100 \mu \mathrm{L}$ was discarded after mixing, leaving the total volume of all wells at $100 \mu \mathrm{L}$. Well 1 contained only full FP buffer and well 2 contained only CBX protein and 2 in full FP buffer. Following serial dilution of the inhibitor across the plate, plates were incubated for 15 minutes in the dark and then were read with Cytation 5 cell imaging and multi-mode reader plate reader. A polarized filter cube was used with filters for excitation at $485 \mathrm{~nm}$ and emission at $520 \mathrm{~nm}$ (with a cut-off of $510 \mathrm{~nm}$ ). All plates were read at $28^{\circ} \mathrm{C}$.

\section{Analysis of competitive FP data}

The background of the blank buffer was subtracted from the parallel and perpendicular intensities of emission. Values were graphed using Graphpad Prism 8 and fitted using a sigmoidal curve function from which $\mathrm{IC}_{50}$ values were extrapolated. The equation used is described as follows: $\mathrm{y}=$ bottom $+($ top-bottom $) /\left(1+10^{\wedge}\left(\mathrm{X}-\log I \mathrm{C}_{50}\right)\right)$. Errors are reported as 95\% confidence intervals. Experiments were done in duplicate or triplicate and this is indicated in the raw data shown. 
Fluorescence polarization data

A)

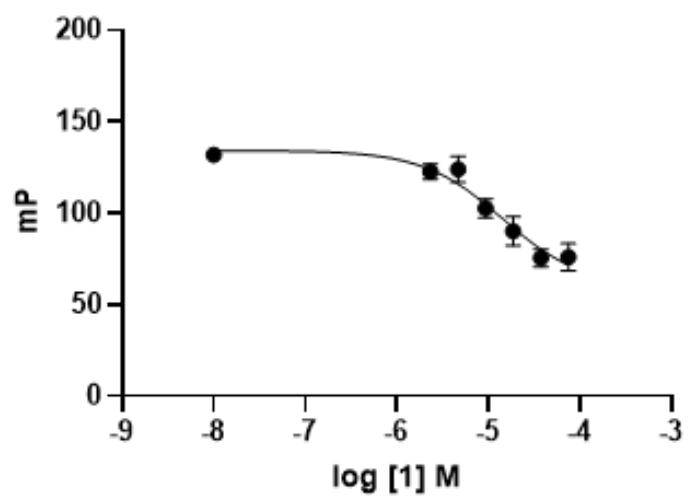

C)

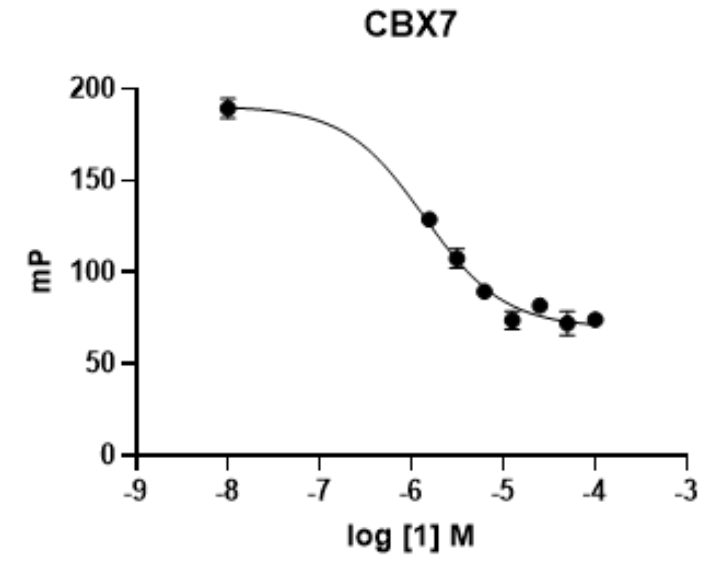

B)

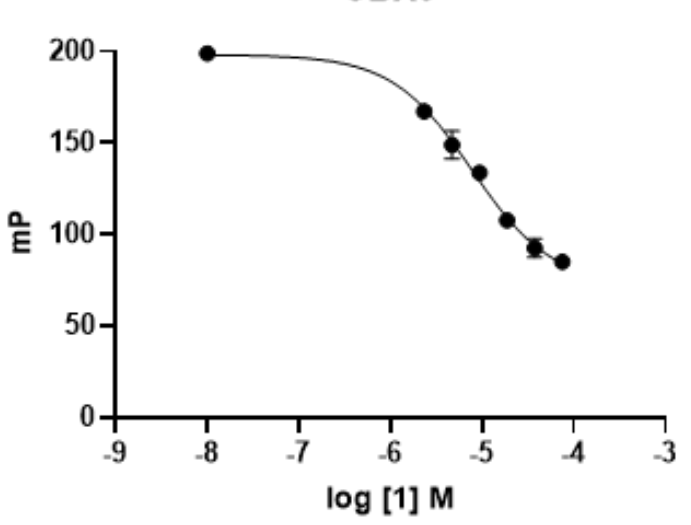

D)

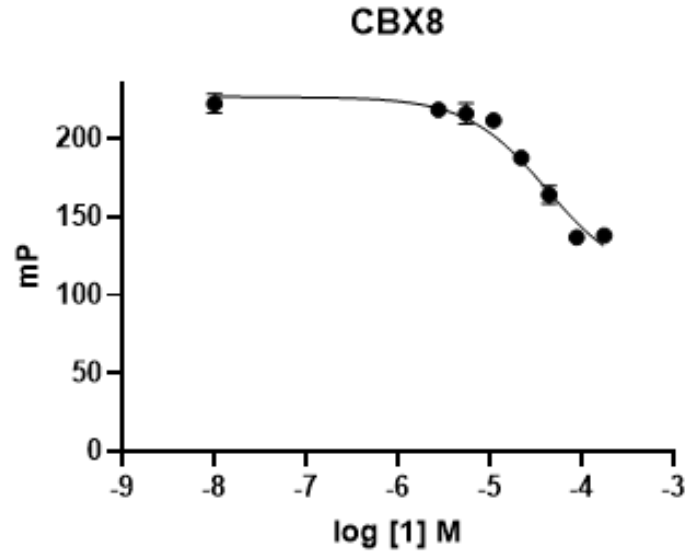

Supplementary Figure S24: Competitive fluorescence polarization data of compound 1 with CBX proteins preformed in duplicate. A) $\mathrm{CBX} 1\left(\log \mathrm{IC}_{50}=-4.845 \mathrm{M}, 95 \% \mathrm{CI}-5.137 \mathrm{M}\right.$ to $\left.\left.4.510 \mathrm{M}, \mathrm{R}^{2}=0.928\right), \mathrm{B}\right) \mathrm{CBX} 6\left(\log \mathrm{IC}_{50}=-5.113 \mathrm{M}, 95 \% \mathrm{CI}-5.222 \mathrm{M}\right.$ to $-5.00 \mathrm{M}, \mathrm{R}^{2}=$ 0.990), C) $\mathrm{CBX} 7\left(\log \mathrm{IC}_{50}=-5.862 \mathrm{M}, 95 \% \mathrm{CI}-5.983 \mathrm{M}\right.$ to $\left.\left.-5.746 \mathrm{M}, \mathrm{R}^{2}=0.983\right), \mathrm{D}\right) \mathrm{CBX} 8$ $\left(\log \mathrm{IC}_{50}=-4.385 \mathrm{M} 95 \% \mathrm{CI}-4.593 \mathrm{M}\right.$ to $\left.-4.162 \mathrm{M}, \mathrm{R}^{2}=0.967\right)$. Error bars reported as asymmetrical $95 \%$ confidence intervals. 
A)

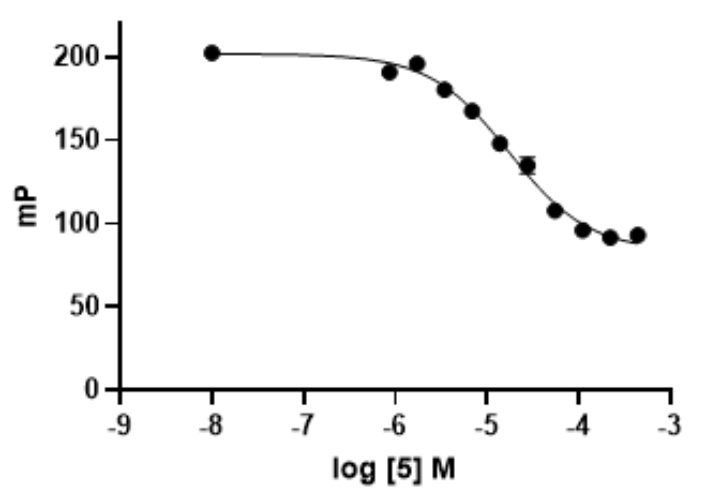

C)

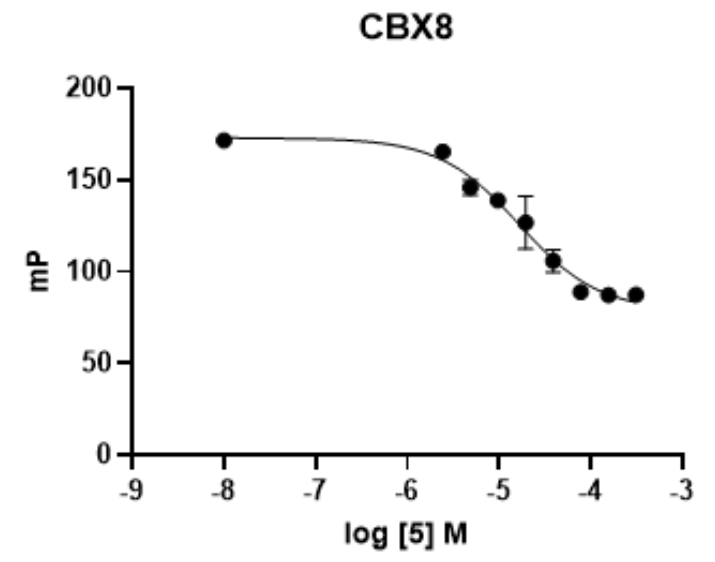

B)

CBX7

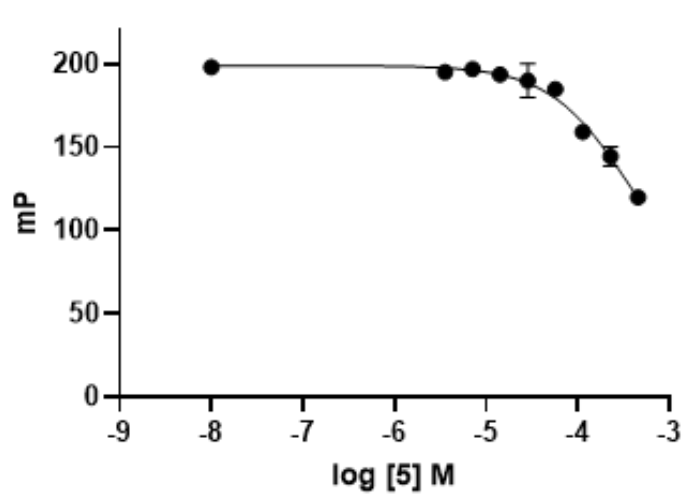

Supplementary Figure S25: Competitive fluorescence polarization data of compound $\mathbf{5}$ with CBX proteins. CBX6 preformed in triplicate, CBX7 and CBX8 preformed in duplicate. A) CBX6 $\left(\log \mathrm{IC}_{50}=-4.756 \mathrm{M}, 95 \% \mathrm{CI}-4.834 \mathrm{M}\right.$ to $\left.\left.-4.678 \mathrm{M}, \mathrm{R}^{2}=0.990\right), \mathrm{B}\right) \mathrm{CBX} 7\left(\log \mathrm{CC}_{50}=-\right.$ $3.672 \mathrm{M}, 95 \% \mathrm{CI}-3.750 \mathrm{M}$ to $\left.\left.-3.590 \mathrm{M}, \mathrm{R}^{2}=0.968\right), \mathrm{C}\right) \mathrm{CBX} 8\left(\log \mathrm{IC}_{50}=-4.778,95 \% \mathrm{CI}-\right.$ $4.949 \mathrm{M}$ to $\left.-4.602 \mathrm{M}, \mathrm{R}^{2}=0.969\right)$. Error bars reported as asymmetrical $95 \%$ confidence intervals. $\mathrm{CBX} 7$ was fitted constraining the $\mathrm{mP}$ value of the endpoint to 100 millipolarization units. 


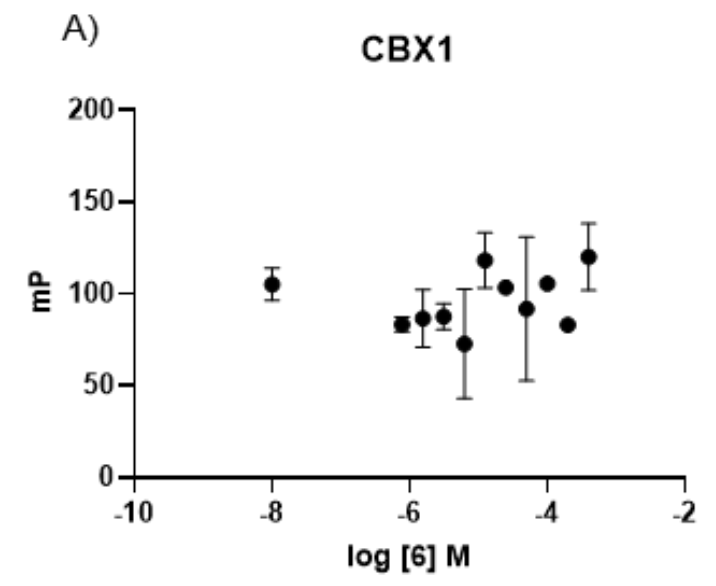

B) $\quad$ CBX6

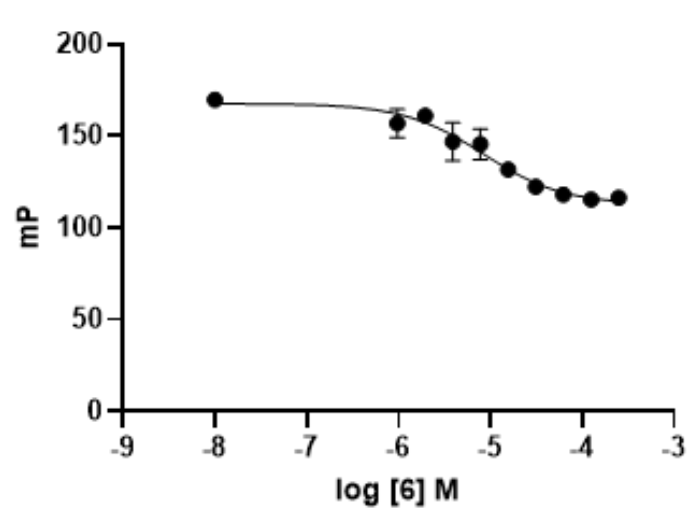

C)

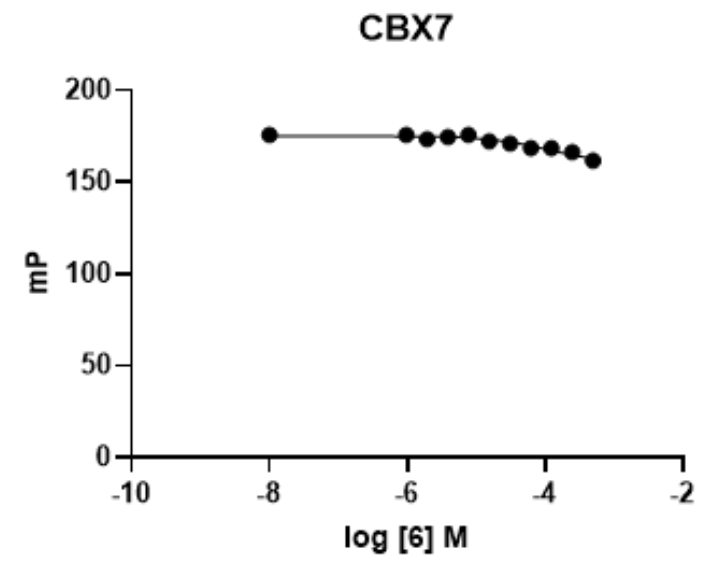

D)

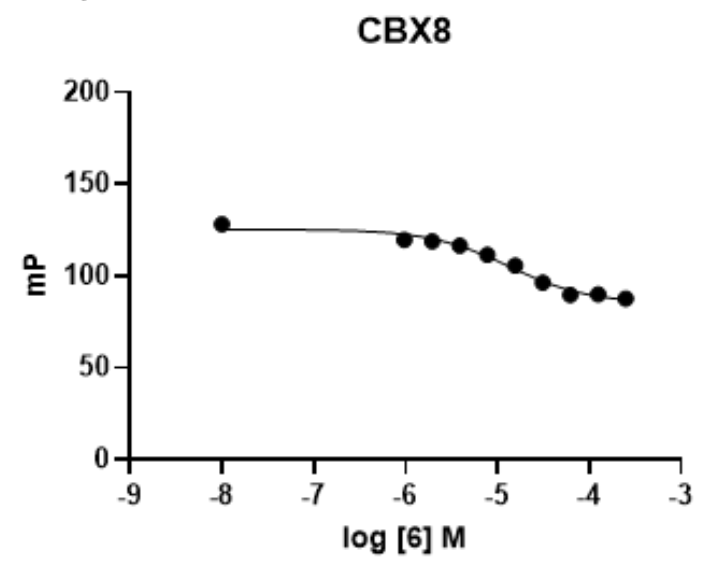

Supplementary Figure S26: Competitive fluorescence polarization data of compound 6 with $\mathrm{CBX}$ proteins. $\mathrm{CBX} 1$ preformed in duplicate; $\mathrm{CBX} 6, \mathrm{CBX} 7$ and $\mathrm{CBX} 8$ performed in triplicate. A) $\mathrm{CBX} 1\left(\log \mathrm{IC}_{50}=>-3.301 \mathrm{M}\right)$, B) $\mathrm{CBX} 6\left(\log \mathrm{IC}_{50}=-5.072 \mathrm{M}, 95 \% \mathrm{CI}-5.276 \mathrm{M}\right.$ to -4.861 $\left.\left.\left.\mathrm{M}, \mathrm{R}^{2}=0.931\right), \mathrm{C}\right) \mathrm{CBX} 7\left(\operatorname{logIC} \mathrm{C}_{50}=>-3.301 \mathrm{M}\right), \mathrm{D}\right) \mathrm{CBX} 8\left(\operatorname{logIC} \mathrm{C}_{50}=-4.871 \mathrm{M}, 95 \% \mathrm{CI}-\right.$ $4.997 \mathrm{M}$ to $\left.-4.745 \mathrm{M}, \mathrm{R}^{2}=0.973\right)$. Error bars reported as asymmetrical $95 \%$ confidence intervals. 
A)

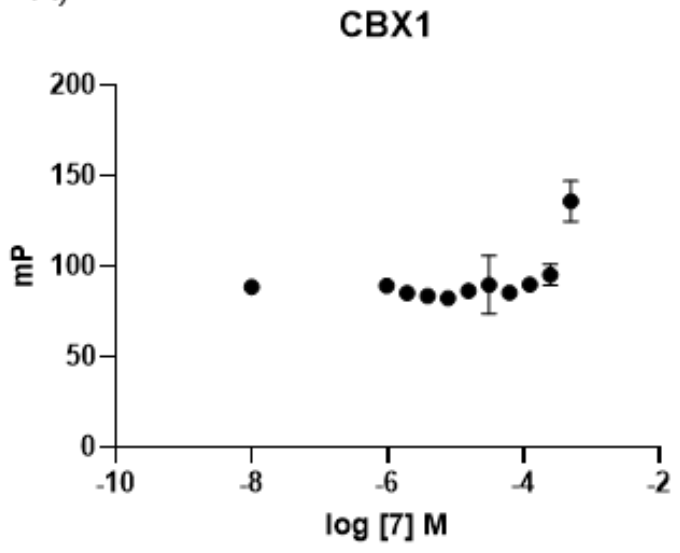

C)

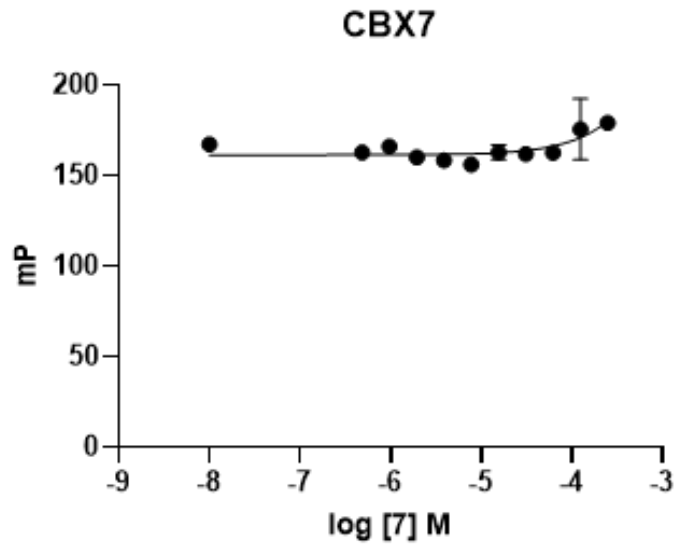

B)

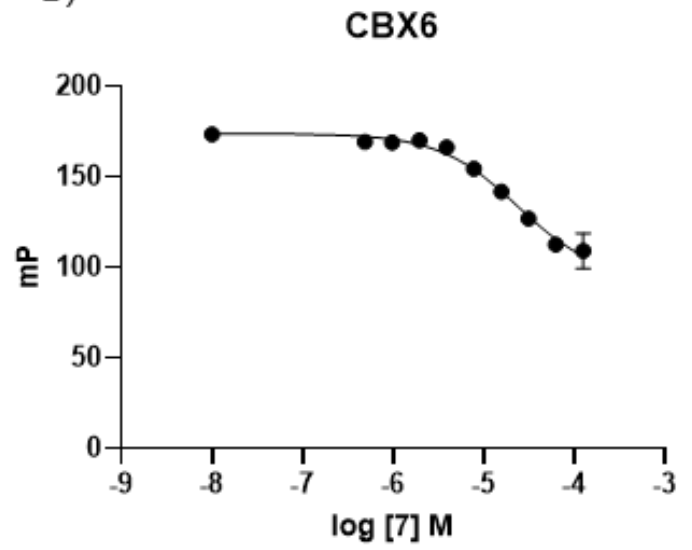

D)

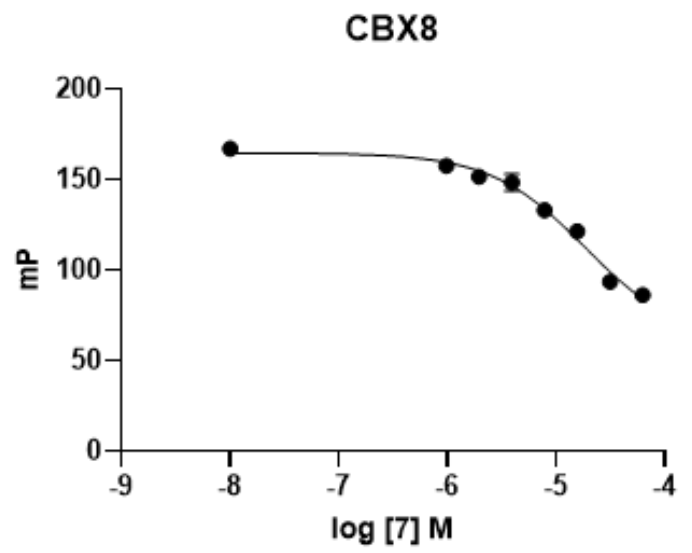

Supplementary Figure S27: Competitive fluorescence polarization data of compound 7 with $\mathrm{CBX}$ proteins performed in triplicate. A) $\left.\mathrm{CBX} 1\left(\log \mathrm{IC}_{50}=>-3.301 \mathrm{M}\right), \mathrm{B}\right) \mathrm{CBX} 6\left(\log \mathrm{IC}_{50}=-\right.$ $4.625 \mathrm{M}, 95 \% \mathrm{CI}-4.754 \mathrm{M}$ to $\left.-4.490 \mathrm{M}, \mathrm{R}^{2}=0.972\right)$, C) $\mathrm{CBX} 7\left(\log \mathrm{IC}_{50}=>-3.301 \mathrm{M}\right)$, D) $\mathrm{CBX} 8\left(\log \mathrm{IC}_{50}=-4.734 \mathrm{M}, 95 \% \mathrm{CI}-4.856 \mathrm{M}\right.$ to $\left.-4.4605 \mathrm{M}, \mathrm{R}^{2}=0.984\right)$. Error bars reported as asymmetrical $95 \%$ confidence intervals. 
A)

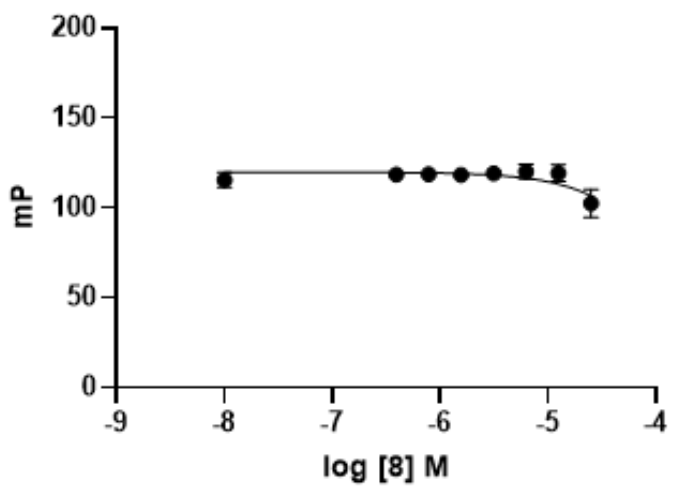

C)

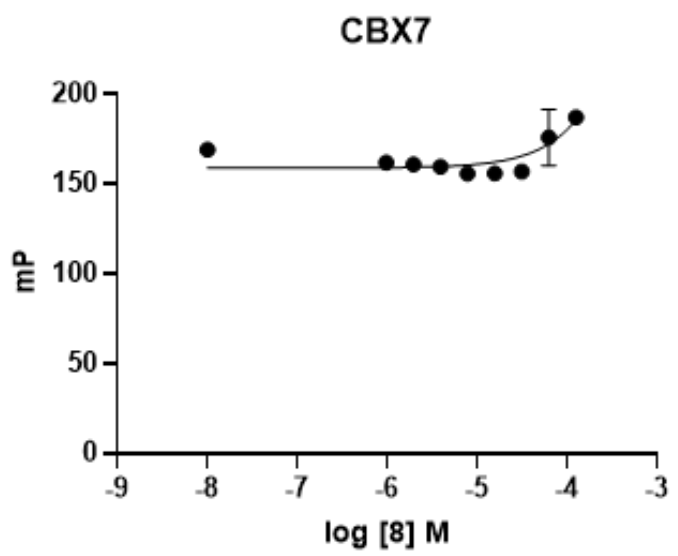

B)

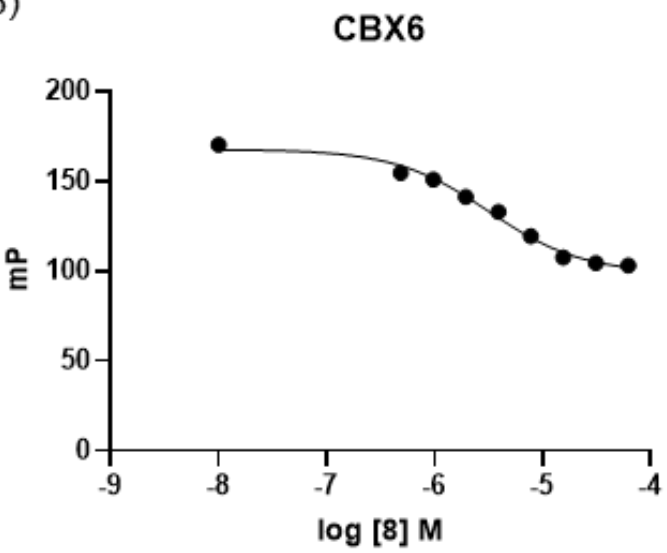

D)

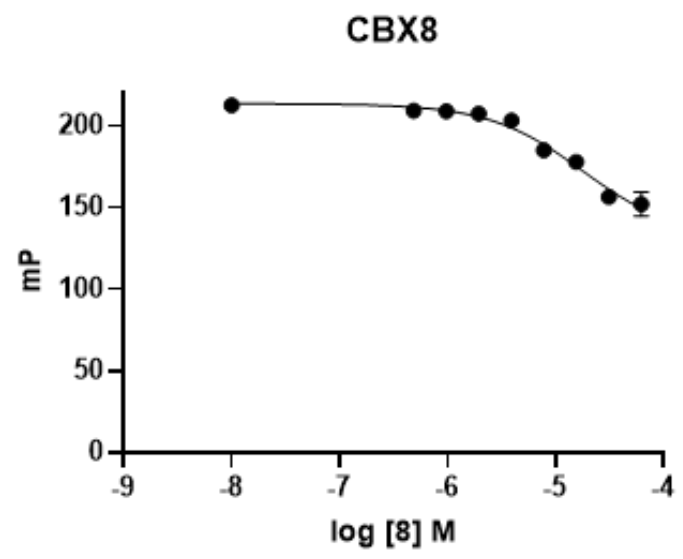

Supplementary Figure S28: Competitive fluorescence polarization data of compound $\mathbf{8}$ with CBX proteins performed in triplicate. A) CBX1 $\left.\left(\log \mathrm{CC}_{50}=>-3.69 \mathrm{M}\right) \mathrm{B}\right) \mathrm{CBX} 6\left(\log \mathrm{C}_{50}=-\right.$ $5.495 \mathrm{M}, 95 \% \mathrm{CI}-5.591 \mathrm{M}$ to $\left.\left.\left.-5.398 \mathrm{M}, \mathrm{R}^{2}=0.985\right), \mathrm{C}\right) \mathrm{CBX} 7\left(\log \mathrm{IC}_{50}=>-3.69 \mathrm{M}\right), \mathrm{D}\right)$ CBX8 $\left(\log \mathrm{IC}_{50}=-4.746 \mathrm{M}, 95 \% \mathrm{CI}-4.896 \mathrm{M}\right.$ to $\left.-4.586 \mathrm{M}, \mathrm{R}^{2}=0.969\right)$. Error bars reported as asymmetrical $95 \%$ confidence intervals. Each assay was performed with 5\% DMSO due to solubility issues of the inhibitor. 
A)

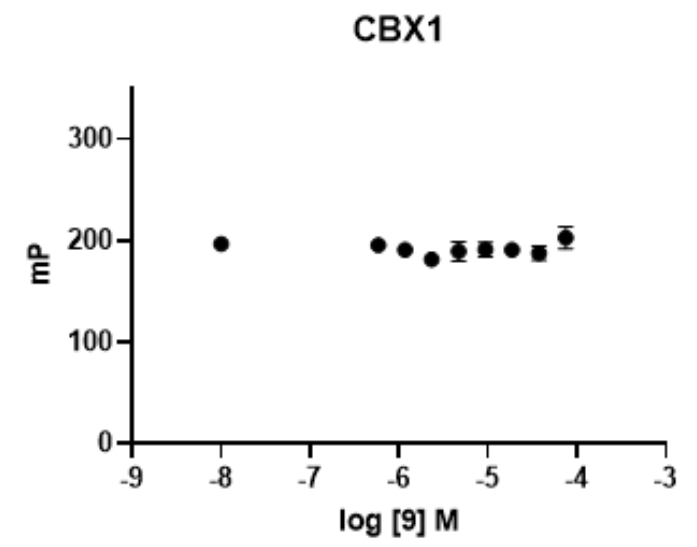

C)

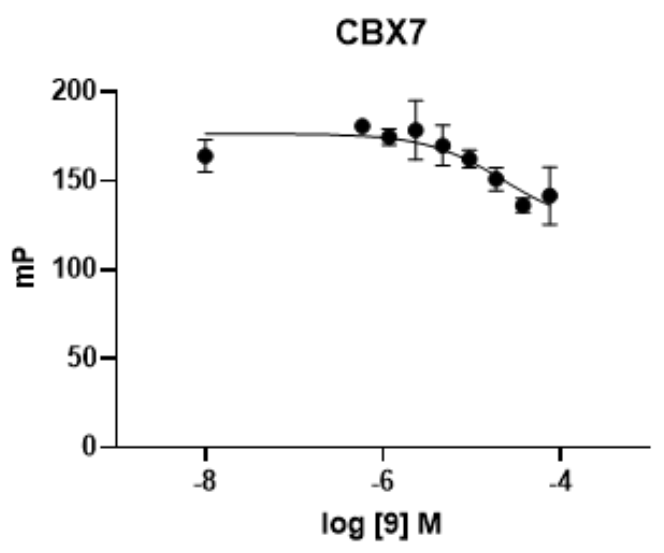

B)

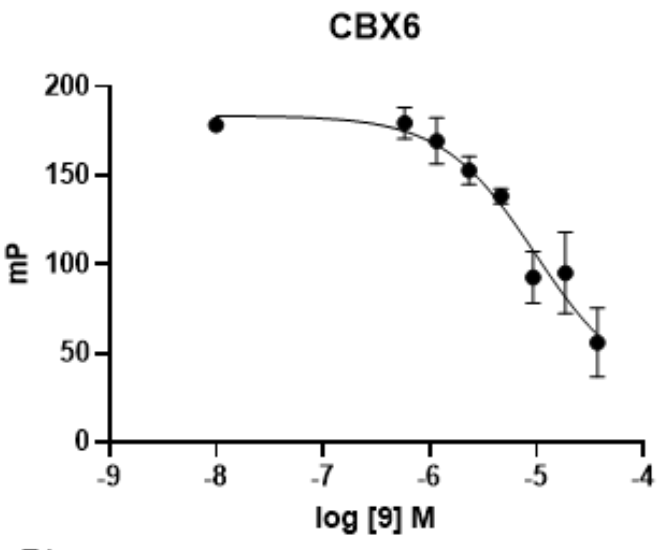

D)

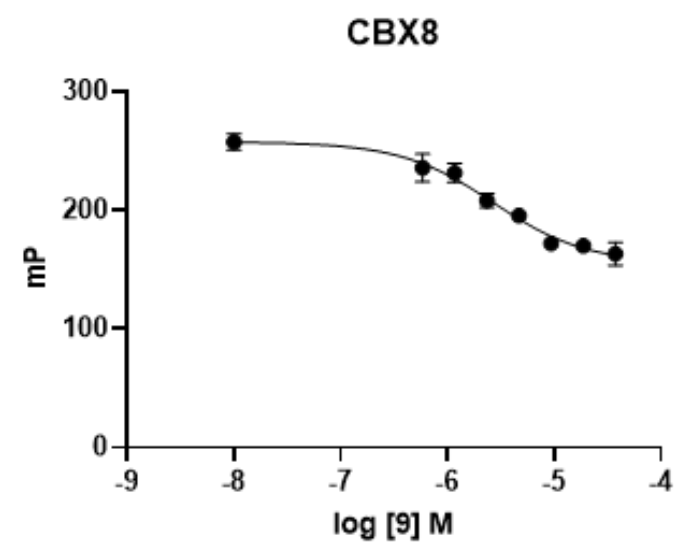

Supplementary Figure S29: Competitive fluorescence polarization data of compound 9 with $\mathrm{CBX}$ proteins. $\mathrm{CBX} 1$ and $\mathrm{CBX} 8$ preformed in triplicate, $\mathrm{CBX} 6$ and $\mathrm{CBX} 7$ preformed in triplicate. A) CBX1 $\left.\left(\operatorname{logIC} \mathrm{C}_{50}=>-3.522 \mathrm{M}\right) \mathrm{B}\right) \mathrm{CBX} 6\left(\log \mathrm{IC}_{50}=-5.178 \mathrm{M}, 95 \% \mathrm{CI}-5.235 \mathrm{M}\right.$ to $\left.\left.\left.-5.120 \mathrm{M}, \mathrm{R}^{2}=0.995\right), \mathrm{C}\right) \mathrm{CBX} 7\left(\log \mathrm{IC}_{50}=>-3.602 \mathrm{M}\right), \mathrm{D}\right) \mathrm{CBX} 8\left(\log \mathrm{IC}_{50}=-5.342 \mathrm{M}, 95 \%\right.$ $\mathrm{CI}-5.525 \mathrm{M}$ to $\left.-5.147 \mathrm{M}, \mathrm{R}^{2}=0.947\right)$. Error bars reported as asymmetrical $95 \%$ confidence intervals. 
A)

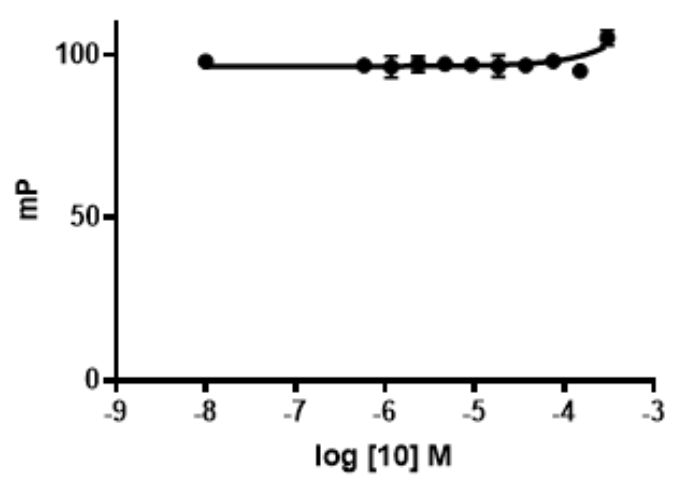

C)

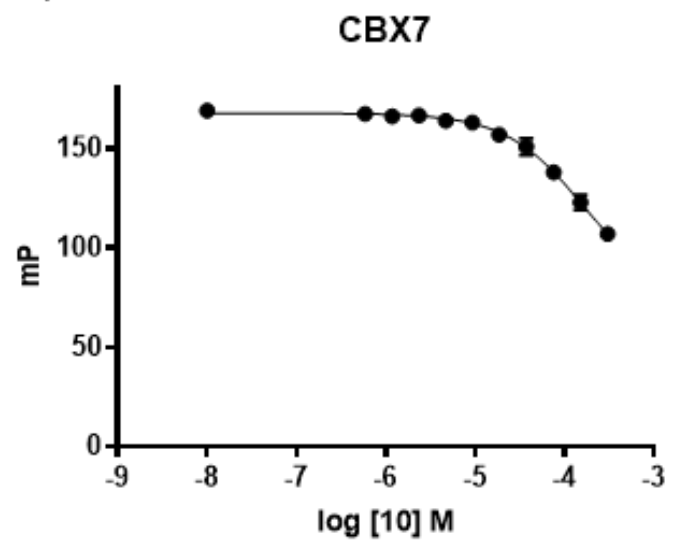

B)

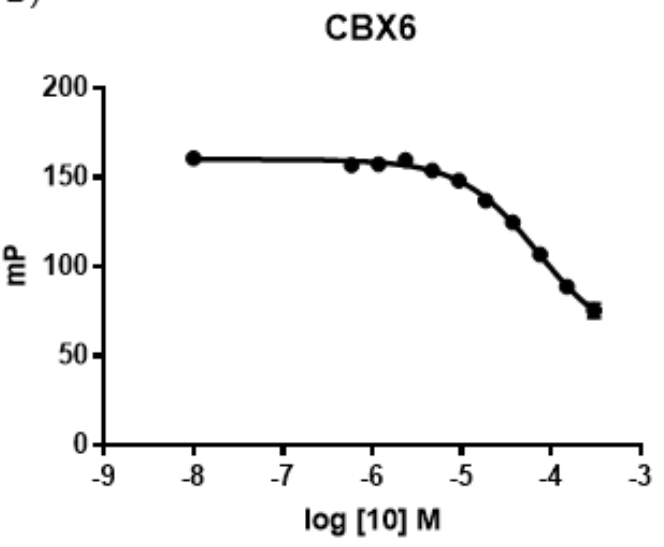

D)

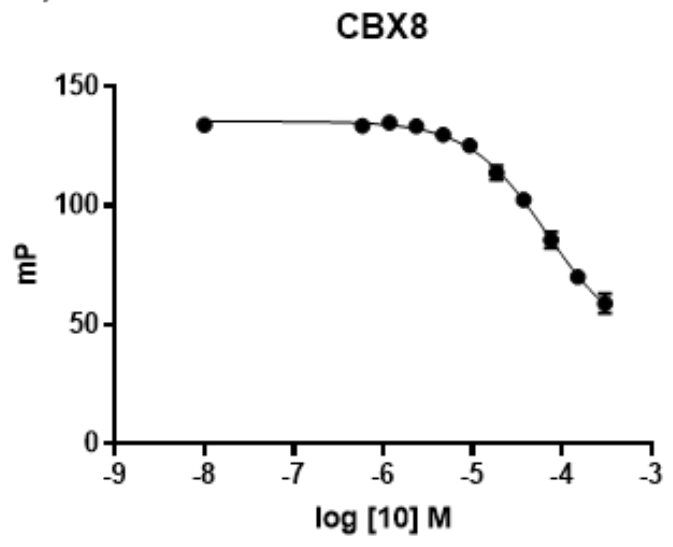

Supplementary Figure S30: Competitive fluorescence polarization data of compound 10 with CBX proteins performed in triplicate. A) CBX1 $\left.\left(\operatorname{logIC} \mathrm{C}_{50}=>-3.522 \mathrm{M}\right) \mathrm{B}\right) \mathrm{CBX} 6\left(\log \mathrm{C}_{50}=-\right.$ $4.143 \mathrm{M}, 95 \% \mathrm{CI}-4.205 \mathrm{M}$ to $\left.\left.-4080 \mathrm{M}, \mathrm{R}^{2}=0.994\right), \mathrm{C}\right) \mathrm{CBX} 7\left(\log \mathrm{IC}_{50}=-3.791 \mathrm{M}, 95 \% \mathrm{CI}-\right.$ $3.892 \mathrm{M}$ to $\left.-3.683 \mathrm{M}, \mathrm{R}^{2}=0.989\right)$, D) $\mathrm{CBX} 8\left(\log \mathrm{IC}_{50}=-4.169 \mathrm{M}, 95 \% \mathrm{CI}-4.238 \mathrm{M}\right.$ to -4.099 $\left.\mathrm{M}, \mathrm{R}^{2}=0.993\right)$. Error bars reported as asymmetrical $95 \%$ confidence intervals. 
A)

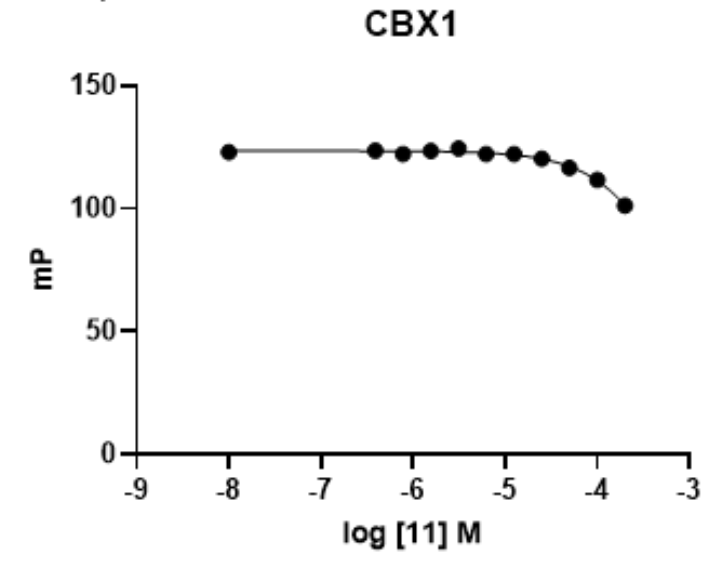

C)

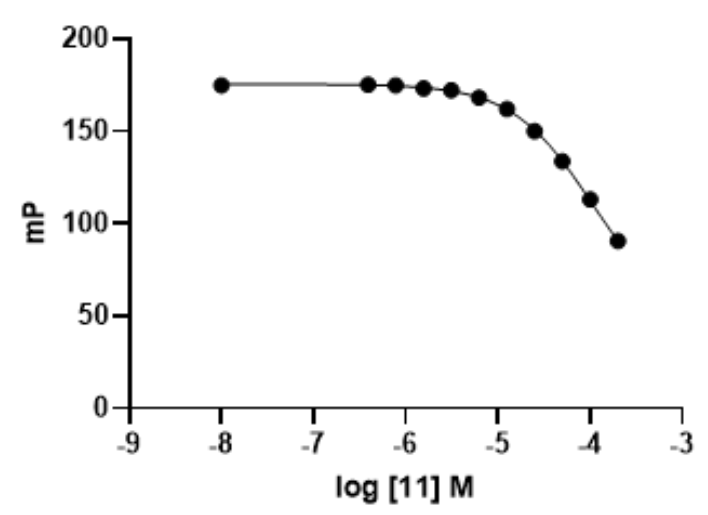

B)

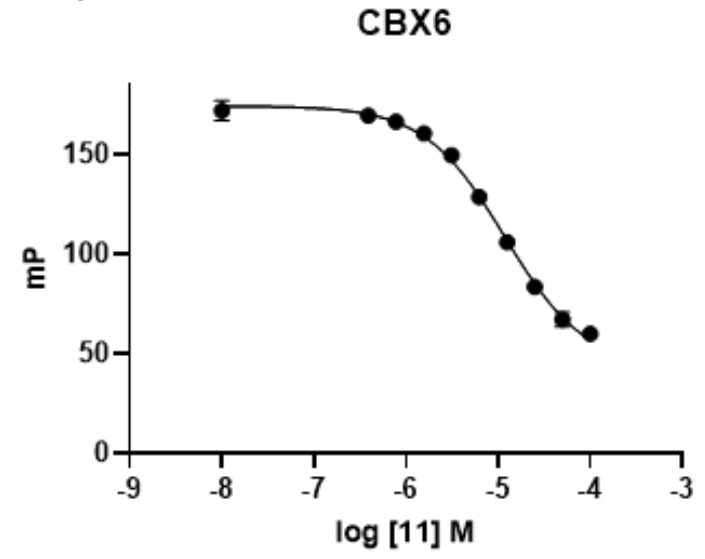

D)

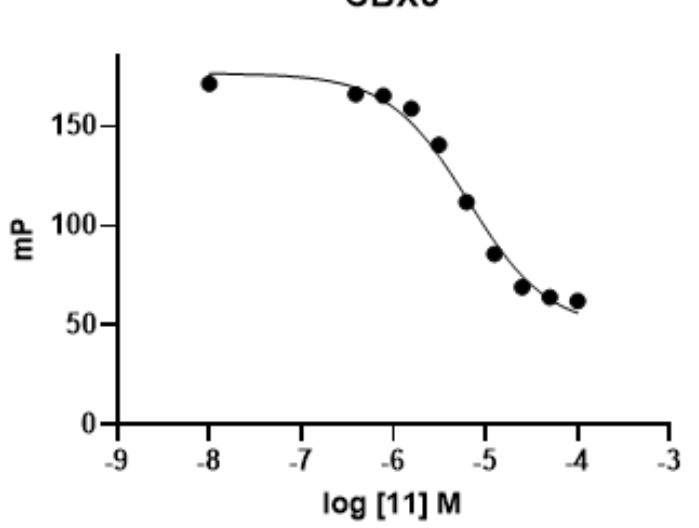

Supplementary Figure S31: Competitive fluorescence polarization data of compound 11 with CBX proteins performed in triplicate. A) CBX1 $\left.\left(\log \mathrm{IC}_{50}=>-3.522 \mathrm{M}\right) \mathrm{B}\right) \mathrm{CBX} 6\left(\log \mathrm{CC}_{50}=-\right.$ $4.982 \mathrm{M}, 95 \% \mathrm{CI}-4.983 \mathrm{M}$ to $\left.\left.-4.872 \mathrm{M}, \mathrm{R}^{2}=0.995\right), \mathrm{C}\right) \mathrm{CBX} 7\left(\log \mathrm{IC}_{50}=-3.978 \mathrm{M}, 95 \% \mathrm{CI}-\right.$ $4.013 \mathrm{M}$ to $\left.\left.-3.941 \mathrm{M}, \mathrm{R}^{2}=0.998\right), \mathrm{D}\right) \mathrm{CBX} 8\left(\log \mathrm{IC}_{50}=-5.186 \mathrm{M}, 95 \% \mathrm{CI}-5.265 \mathrm{M}\right.$ to -5.106 $\left.\mathrm{M}, \mathrm{R}^{2}=0.987\right)$. Error bars reported as asymmetrical $95 \%$ confidence intervals. 
A)

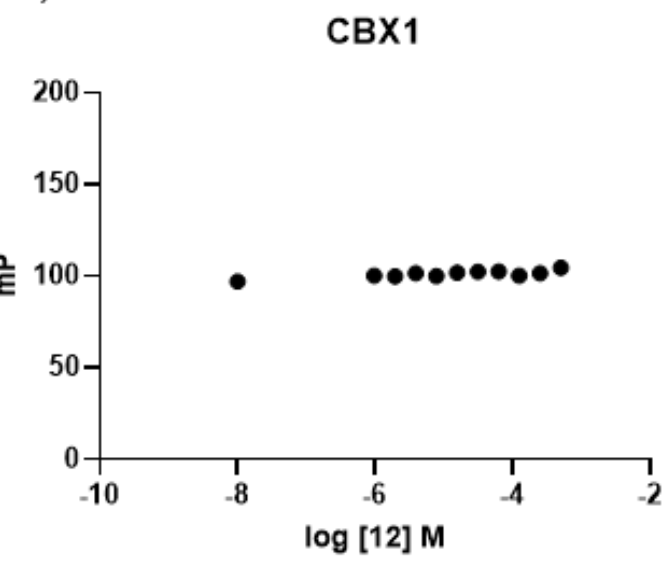

C)

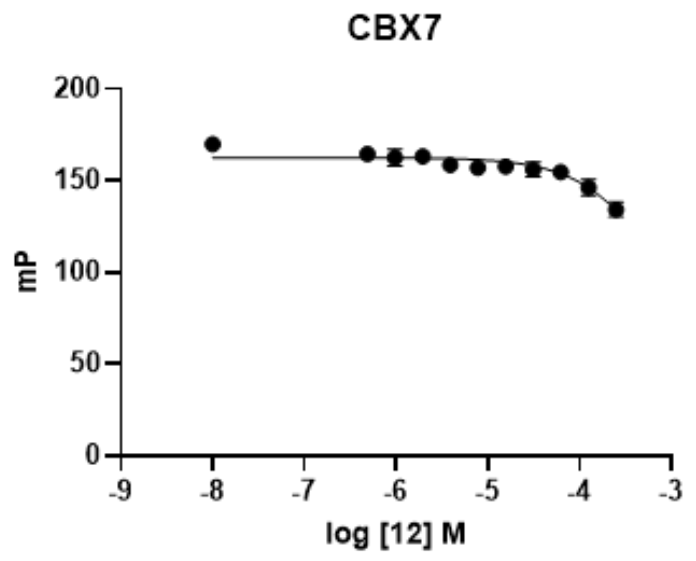

B)

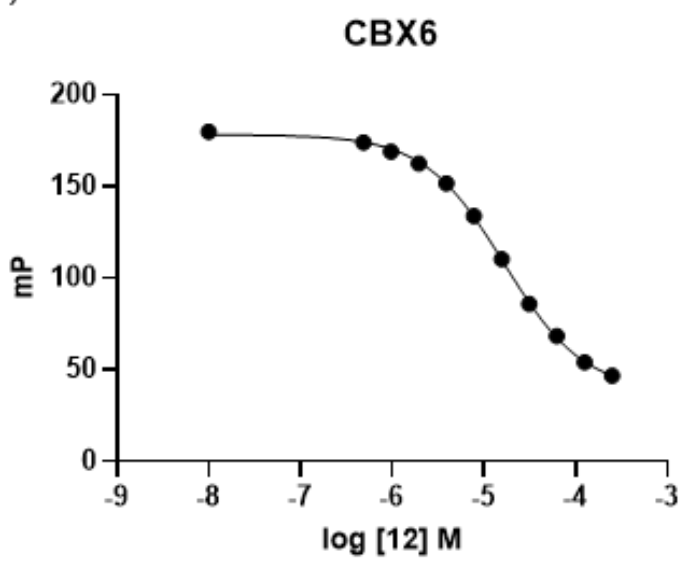

D)

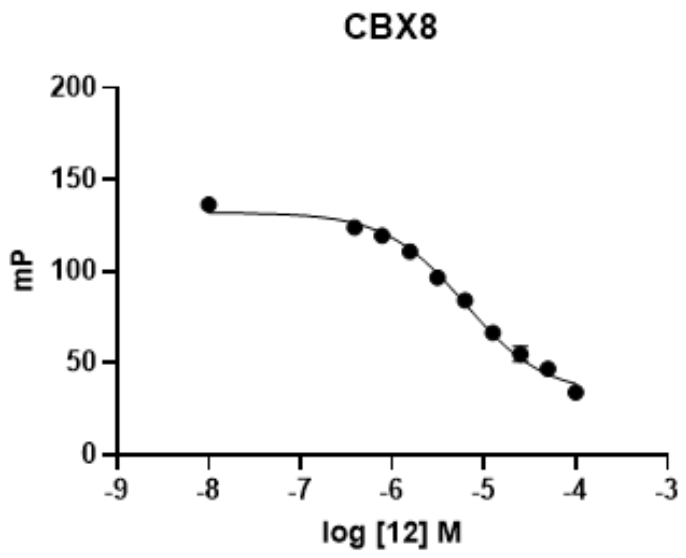

Supplementary Figure S32: Competitive fluorescence polarization data of compound 12 with CBX proteins performed in triplicate. A) $\left.\mathrm{CBX} 1\left(\log \mathrm{IC}_{50}=>-3.301 \mathrm{M}\right) \mathrm{B}\right) \mathrm{CBX} 6\left(\log \mathrm{CC}_{50}=-\right.$ 4.781 M, 95\% CI -4.804 M to $\left.-4.758 \mathrm{M}, \mathrm{R}^{2}=0.999\right)$, C) $\mathrm{CBX} 7\left(\log \mathrm{IC}_{50}=>-3.522 \mathrm{M}\right)$, D) $\mathrm{CBX} 8\left(\log \mathrm{IC}_{50}=-5.207 \mathrm{M}, 95 \% \mathrm{CI}-5.284 \mathrm{M}\right.$ to $\left.-5.130 \mathrm{M}, \mathrm{R}^{2}=0.991\right)$. Error bars reported as asymmetrical $95 \%$ confidence intervals. 
A)

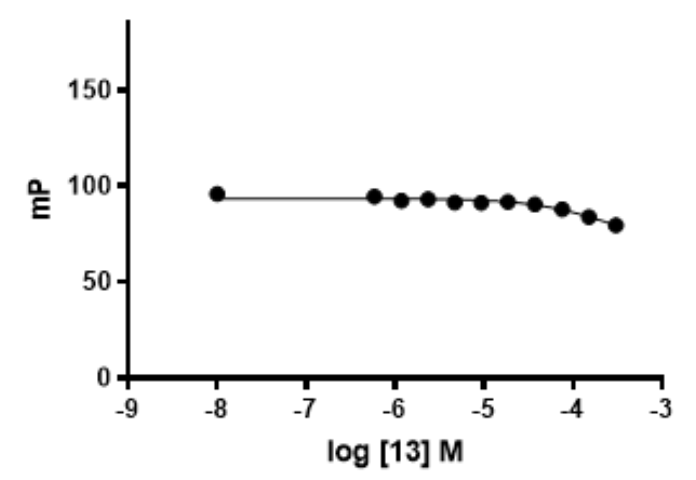

C)

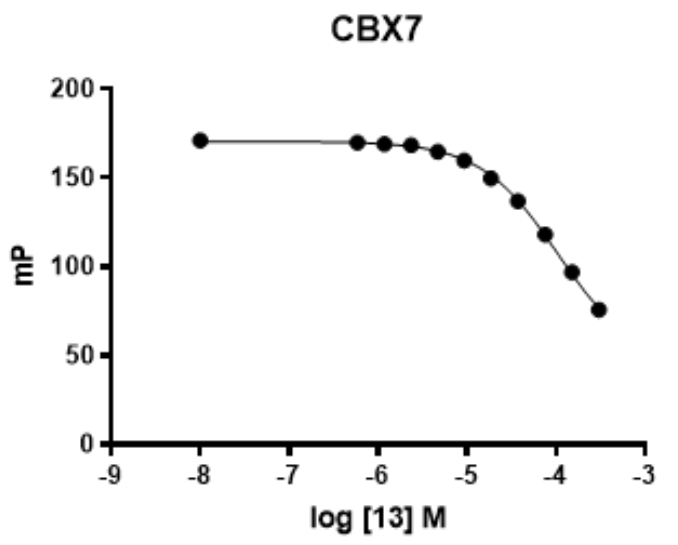

B)

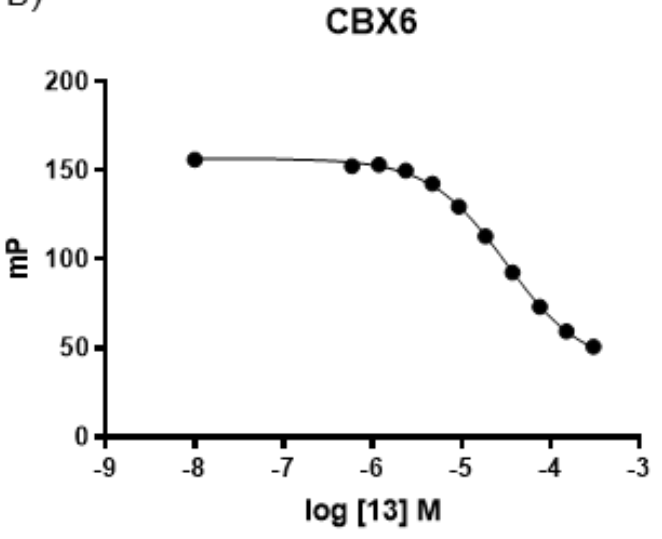

D)

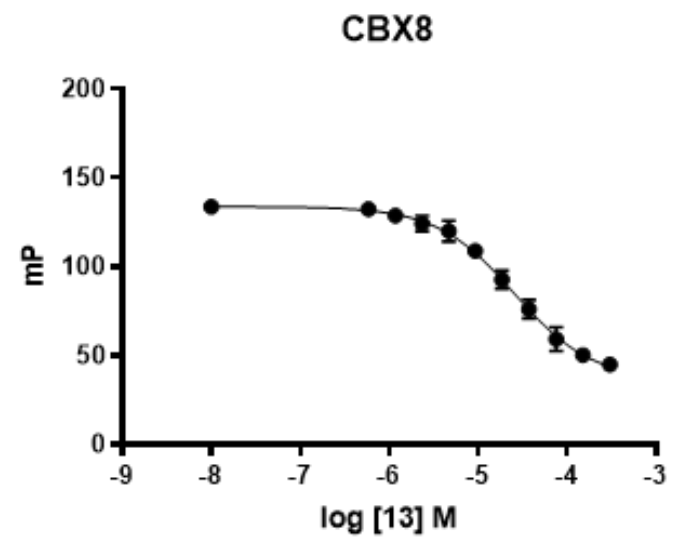

Supplementary Figure S33: Competitive fluorescence polarization data of compound 13 with $\mathrm{CBX}$ proteins performed in triplicate. A) $\left.\mathrm{CBX} 1\left(\operatorname{logIC} \mathrm{C}_{50}=>-3.522 \mathrm{M}\right) \mathrm{B}\right) \mathrm{CBX} 6\left(\operatorname{logIC} \mathrm{C}_{50}=-\right.$ $4.502 \mathrm{M}, 95 \% \mathrm{CI}-4.528 \mathrm{M}$ to $\left.-4.475 \mathrm{M}, \mathrm{R}^{2}=0.999\right)$, C) $\mathrm{CBX} 7\left(\log \mathrm{IC}_{50}=-3.981 \mathrm{M}, 95 \% \mathrm{CI}-\right.$ 4.030 M to $\left.-3.932 \mathrm{M}, \mathrm{R}^{2}=0.997\right)$, D) $\mathrm{CBX} 8\left(\log \mathrm{IC}_{50}=-4.594 \mathrm{M}, 95 \% \mathrm{CI}-4.673 \mathrm{M}\right.$ to -4.515 $\left.\mathrm{M}, \mathrm{R}^{2}=0.992\right)$. Error bars reported as asymmetrical $95 \%$ confidence intervals. 
A)

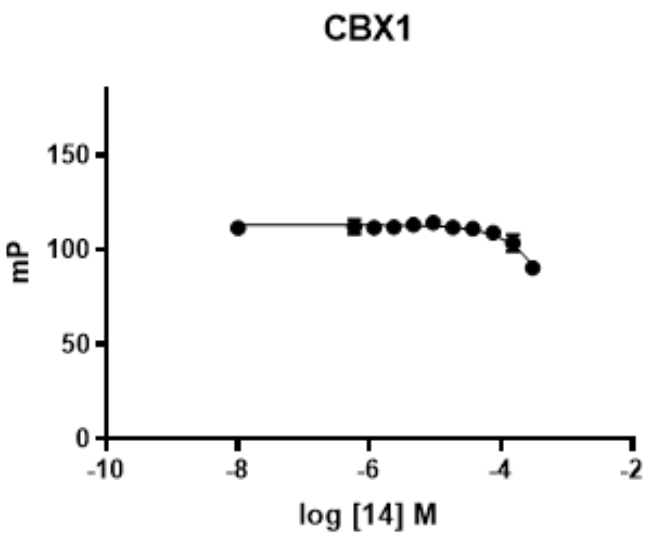

C)

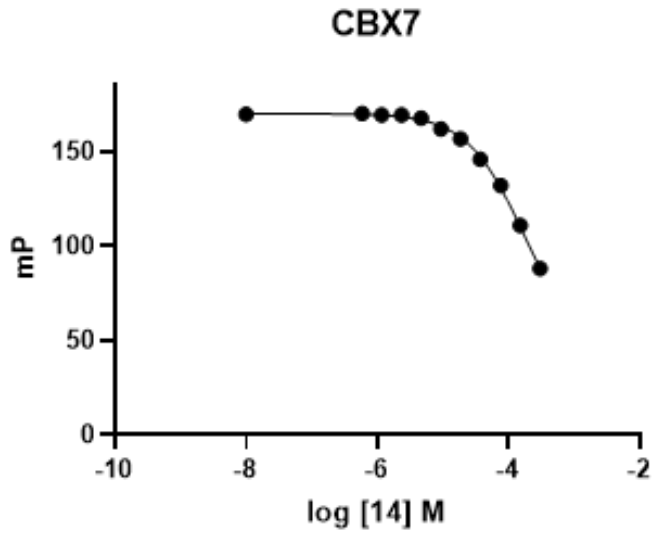

B)

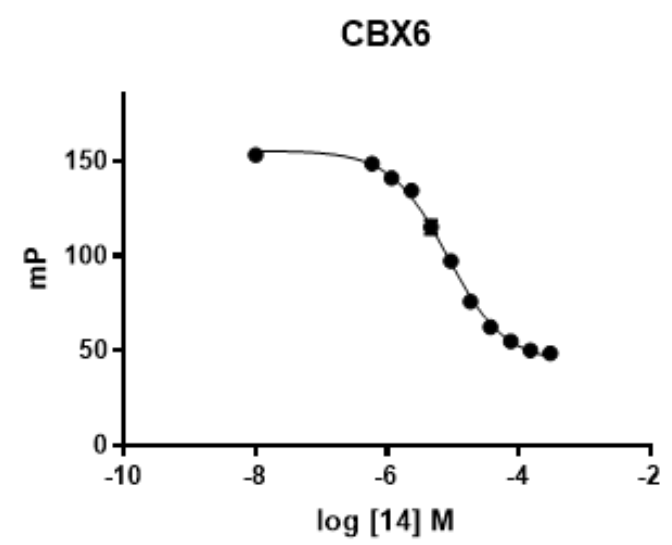

D)

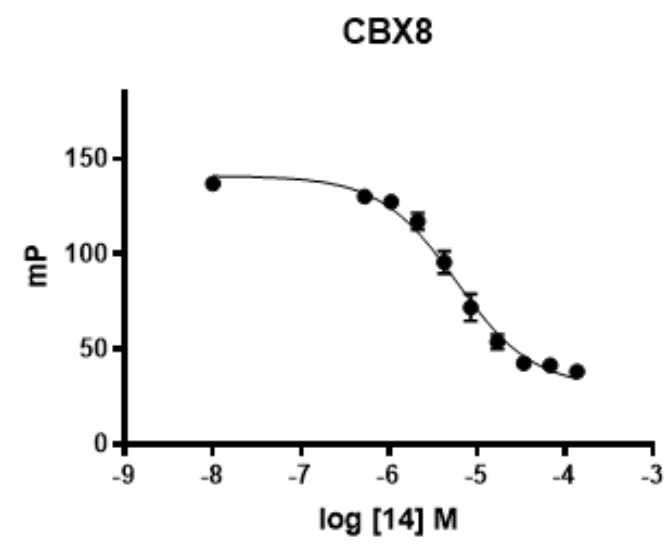

Supplementary Figure S34: Competitive fluorescence polarization data of compound 14 with CBX proteins performed in triplicate. A) CBX1 $\left.\left(\log \mathrm{IC}_{50}=>-3.522 \mathrm{M}\right) \mathrm{B}\right) \mathrm{CBX} 6\left(\log \mathrm{CC}_{50}=-\right.$ $5.080 \mathrm{M}, 95 \% \mathrm{CI}-5.120 \mathrm{M}$ to $\left.\left.-5.039 \mathrm{M}, \mathrm{R}^{2}=0.997\right), \mathrm{C}\right) \mathrm{CBX} 7\left(\log \mathrm{IC}_{50}=-3.676 \mathrm{M}, 95 \% \mathrm{CI}-\right.$ $3.833 \mathrm{M}$ to $\left.-3.698 \mathrm{M}, \mathrm{R}^{2}=0.996\right)$, D) $\mathrm{CBX} 8\left(\log \mathrm{IC}_{50}=-5.234 \mathrm{M}, 95 \% \mathrm{CI}-5.317 \mathrm{M}\right.$ to -5.150 $\left.\mathrm{M}, \mathrm{R}^{2}=0.985\right)$. Error bars reported as asymmetrical $95 \%$ confidence intervals. 
A)

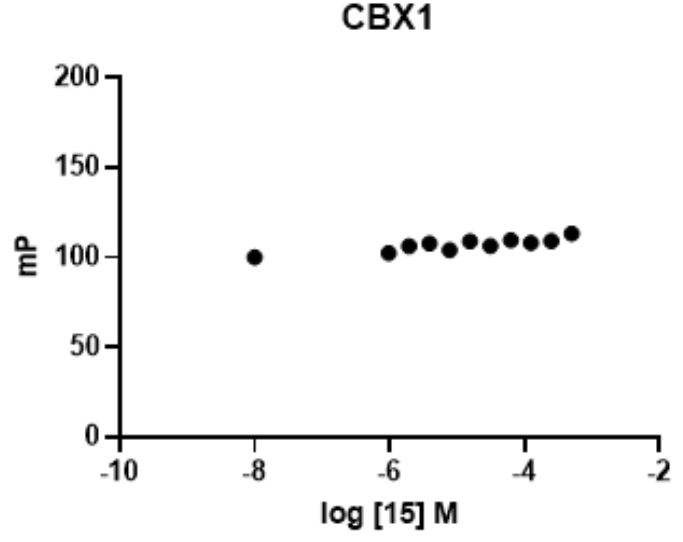

C)

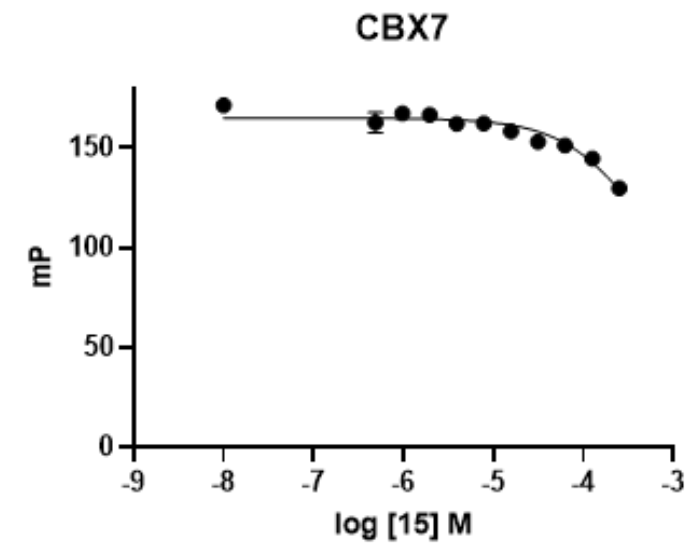

B)

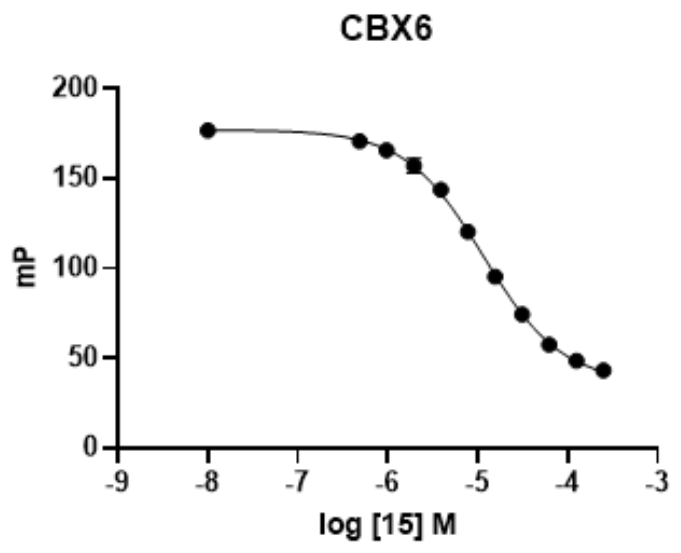

D)

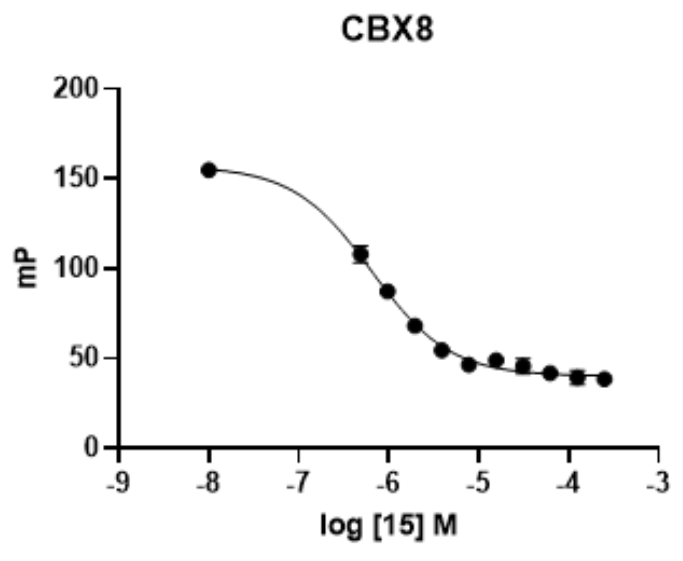

Supplementary Figure S35: Competitive fluorescence polarization data of compound 15 with CBX proteins performed in triplicate. A) $\left.\mathrm{CBX} 1\left(\log \mathrm{CC}_{50}=>-3.301 \mathrm{M}\right) \mathrm{B}\right) \mathrm{CBX} 6\left(\log \mathrm{CC}_{50}=-\right.$ $4.934 \mathrm{M}, 95 \% \mathrm{CI}-4.969 \mathrm{M}$ to $\left.-4.899 \mathrm{M}, \mathrm{R}^{2}=0.998\right)$, C) $\mathrm{CBX} 7\left(\log \mathrm{IC}_{50}=>-3.602 \mathrm{M}\right)$, D) CBX8 $\left(\log \mathrm{IC}_{50}=-6.189 \mathrm{M}, 95 \% \mathrm{CI}-6.239 \mathrm{M}\right.$ to $\left.-6.138 \mathrm{M}, \mathrm{R}^{2}=0.992\right)$. Error bars reported as asymmetrical $95 \%$ confidence intervals. 
A)

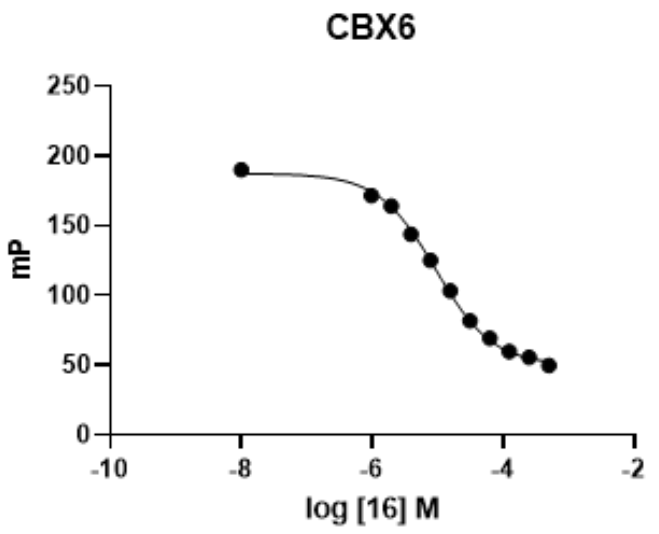

C)

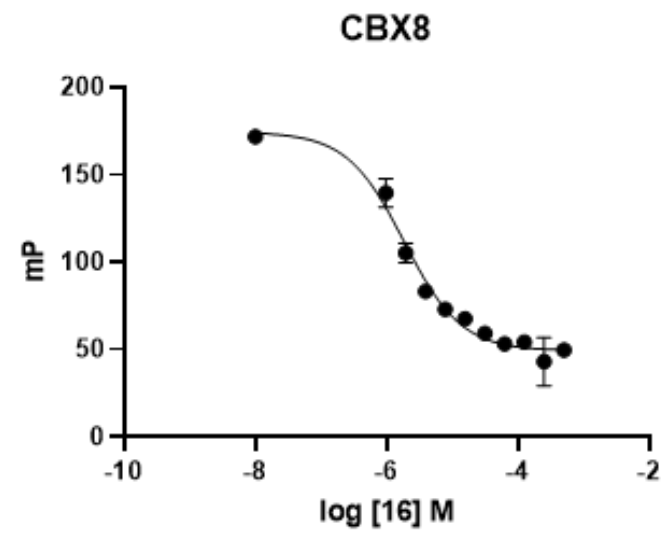

B)

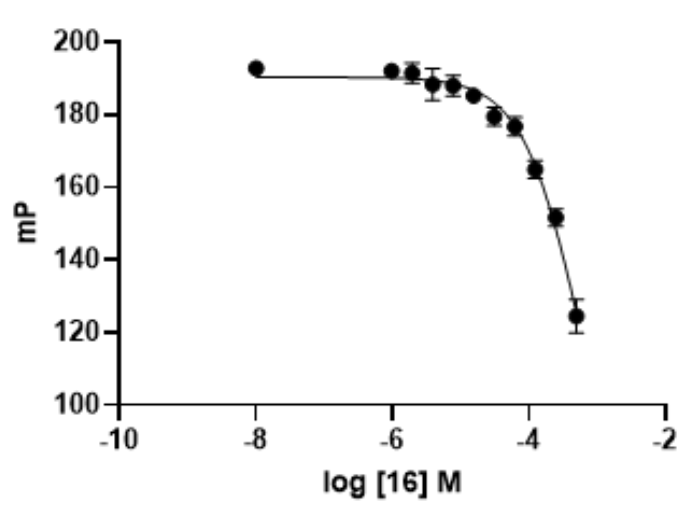

Supplementary Figure S36: Competitive fluorescence polarization data of compound $\mathbf{1 6}$ with CBX proteins performed in triplicate. A) CBX6 $\left(\operatorname{logIC}{ }_{50}=-5.030 \mathrm{M}, 95 \% \mathrm{CI}-5.073 \mathrm{M}\right.$ to $\left.\left.4.986 \mathrm{M}, \mathrm{R}^{2}=0.996\right), \mathrm{B}\right) \mathrm{CBX} 7\left(\operatorname{logIC} 50=-3.216 \mathrm{M}, 95 \% \mathrm{CI}-3.414 \mathrm{M}\right.$ to $-2.955 \mathrm{M}, \mathrm{R}^{2}=$ $0.977), \mathrm{C}) \mathrm{CBX} 8\left(\operatorname{logIC} \mathrm{I}_{50}=-5.734,95 \% \mathrm{CI}-5.824 \mathrm{M}\right.$ to $\left.-5.641 \mathrm{M}, \mathrm{R}^{2}=0.974\right)$. Error bars reported as asymmetrical $95 \%$ confidence intervals. 
A)

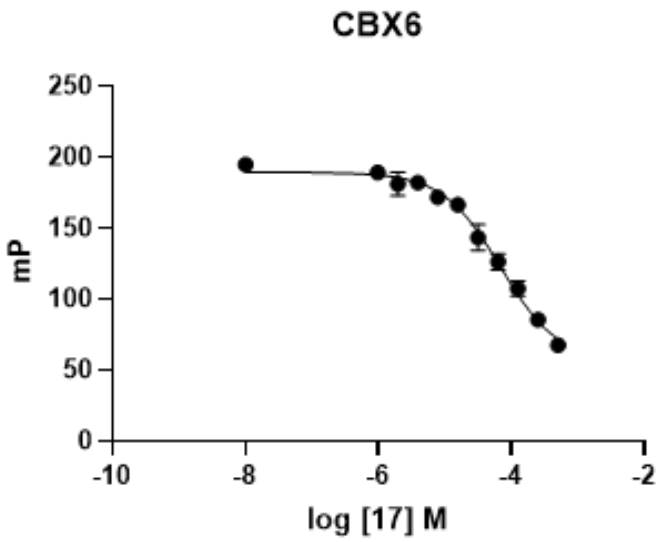

C)

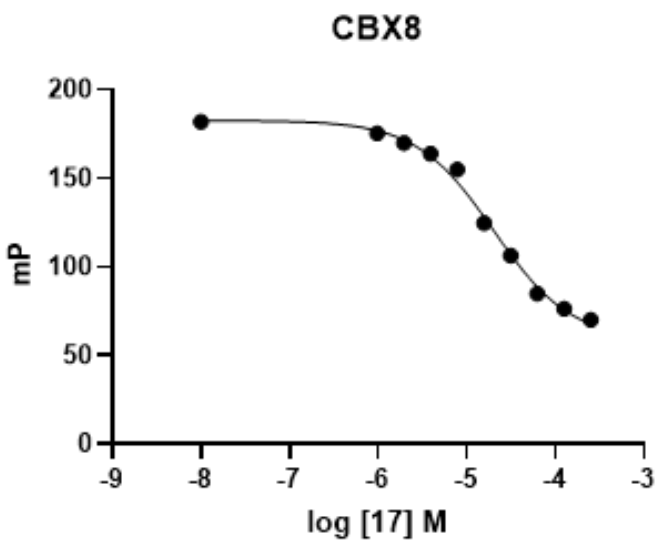

B)

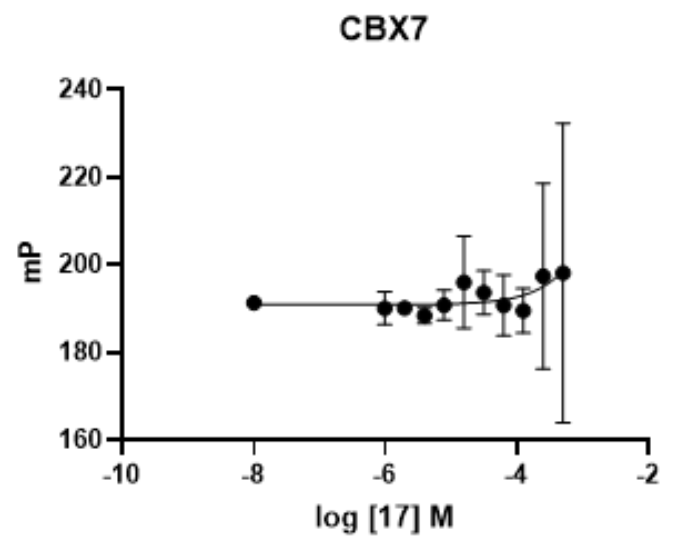

Supplementary Figure S37: Competitive fluorescence polarization data of compound 17 with CBX proteins performed in triplicate. A) CBX6 $\left(\log \mathrm{IC}_{50}=-4.165 \mathrm{M}, 95 \% \mathrm{CI}-4.270 \mathrm{M}\right.$ to $\left.\left.\left.4.058 \mathrm{M}, \mathrm{R}^{2}=0.984\right), \mathrm{B}\right) \mathrm{CBX} 7\left(\log \mathrm{IC}_{50}=>-3.301 \mathrm{M}\right), \mathrm{C}\right) \mathrm{CBX} 8\left(\log \mathrm{IC}_{50}=-4.699,95 \% \mathrm{CI}-\right.$ $4.773 \mathrm{M}$ to $\left.-4.624 \mathrm{M}, \mathrm{R}^{2}=0.994\right)$. Error bars reported as asymmetrical $95 \%$ confidence intervals. 
A)

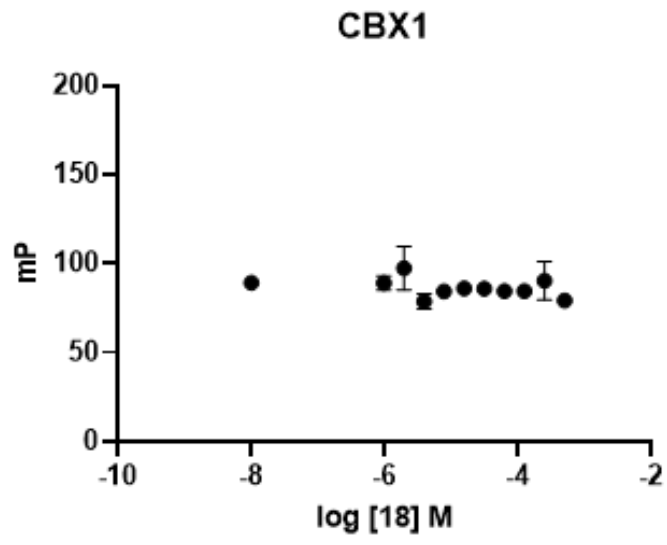

C)

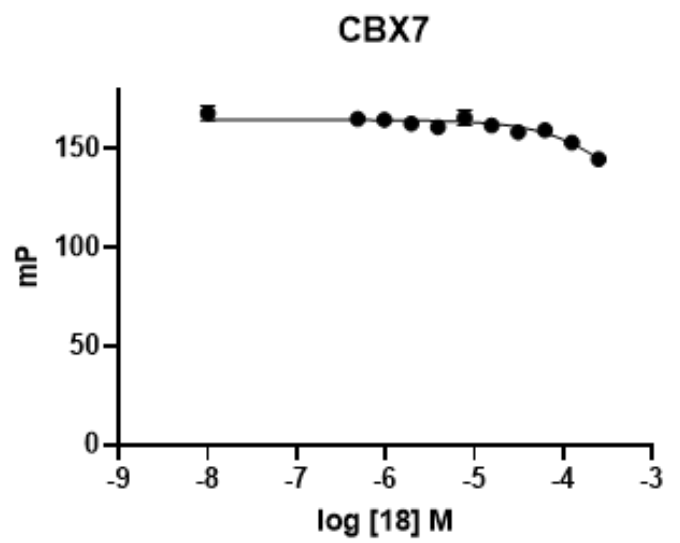

B)

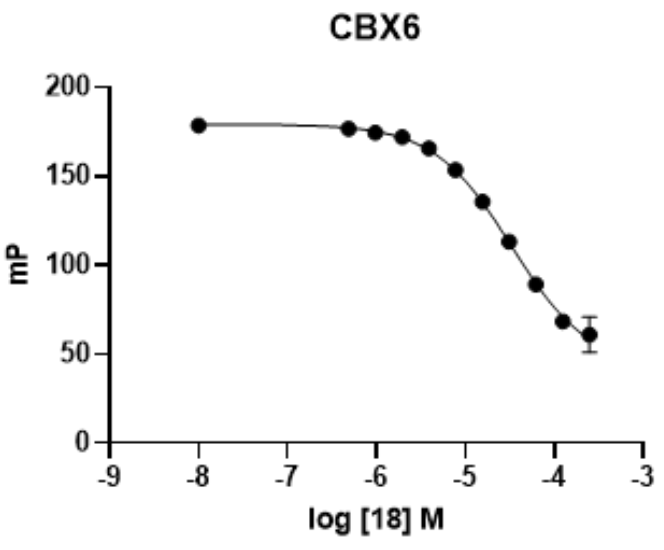

D)

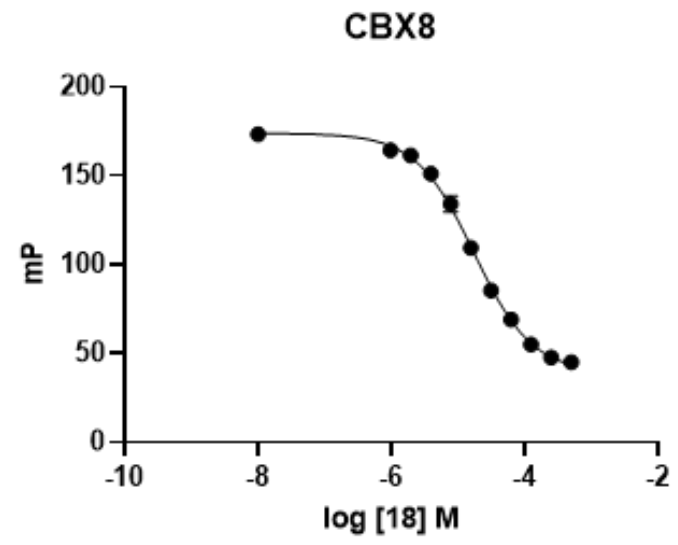

Supplementary Figure S38: Competitive fluorescence polarization data of compound 18 with $\mathrm{CBX}$ proteins performed in triplicate. A) CBX1 $\left.\left(\log \mathrm{CC}_{50}=>-3.301 \mathrm{M}\right) \mathrm{B}\right) \mathrm{CBX} 6\left(\log \mathrm{IC}_{50}=-\right.$ $4.480 \mathrm{M}, 95 \% \mathrm{CI}-4.535 \mathrm{M}$ to $\left.\left.\left.-4.425 \mathrm{M}, \mathrm{R}^{2}=0.995\right), \mathrm{C}\right) \mathrm{CBX} 7\left(\log \mathrm{IC}_{50}=>-3.602 \mathrm{M}\right), \mathrm{D}\right)$ CBX8 $\left(\log \mathrm{IC}_{50}=-4.758 \mathrm{M}, 95 \% \mathrm{CI}-4.800 \mathrm{M}\right.$ to $\left.-4.715 \mathrm{M}, \mathrm{R}^{2}=0.996\right)$. Error bars reported as asymmetrical $95 \%$ confidence intervals. 
A)

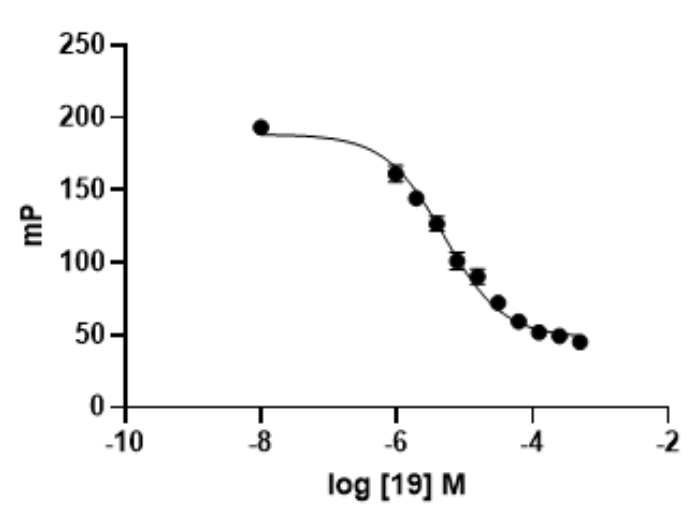

C)

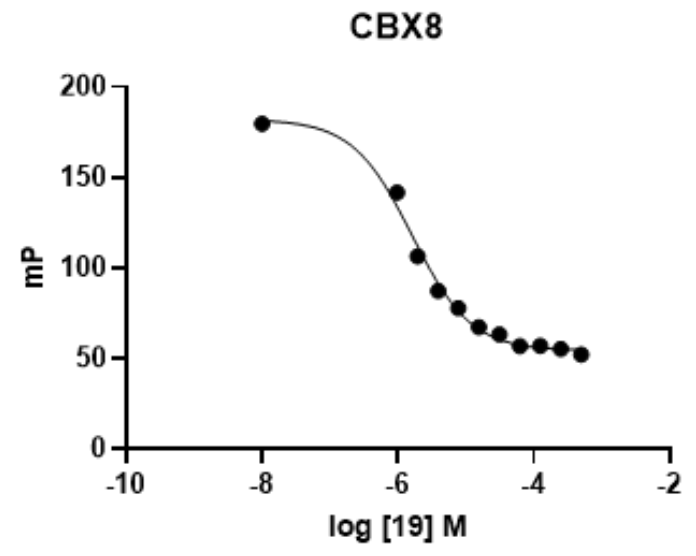

B)

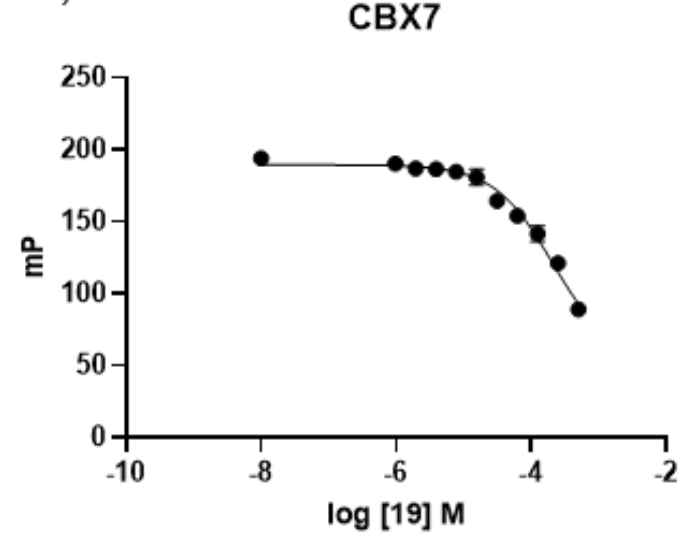

Supplementary Figure S39: Competitive fluorescence polarization data of compound 19 with CBX proteins performed in triplicate. A) CBX6 $\left(\operatorname{logIC} \mathrm{C}_{50}=-5.303 \mathrm{M}, 95 \% \mathrm{CI}-5.379 \mathrm{M}\right.$ to $\left.\left.5.224 \mathrm{M}, \mathrm{R}^{2}=0.988\right), \mathrm{B}\right) \mathrm{CBX} 7\left(\operatorname{logIC} 50=-3.679 \mathrm{M}, 95 \% \mathrm{CI}-3.823 \mathrm{M}\right.$ to $-3.523 \mathrm{M}, \mathrm{R}^{2}=$ $0.979), \mathrm{C}) \mathrm{CBX} 8\left(\log \mathrm{IC}_{50}=-5.792,95 \% \mathrm{CI}-5.846 \mathrm{M}\right.$ to $\left.-5.737 \mathrm{M}, \mathrm{R}^{2}=0.990\right)$. Error bars reported as asymmetrical $95 \%$ confidence intervals. 
A)

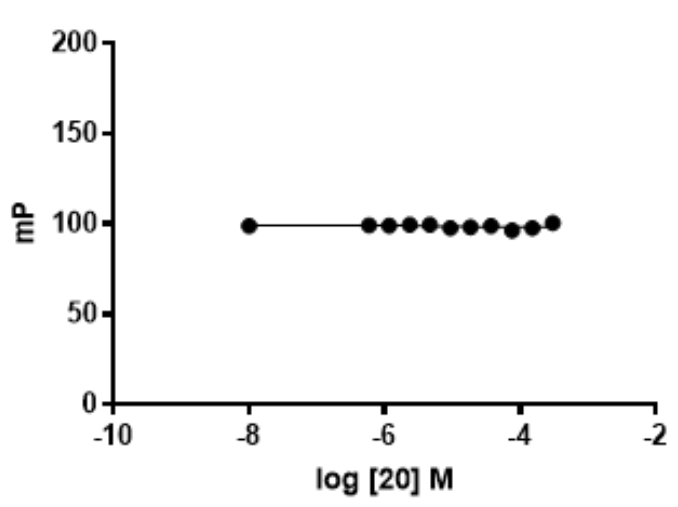

C)

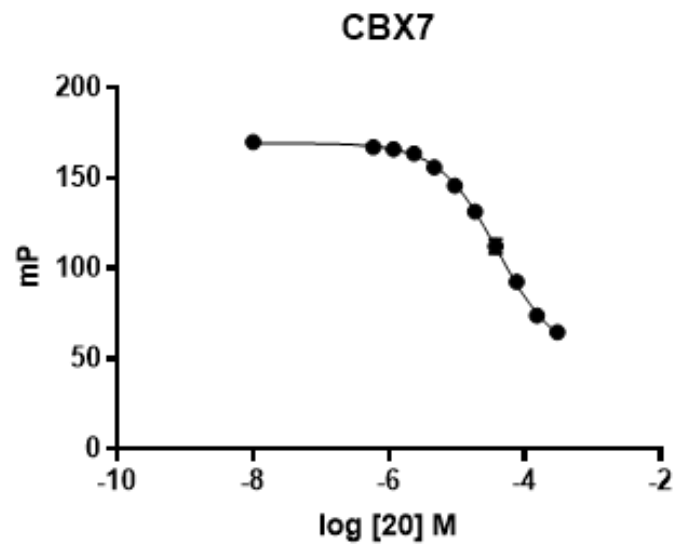

B)

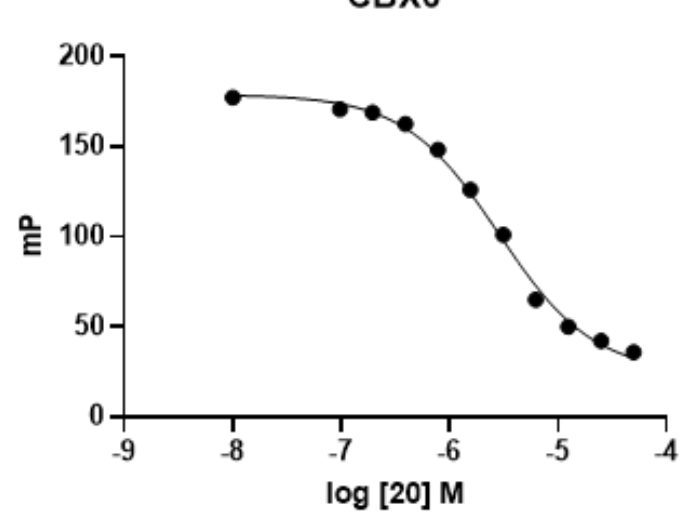

D)

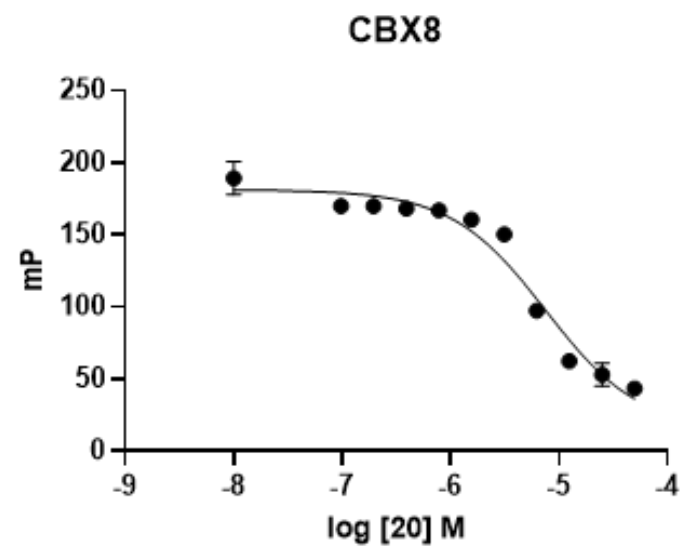

Supplementary Figure S40: Competitive fluorescence polarization data of compound 20 with CBX proteins performed in triplicate. A) $\left.\mathrm{CBX} 1\left(\log \mathrm{IC}_{50}=>-3.301 \mathrm{M}\right) \mathrm{B}\right) \mathrm{CBX} 6\left(\log \mathrm{IC}_{50}=-\right.$ $5.546 \mathrm{M}, 95 \% \mathrm{CI}-5.592 \mathrm{M}$ to $\left.\left.-5.499 \mathrm{M}, \mathrm{R}^{2}=0.996\right), \mathrm{C}\right) \mathrm{CBX} 7\left(\log \mathrm{IC}_{50}=-4.401 \mathrm{M}, 95 \% \mathrm{CI}-\right.$ $4.438 \mathrm{M}$ to $\left.-4.364 \mathrm{M}, \mathrm{R}^{2}=0.998\right)$, D) $\mathrm{CBX} 8\left(\log \mathrm{IC}_{50}=-5.125 \mathrm{M}, 95 \% \mathrm{CI}-4.989 \mathrm{M}\right.$ to -5.301 $\left.\mathrm{M}, \mathrm{R}^{2}=0.964\right)$. Error bars reported as asymmetrical $95 \%$ confidence intervals. 
A)

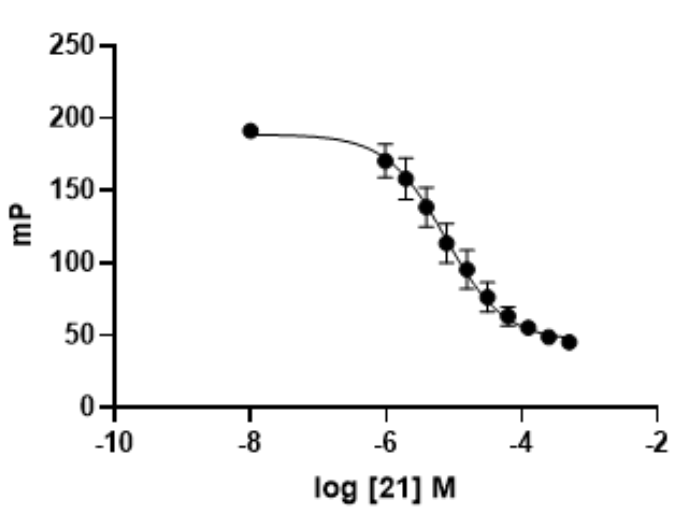

C)

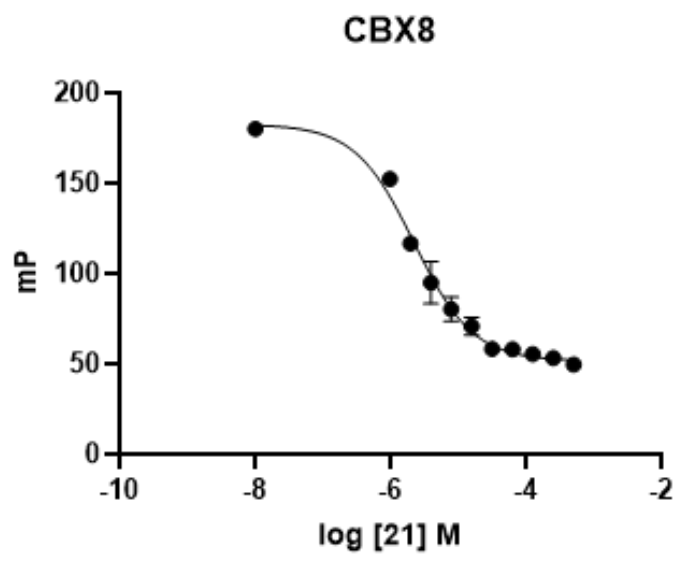

B)

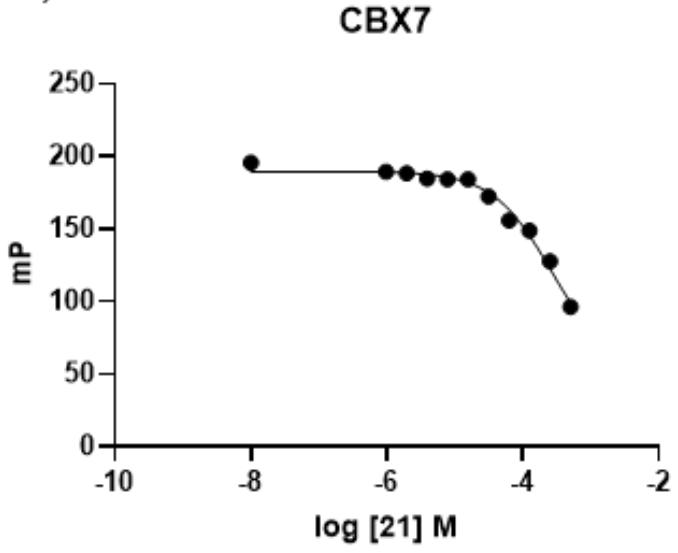

Supplementary Figure S41: Competitive fluorescence polarization data of compound 21 with CBX proteins performed in triplicate. A) CBX6 $\left(\log \mathrm{IC}_{50}=-5.126 \mathrm{M}, 95 \% \mathrm{CI}-5.244 \mathrm{M}\right.$ to $\left.\left.5.006 \mathrm{M}, \mathrm{R}^{2}=0.973\right), \mathrm{B}\right) \mathrm{CBX} 7\left(\log \mathrm{CC}_{50}=-3.572 \mathrm{M}, 95 \% \mathrm{CI}-3.721 \mathrm{M}\right.$ to $-3.406 \mathrm{M}, \mathrm{R}^{2}=$ $0.979), \mathrm{C}) \mathrm{CBX} 8\left(\log \mathrm{IC}_{50}=-5.656,95 \% \mathrm{CI}-5.732 \mathrm{M}\right.$ to $\left.-5.578 \mathrm{M}, \mathrm{R}^{2}=0.984\right)$. Error bars reported as asymmetrical $95 \%$ confidence intervals. 
A)

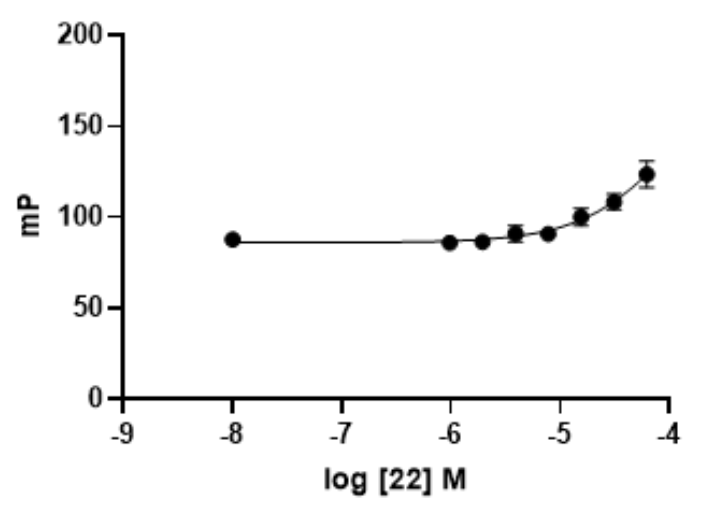

C)

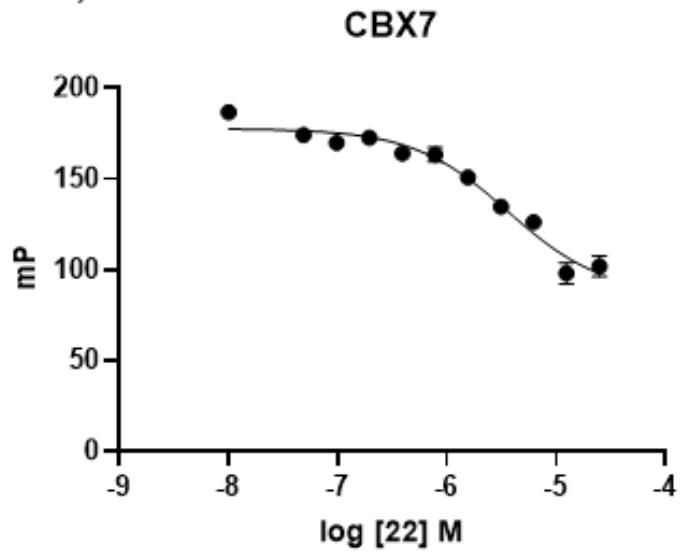

B)

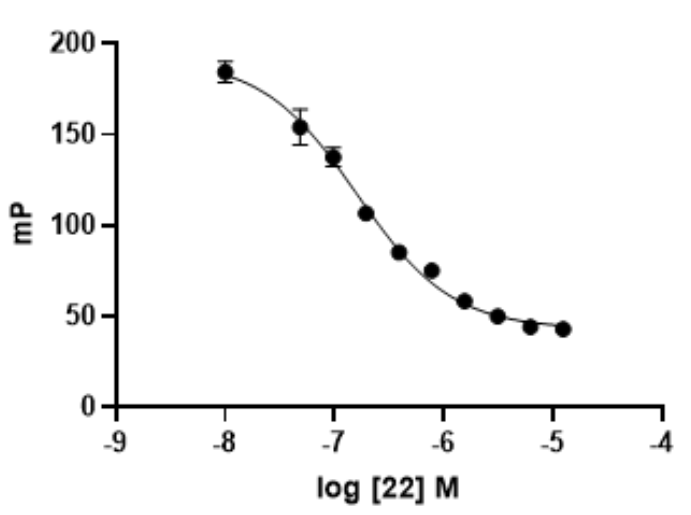

D)

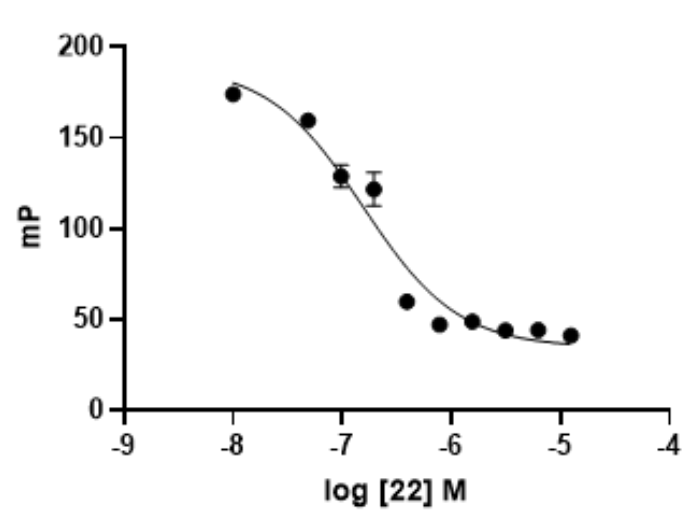

Supplementary Figure S42: Competitive fluorescence polarization data of compound 22 with CBX proteins performed in triplicate. A) CBX1 $\left.\left(\log \mathrm{IC}_{50}=>-3.301 \mathrm{M}\right) \mathrm{B}\right) \mathrm{CBX} 6\left(\log \mathrm{IC}_{50}=-\right.$ $6.793 \mathrm{M}, 95 \% \mathrm{CI}-6.866 \mathrm{M}$ to $\left.\left.-6.719 \mathrm{M}, \mathrm{R}^{2}=0.990\right), \mathrm{C}\right) \mathrm{CBX} 7\left(\log \mathrm{IC}_{50}=-5.440 \mathrm{M}, 95 \% \mathrm{CI}-\right.$ $5.600 \mathrm{M}$ to $\left.\left.-5.278 \mathrm{M}, \mathrm{R}^{2}=0.960\right), \mathrm{D}\right) \mathrm{CBX} 8\left(\log \mathrm{IC}_{50}=-6.809 \mathrm{M}, 95 \% \mathrm{CI}-6.945 \mathrm{M}\right.$ to -6.672 $\left.\mathrm{M}, \mathrm{R}^{2}=0.957\right)$. Error bars reported as asymmetrical $95 \%$ confidence intervals. 

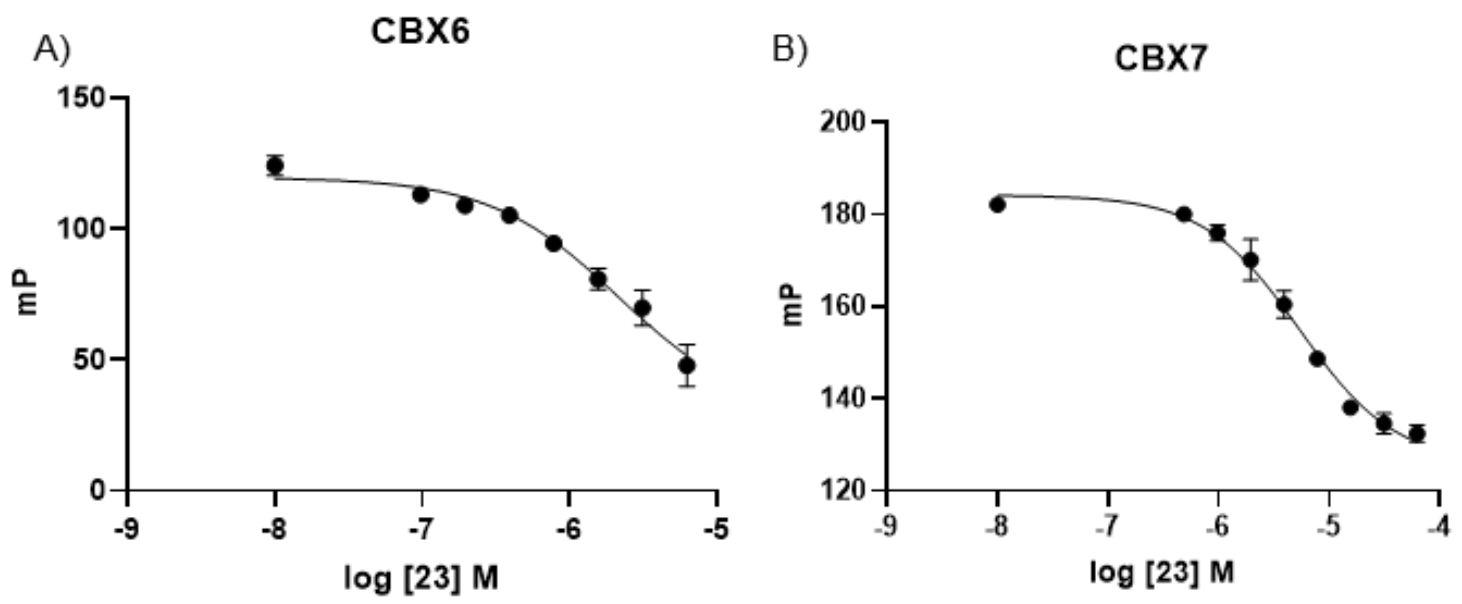

C)

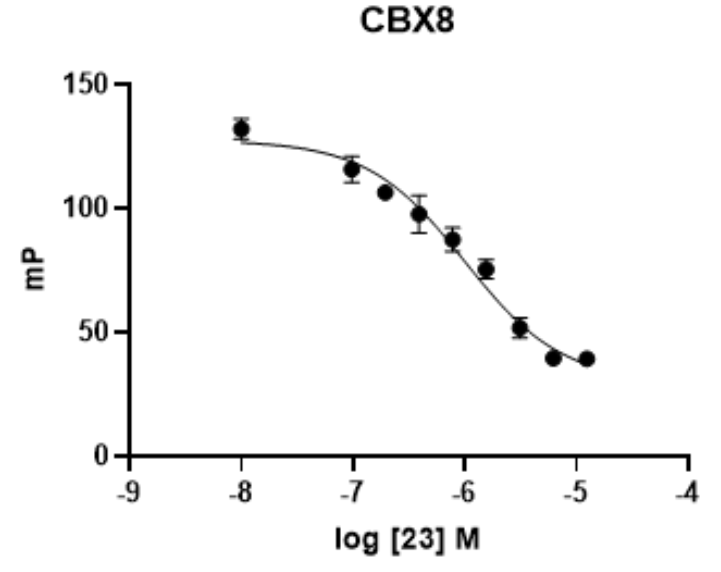

Supplementary Figure S43: Competitive fluorescence polarization data of compound 23 with CBX proteins performed in triplicate. A) CBX6 $(\operatorname{logIC} 50=-5.667 \mathrm{M}, 95 \% \mathrm{CI}-5.895 \mathrm{M}$ to $\left.\left.5.412 \mathrm{M}, \mathrm{R}^{2}=0.961\right), \mathrm{B}\right) \mathrm{CBX} 7\left(\log \mathrm{IC} 50=-5.270 \mathrm{M}, 95 \% \mathrm{CI}-5.360 \mathrm{M}\right.$ to $-5.177 \mathrm{M}, \mathrm{R}^{2}=$ $0.985)), \mathrm{C}) \mathrm{CBX} 8\left(\log \mathrm{IC} 50=-5.999,95 \% \mathrm{CI}-6.147 \mathrm{M}\right.$ to $\left.-5.849 \mathrm{M}, \mathrm{R}^{2}=0.975\right)$. Error bars reported as asymmetrical $95 \%$ confidence intervals. 
A)

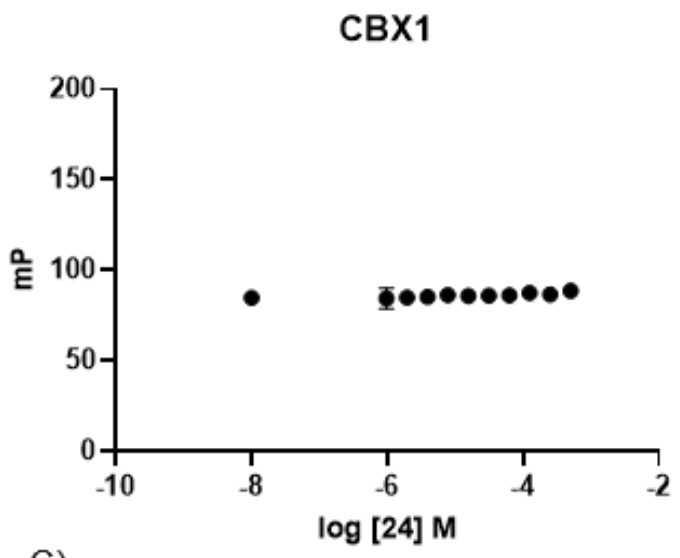

C)

CBX7

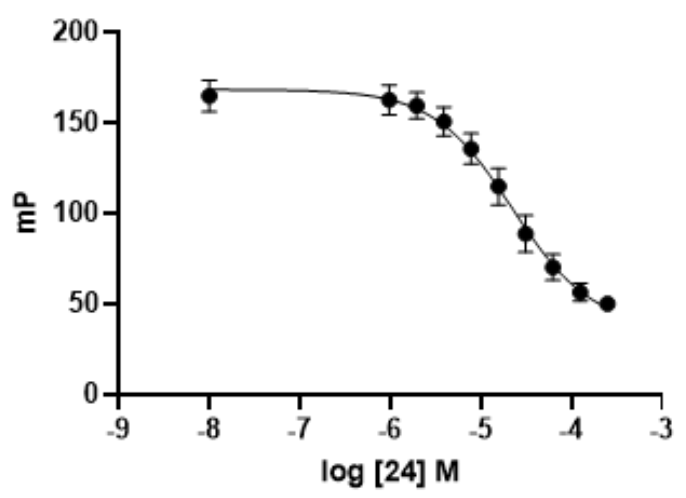

B)

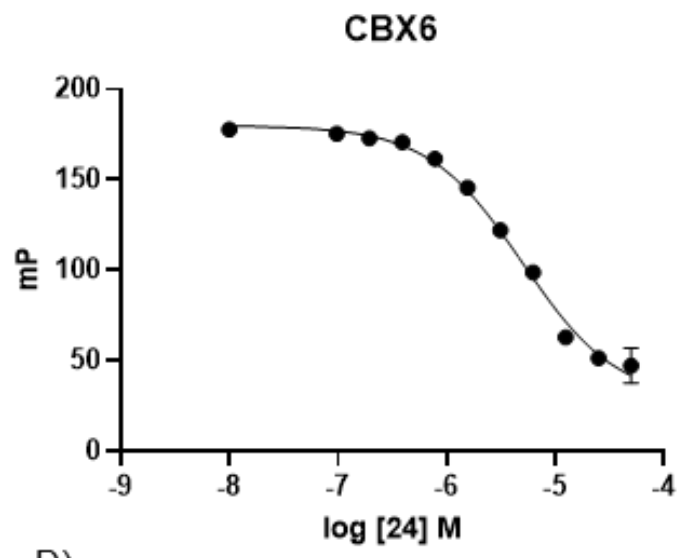

D)

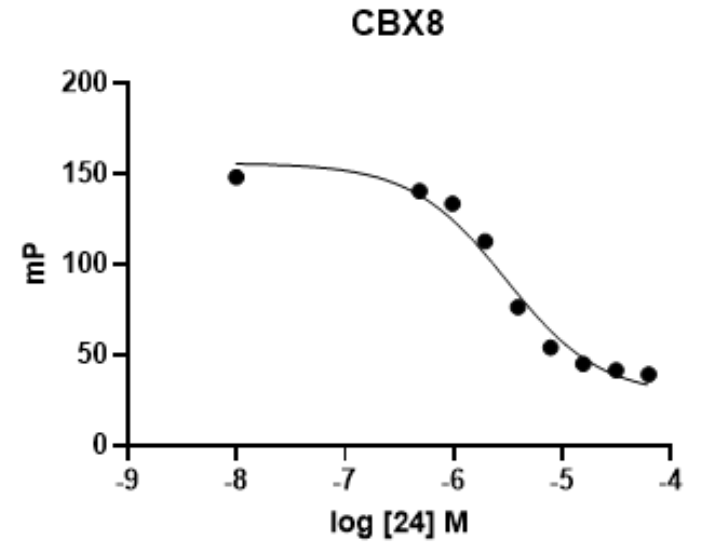

Supplementary Figure S44: Competitive fluorescence polarization data of compound 24 with CBX proteins performed in triplicate. A) $\left.\mathrm{CBX} 1\left(\operatorname{logIC} \mathrm{C}_{50}=>-3.301 \mathrm{M}\right) \mathrm{B}\right) \mathrm{CBX} 6\left(\log \mathrm{CC}_{50}=-\right.$ $5.300 \mathrm{M}, 95 \% \mathrm{CI}-5.362 \mathrm{M}$ to $\left.\left.-5.237 \mathrm{M}, \mathrm{R}^{2}=0.993\right), \mathrm{C}\right) \mathrm{CBX} 7\left(\log \mathrm{IC}_{50}=-4.654 \mathrm{M}, 95 \% \mathrm{CI}-\right.$ $4.768 \mathrm{M}$ to $\left.-4.538 \mathrm{M}, \mathrm{R}^{2}=0.977\right)$, D) $\mathrm{CBX} 8\left(\log \mathrm{IC}_{50}=-5.518 \mathrm{M}, 95 \% \mathrm{CI}-5.620 \mathrm{M}\right.$ to -5.414 $\left.\mathrm{M}, \mathrm{R}^{2}=0.976\right)$. Error bars reported as asymmetrical $95 \%$ confidence intervals. 
A)

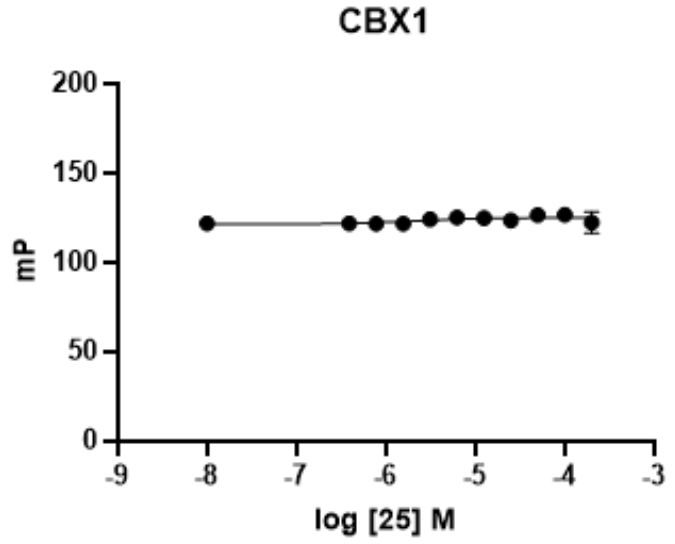

C)

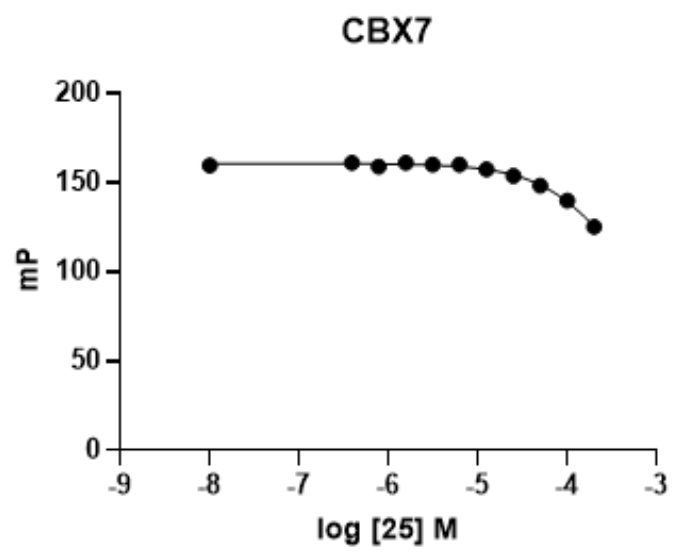

B)

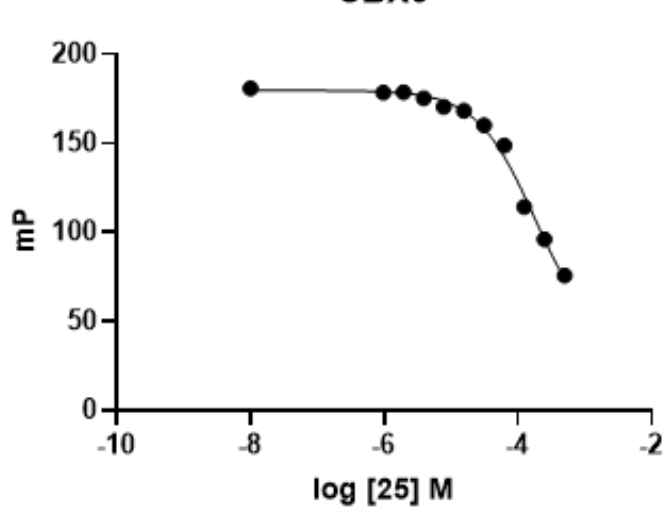

D)

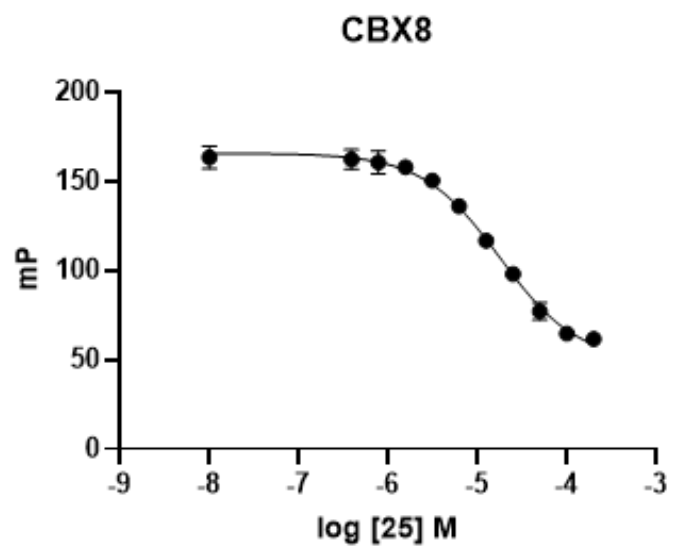

Supplementary Figure S45: Competitive fluorescence polarization data of compound 25 with CBX proteins performed in triplicate. A) CBX1 $\left.\left(\log \mathrm{IC}_{50}=>-3.699 \mathrm{M}\right) \mathrm{B}\right) \mathrm{CBX} 6\left(\operatorname{logIC} \mathrm{C}_{50}=-\right.$ $3.750 \mathrm{M}, 95 \% \mathrm{CI}-3.837 \mathrm{M}$ to $\left.\left.-3.659 \mathrm{M}, \mathrm{R}^{2}=0.989\right), \mathrm{C}\right) \mathrm{CBX} 7\left(\operatorname{logIC} \mathrm{C}_{50}=-3.43 \mathrm{M}, 95 \% \mathrm{CI}-\right.$ $3.458 \mathrm{M}$ to $\left.-3.400 \mathrm{M}, \mathrm{R}^{2}=0.983\right)$, D) $\mathrm{CBX} 8\left(\log \mathrm{IC}_{50}=-4.751 \mathrm{M}, 95 \% \mathrm{CI}-4.824 \mathrm{M}\right.$ to -4.678 $\left.\mathrm{M}, \mathrm{R}^{2}=0.989\right)$. Error bars reported as asymmetrical $95 \%$ confidence intervals. 
A)

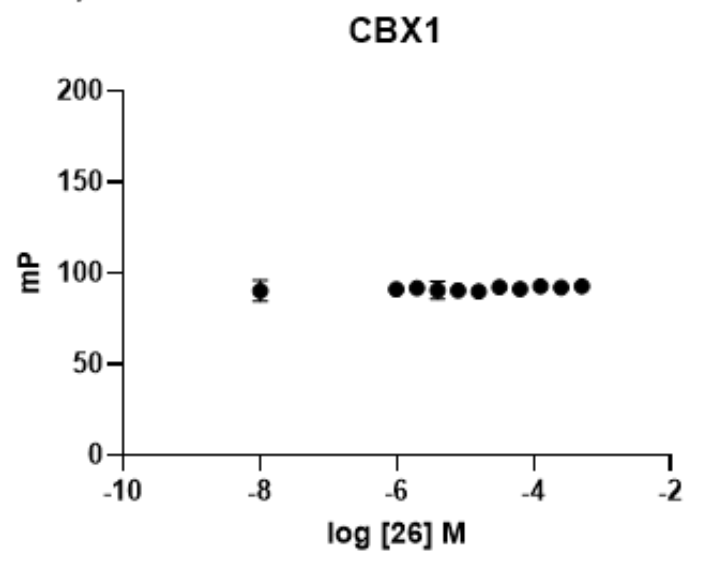

C)

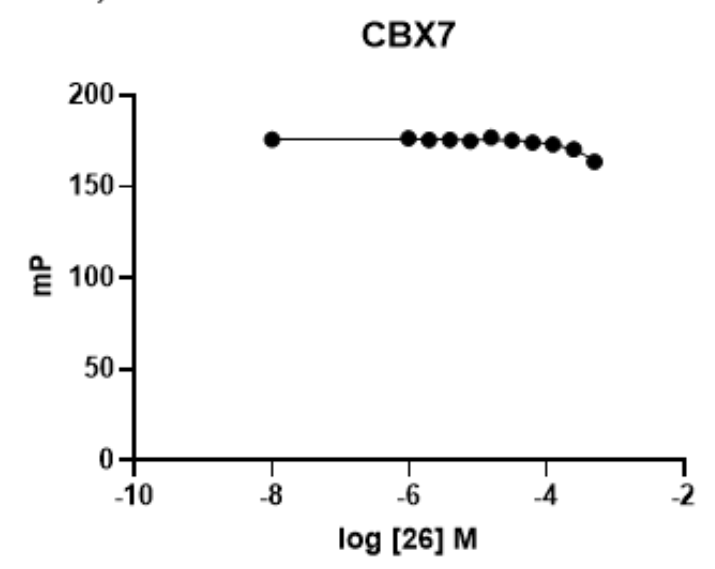

B)

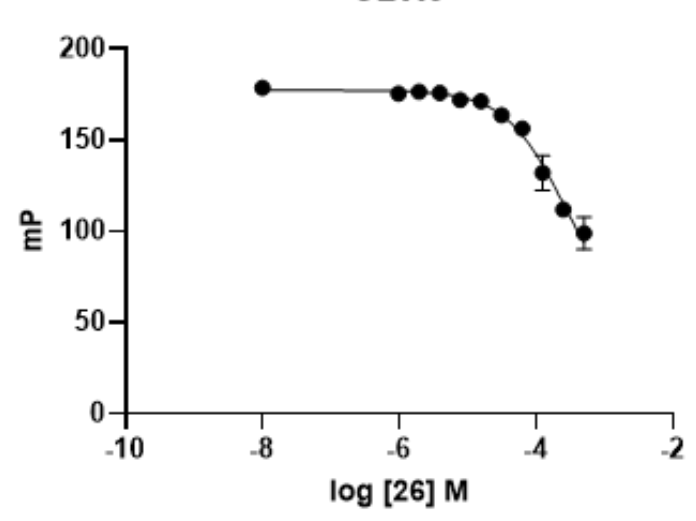

D)

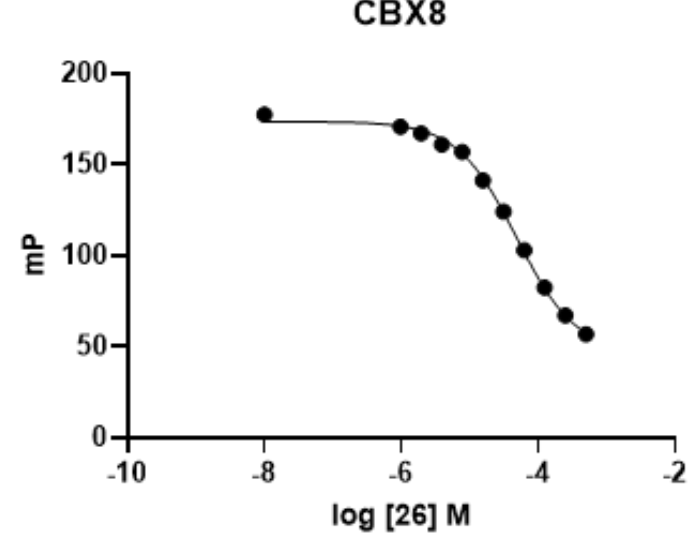

Supplementary Figure S46: Competitive fluorescence polarization data of compound 26 with CBX proteins performed in triplicate. A) CBX1 $\left.\left(\operatorname{logIC} \mathrm{C}_{50}=>-3.301 \mathrm{M}\right), \mathrm{B}\right) \mathrm{CBX} 6\left(\log \mathrm{CC}_{50}=-\right.$ $3.582 \mathrm{M}, 95 \% \mathrm{CI}-3.631 \mathrm{M}$ to $\left.-3.522 \mathrm{M}, \mathrm{R}^{2}=0.973\right)$, C) $\mathrm{CBX} 7\left(\log \mathrm{IC}_{50}=>-3.301 \mathrm{M}\right)$, D) CBX8 $\left(\log \mathrm{IC}_{50}=-4.315 \mathrm{M}, 95 \% \mathrm{CI}-4.356 \mathrm{M}\right.$ to $\left.-4.274 \mathrm{M}, \mathrm{R}^{2}=0.997\right)$. Error bars reported as asymmetrical $95 \%$ confidence intervals. 


\section{Methods for cellular Assays}

\section{Generation of CBX6 KO cell lines:}

Oligonucleotides for control guide RNA (sgControl: 5'-GTAGCGAACGTGTCCGGCGT-3') and CBX6 targeting guide RNAs (sgCBX6_1: 5'-CATCAAACGGCGGATCCGAA-3' ; sgCBX6_2: 5'-TCGGATCCGCCGTTTGATGA-3') were cloned into lenticrispr v2.0 vector (a gift from Feng Zhang Addgene plasmid no. 52961). HEK293T cells were used to make lentiviral particles for each construct. G401 cells were transduced with concentrated virus particles and selected with puromycin to generate stable cell lines.

\section{CellTiter-Glo luminescent cell viability assay}

The effect of $\mathbf{8}, \mathbf{1 1}, \mathbf{2 2}$ and $\mathbf{2 3}$ on cell viability was determined using a CellTiter-Glo ATP detection system (\#G7573, Promega). G401 cells were seeded in $0.1 \times 10^{\wedge} 6$ cells $/ \mathrm{mL}$ density in 96-well clear bottom white microplate (\#655098, Greiner Bio-One). Peptides (50, $100 \mu \mathrm{M})$ were added to the cells with $0.5 \%$ DMSO. After $3 \mathrm{~d}$, CellTiter-Glo reagent was added to cells, and incubated with gentle shake for 15 minutes in dim light at R.T. Luminescence was read on an GloMax ${ }^{\circledR}$ microplate reader. 

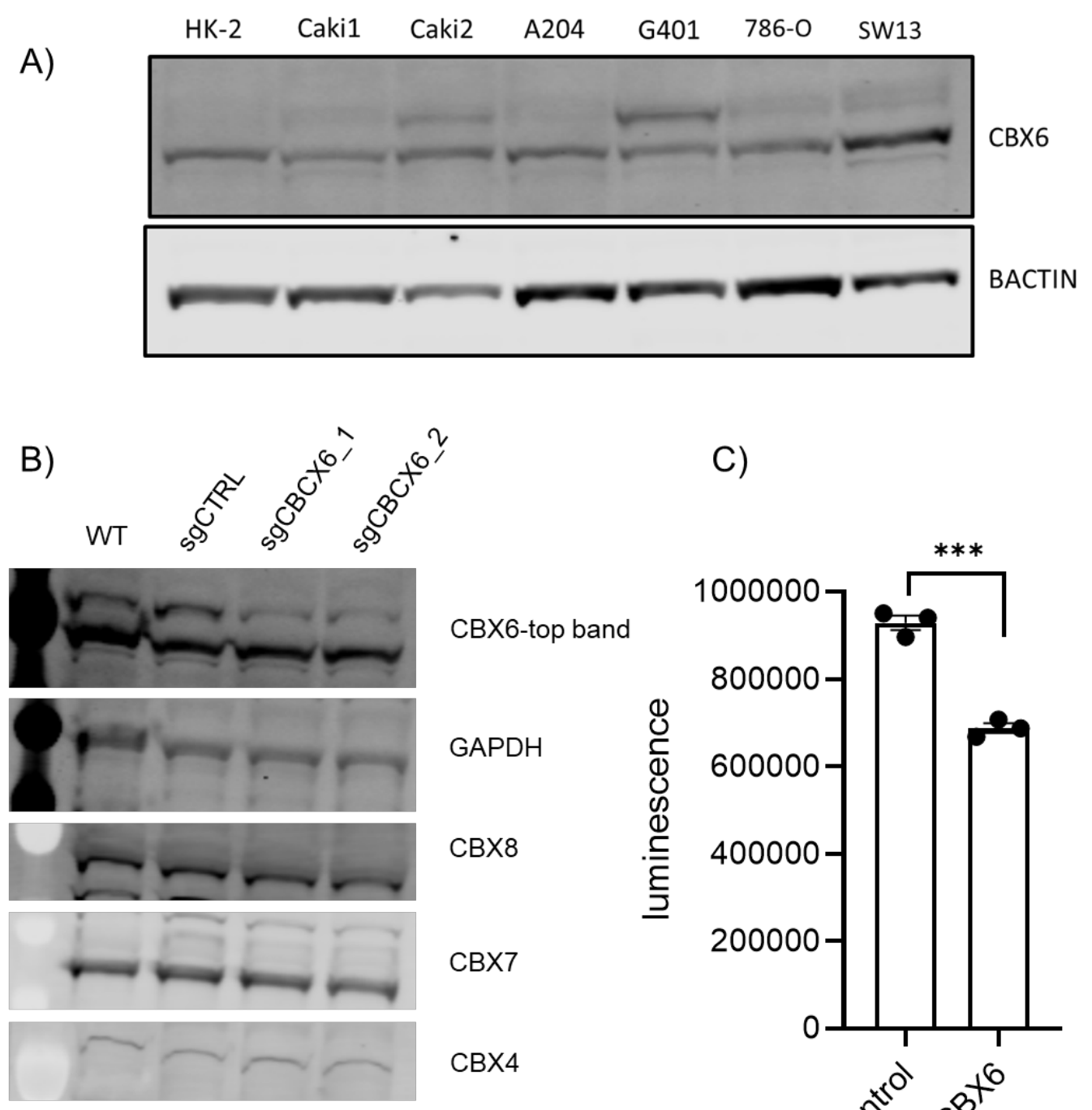

CBX7

C)

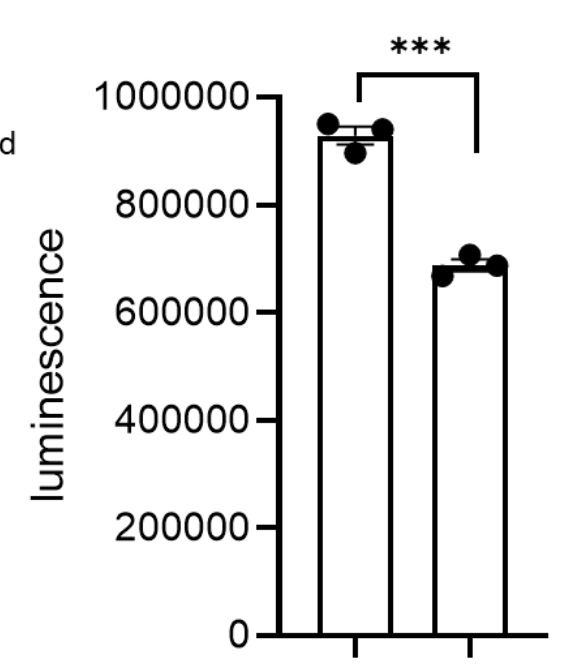

CBX4

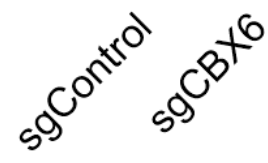

Supplementary Figure S47: Generation of CBX6 knockout in G401 Rhabdoid tumor cell line. A) Immunoblot analysis of whole cell lysates from kidney epithelial cells (HK2), clear cell renal cancer cell lines (Caki1, Caki2, 786O), kidney adrenal cancer (SW13), kidney rhabdoid tumor (G401, and muscle rhabdoid tumor A204. Top band is CBX6 and bottom band is non-specific. Beta actin was included as a loading control. B) CRISPR-Cas9 was used to knock out CBX6 in G401 cells. Immunoblot analysis confirms a reduction in CBX6, but not other CBX subunit protein levels. C) G401 cells were grown for $72 \mathrm{~h}$ and luminescence was measured after addition of CellTiter Glo®. 